\title{
Exploring the Role of Prospective Memory in Location-Based Reminders
}

\author{
Yao Wang \\ Dissertation submitted to the Faculty of the \\ Virginia Polytechnic Institute and State University \\ in partial fulfillment of the requirements for the degree of
}

Doctor of Philosophy

in

Computer Science and Applications

Manuel A. Pérez-Quiñones, Chairman
Scott McCrickard
Eli Tilevich
Rachel Diana
Mark Schlager

April 19, 2017

Blacksburg, Virginia

Keywords: ubiquitous computing, prospective memory, location-based reminder, external memory aids

Copyright 2017, Yao Wang 


\title{
Exploring the Role of Prospective Memory in Location-Based Reminders Yao Wang
}

\begin{abstract}
Location-based reminder systems (LBRs) are typically used to remind people to complete a to-do task at a particular location. People use their prospective memory to remember future to-do tasks. However, the current design of LBRs fails to take advantage of human prospective memory theory. In this dissertation, I propose a framework connecting human prospective memory theory with LBRs. My work applies human prospective memory into the technical design of LBRs. The goal of my work is to make the reminder work more consistently with how human memory works.
\end{abstract}

Prospective memory research suggests that encoding of the location and familiarity with the location have an impact on prospective remembering. I conducted two empirical studies to test how this theoretical knowledge applies to LBRs. In one experiment, I hypothesized that if the encoding stage provides a closer match to the retrieval stage in LBRs, then location recognition and task recall should improve at retrieval time. The results indicate that providing a first-person view (street view of the desired location) at the encoding stage benefits prospective remembering the most.

Prospective memory theory also suggests that the familiarity with the external cue has a significant influence on prospective remembering. In the second experiment, I hypothesized that familiarity with a location has an impact on the location recognition at the retrieval. The results show that the encoding interface is used differently for familiar and unfamiliar cities and businesses to support recognizing a target location.

The findings have implications for the design of future LBRs. I designed an LBR prototype by applying these empirical research findings and conducted a usability evaluation. Future 
designers of LBR should consider 1) providing more support in matching the encoding stage to the eventual cue in retrieval stage and 2) involving user's familiarity level with the places at the encoding stage to provide a better user experience. My work showed the importance of using prospective memory theory in the design of LBR systems. 


\section{Exploring the Role of Prospective Memory in Location-Based Reminders Yao Wang}

\section{General Audience Abstract}

People use location-based mobile reminder systems to complete a to-do task at a particular location. My work studied how people memorize the locations at the reminder set up stage, as well as how they recognize the locations and remember the associated to-do tasks at the reminder retrieval stage. People see a location from a first-person view while completing a to-do task in the real life. However, current interface designs of location-based reminders do not support that. My research results contributed to the future interface design of location-based mobile reminder system. That is, future encoding interface design of location-based reminders could possibly apply a street view of the location to make the reminder more usable and more user-friendly. 


\section{Dedication}

Dedicated to my husband $B o L i$, who is always there with me.

Dedicated to my baby boy Chengyu $L i$, who gives me courage to meet the challenge.

Dedicated to my parents, Lianzeng Wang and Hui Yang, for their support and love. 


\section{Acknowledgments}

This dissertation can not be completed without a lot of support from my family, my academic advisors and my friends. First and foremost, I would like to give many thanks to my advisor Dr. Manuel A. Pérez-Quiñones. He is always supporting and encouraging me during my Ph.D. study. He helped me grow, survive and finally reach this milestone. I would like to thank Dr. Rachael Diana, who gave me a lot of help and support when I got lost in my research. I am very thankful to Dr. Mark Schlager for his insightful advice. He helped me a lot polish my initial research ideas and taught me how to conduct research. I would also like to thank my committee members Dr. Eli Tilevich and Dr. Scott McCrickard for the guidance and comments.

I have been very fortunate to work with some great friends while studying at Virginia Tech. I am very thankful to my wonderful lab-mates, Michael Stewart, Robert Beaton, Nai-Ching Wang, Sheriff Jolaoso, Will Ellis and Ben Hanrahan. I would like to give many thanks to Min Li and Yue Cheng, who helped me a lot in doing my research. I have also been very lucky to know nice friends when I was in Blacksburg, Anamary Leal, Fatma Elzahraa Eid, Mai El Shehaly, Qianzhou Du, Fang Liu, Hao Zhang, Maoyuan Sun, Ji Wang, Zheng Song, Tianzi Wang, Jingyi Yin, Yue Sun, and Yujie Cheng.

I would like to thank the center of Human-Computer Interaction for providing me a great environment to do research. I would also like to thank the Association of Women in Com- 
puting for giving me great opportunities to attend conferences and make connections, as well as lots of support for my life. 


\section{Contents}

1 Introduction $\quad 1$

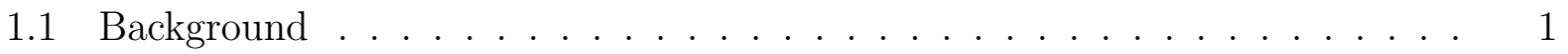

1.2 Motivation . . . . . . . . . . . . . . . . . . . . . 3

1.2.1 Unreliable Technology . . . . . . . . . . . . . . . . 3

1.2.2 Cognitive Offloading ......................... 4

1.3 Research Problem . . . . . . . . . . . . . . . . . . . . 5

1.4 Research Questions and Hypotheses . . . . . . . . . . . . . . . . . . 8

1.4.1 RQ 1 - Matching Encoding and Retrieval . . . . . . . . . . . 8

1.4.2 RQ 2 - The Impact of Familiarity . . . . . . . . . . . . . . . . . . 9

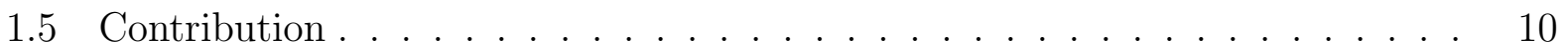

1.6 Overview of the Dissertation . . . . . . . . . . . . . . . 11

2 Theoretical Foundation $\quad 13$

2.1 Prospective Memory . . . . . . . . . . . . . . . . . . 14 
2.1 .1 Prospective Memory Tasks . . . . . . . . . . . . . . . . . . . . . . 14

2.1.2 External Cues . . . . . . . . . . . . . . . . . . . . . . . . 15

2.1 .3 Encoding and Retrieval . . . . . . . . . . . . . . . . . 16

2.2 Prospective Remembering $\ldots \ldots \ldots \ldots \ldots$

2.2 .1 Cognitive Retrieval Process _. . . . . . . . . . . . . . . 17

2.2 .2 Implementation Intention $\ldots \ldots \ldots \ldots \ldots \ldots$

2.2.3 Familiarity of External Cues in Prospective Remembering . . . . . . 18

2.3 Prospective Forgetting $\ldots \ldots \ldots \ldots$

2.3 .1 Poor Encoding $\ldots \ldots \ldots \ldots$

$2.3 .2 \quad$ Cues Fail . . . . . . . . . . . . . . . . . . . . . . . . . . . 20

2.4 External Memory Aids . . . . . . . . . . . . . . . . . . . . . . 21

2.5 Spatial Knowledge . . . . . . . . . . . . . . . . . . . . . . . . . . 22

2.5.1 Types of Spatial Knowledge . . . . . . . . . . . . . . . . 23

2.5.2 Familiarity of locations in Spatial Knowledge . . . . . . . . . . 24

2.5 .3 Naive Geography . . . . . . . . . . . . . . . . . . . . . 25

2.5.4 Hierarchical Structure of Location . . . . . . . . . . . . . . 25

2.6 Theoretical Foundation for User Study $1 \ldots \ldots 26$

2.7 Theoretical Foundation for User Study $2 \ldots \ldots$. . . . . . . . . . 27

3 Prior Work in Reminders $\quad 29$ 
3.1 Human Memory Aids . . . . . . . . . . . . . . . . . . . . . 29

3.2 Reminder Applications . . . . . . . . . . . . . . . . . . . 30

3.3 Location-based Reminders (LBRs) . . . . . . . . . . . . . . . 32

3.3.1 Early Design of LBRs . . . . . . . . . . . . . . . 32

3.3.2 LBRs to Remind Daily To-do Tasks . . . . . . . . . . . . . . . . 33

3.3.3 LBRs to Support Social Life . . . . . . . . . . . . . . . . 34

3.3.4 Delivery mechanism of LBRs . . . . . . . . . . . . . . . 35

3.3.5 User Evaluations of LBRs . . . . . . . . . . . . . . . . 37

3.3.6 State-of-the-art LBRs . . . . . . . . . . . . . . . . 38

4 Usability Study of Existing LBRs $\quad 42$

4.1 User Study Setup . . . . . . . . . . . . . . . . . . . . . 42

4.2 Study Procedure . . . . . . . . . . . . . . . . . . 43

4.3 Usability Issues . . . . . . . . . . . . . . . . . . . . . 44

4.4 Considering Human Spatial Cognition . . . . . . . . . . . . . . . 46

4.5 Summary . . . . . . . . . . . . . . . . . . . . 47

5 Classification of Location-based To-do Tasks 48

5.1 Online Survey . . . . . . . . . . . . . . . . . . . . . 48

5.2 Data Analysis . . . . . . . . . . . . . . . . . . . . . 49

5.3 Classification of Uses of Locations . . . . . . . . . . . . . . . . 50 
5.3 .1 Do Task X at Location A . . . . . . . . . . . . . . 51

5.3 .2 Deliver X from A to B . . . . . . . . . . . . . . 51

5.3 .3 Go to Location A . . . . . . . . . . . . . . . . . . . . . . 52

5.4 Summary . . . . . . . . . . . . . . . . . . . . . 53

6 Framework $\quad 54$

7 The Match Between Encoding and Retrieval 58

$7.1 \quad$ Experimental Design . . . . . . . . . . . . . . . . . . . . 58

7.2 Study Setup . . . . . . . . . . . . . . . . . . . . . 6 61

$7.2 .1 \quad$ Participants . . . . . . . . . . . . . . . . . . . 61

$7.2 .2 \quad$ Procedure . . . . . . . . . . . . . . . . . . . . . . . . 62

7.2 .3 Data Collection . . . . . . . . . . . . . . . . . . . . 66

7.3 Results . . . . . . . . . . . . . . . . . . . . 67

7.3 .1 Complete Recall . . . . . . . . . . . . . . . . . . . . . . . . . 67

7.3 .2 Location Recognition . . . . . . . . . . . . . . . . . . . . . . . . 69

7.3 .3 Task Recall . . . . . . . . . . . . . . . . . . . . . . . . . . . 70

7.3 .4 Encoding Effort . . . . . . . . . . . . . . . . . . . . . . . 71

7.3 .5 Retrieval Effort . . . . . . . . . . . . . . . . . . . . . 71

7.3 .6 The Impact of Familiarity . . . . . . . . . . . . . . . . . . . . 72

7.3 .7 Task Frequency and Task Recall . . . . . . . . . . . . . . . . . . 77 
7.3.8 Gender and Memory . . . . . . . . . . . . . . 77

7.3 .9 Familiarity Level . . . . . . . . . . . . . . . . 79

7.3 .10 Familiarity with Daily Errands . . . . . . . . . . . . . 79

7.4 Design Implications . . . . . . . . . . . . . . . . . . . . . . 80

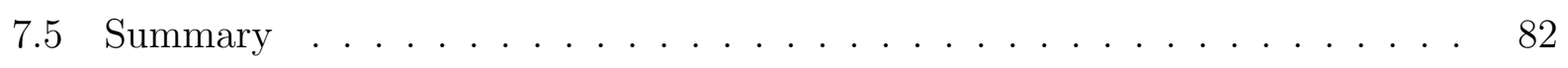

8 The Impact of Familiarity $\quad 83$

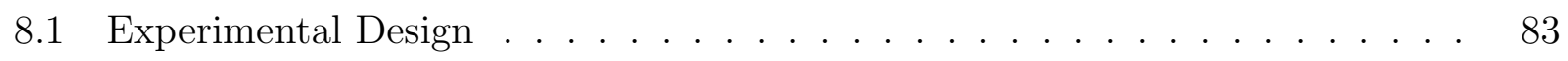

8.2 Study Setup . . . . . . . . . . . . . . . . . . 87

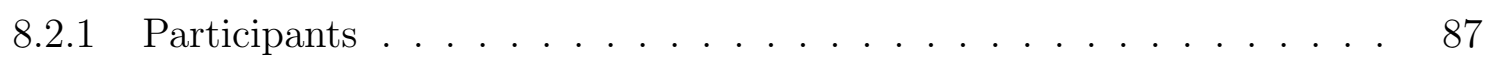

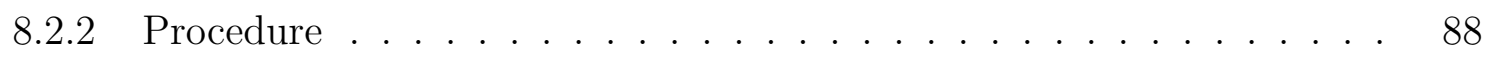

8.2 .3 Data Collection . . . . . . . . . . . . . . . 90

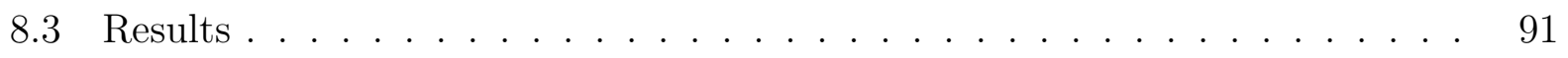

8.3.1 Familiar City (home) . . . . . . . . . . . . . 91

8.3.2 Unfamiliar City (traveling) . . . . . . . . . . . . . . . 92

8.3.3 Familiarity of Places in a Familiar City . . . . . . . . . . . . . . 94

8.3.4 Familiarity of Places in an Unfamiliar City . . . . . . . . . . . . 98

8.3.5 Interactive Street View . . . . . . . . . . . . . . . . . 102

8.3.6 Topographical Features of the City . . . . . . . . . . . 106

8.4 Design Implications . . . . . . . . . . . . . . . . . . . 106 
8.4.1 Implications for LBRs "At Home" . . . . . . . . . . . . . . . . 106

8.4.2 Implications for LBRs "While Traveling" . . . . . . . . . . . . . . 107

8.4.3 Familiarity with City More Impactful Than Place . . . . . . . . . . 107

8.4.4 Adaptation of interface according to familiarity . . . . . . . . . 108

8.5 Summary . . . . . . . . . . . . . . . . . . . . 109

9 LBR Prototype Design and Evaluation $\quad 110$

9.1 Introduction . . . . . . . . . . . . . . . . 110

9.2 Iterative Design of Prototype $\ldots \ldots \ldots \ldots \ldots \ldots$

9.2.1 Setup Reminder at Generic Location . . . . . . . . . . . . . . . 112

9.2.2 Setup Reminder at Particular Location . . . . . . . . . . . . . 120

9.3 Usability Evaluation $\ldots \ldots \ldots \ldots \ldots$

9.3 .1 Participants . . . . . . . . . . . . . . . . . . . 120

9.3 .2 Data Collection . . . . . . . . . . . . . . . . . . 125

9.3 .3 Task Scenarios . . . . . . . . . . . . . . . . . . . . . . . . . . . 125

9.4 Results . . . . . . . . . . . . . . . . . . . . . 126

9.4 .1 Subjective Comments . . . . . . . . . . . . . . . . . 126

9.4 .2 Usability Issues . . . . . . . . . . . . . . . . . . . . . . . . 127

9.5 Design Implications . . . . . . . . . . . . . . . . . . . . 128

9.6 Summary . . . . . . . . . . . . . . . . . . . . . . . 130 
10 Conclusions and Future Work

10.1 Conclusions . . . . . . . . . . . . . . . . . . . . 131

10.2 Future Work . . . . . . . . . . . . . . . . . . . . . . . 132

10.2.1 Route Planner With LBR . . . . . . . . . . . . . . . . 133

10.2.2 Context-aware Reminder . . . . . . . . . . . . . . . . 133

10.2.3 What Information to Remind . . . . . . . . . . . . . . . 134

Bibliography

A Survey Materials for Chapter 5

B User Study Materials for Chapter 7

C User Study Materials for Chapter 8

D Prototype Evaluation Materials for Chapter 9 


\section{Chapter 1}

\section{Introduction}

Location-based reminders are reminder messages that can be triggered based on a person's physical location. With the proliferation of position sensing technology (e.g., GPS) on mobile devices, location-based reminders (LBRs) have been widely developed. However, sometimes LBRs fail to deliver the reminders appropriately. Researchers have conducted studies in the fields of context-awareness and ubiquitous computing to enhance the effectiveness of location-based reminder delivery, but none of the studies has specifically investigated the role of prospective memory in LBRs. The focus of my research is on understanding the theoretical knowledge of human prospective memory, and in applying the psychological research findings to the design of LBRs. The goal is to make LBRs work more consistently with how human memory works and to facilitate how people remember and recall location-based to-do tasks.

\subsection{Background}

Human memory is fallible; people sometimes forget things. When people need to remember to perform an intended task in the future, it involves human prospective memory 
[Einstein and McDaniel, 1990]. For example, a typical prospective task is to remember to stop at a grocery store to buy milk after work while on the way home. Forgetting to do things is a common type of memory failure in a person's daily life. Schacter [Schacter, 1999] defined prospective memory failure as a type of absentmindedness, which occurs when insufficient attention is devoted at the encoding or retrieval stage. Moreover, remembering to perform a to-do task requires that people remember to interrupt the current flow of thoughts or activities, and switch attention to the intended to-do task, so it makes prospective remembering harder. For example, people easily forget to stop at the gas station when the cognitive demand of driving is high.

Forgetting to-do things can sometimes get people in trouble, so people use memory aids to help them remember. Forgetting an appointment might make someone upset, and forgetting to take medicine on time can put a person in a dangerous condition. To help someone remember to-do tasks, memory aids have been used and studied [Harris, 1978].

Today, people use various kinds of reminders to support them remembering to-do tasks. Intone-Peterson and Fournier [Intons-Peterson and Fournier, 1986] proved that external memory aids were generally used in supporting prospective memory. External memory aids that particularly help people remember future to-do things are known as reminders. Knowledge workers constantly keep track of many things to do in the office, while working outside of the office, or even at home. To manage work, they often make use of calendar alerts, to-do lists and Post-it notes as reminders. Location-based mobile reminders (LBRs) are particularly used to remind people to complete a task at a particular location by sending them alerts when they are near said location. For example, a person driving by a particular dry cleaner might get a reminder to pick up their laundry. 


\subsection{Motivation}

\subsubsection{Unreliable Technology}

Prior work with LBRs has shown that sometimes LBRs cannot deliver the reminder effectively [Beigl, 2000, Dey and Abowd, 2000, Ludford et al., 2006, Marmasse and Schmandt, 2000, Sohn et al., 2005, Zhou et al., 2012]. Location plays a key role in supporting effective locationbased services. Sohn et al. [Sohn et al., 2005] proved that LBRs were regarded as useful in some cases. However, the detection of location for message delivery was often inaccurate, due to the limitation of technology and the complex changing environment. At the delivery moment of a LBR, the mobile environment can be complex, less controlled, and constantly changing. For example, construction might be blocking roads or access to doors, or the Euclidean distance to the desired location does not guarantee easy access, requiring that the geographic layout of the location [Ludford et al., 2006] be considered to generate an effective reminder. Researchers have addressed this challenge by taking advantage of the rich context available in the environment to define a more complex trigger situation [Dey and Abowd, 2000, Marmasse and Schmandt, 2000, Sohn et al., 2005, Zhou et al., 2012]. Furthermore, sometimes it can be difficult to detect the location information accurately. For example, LBRs are more accurate at detecting when users are approaching a location than when they are leaving a location [Marmasse and Schmandt, 2000, Li et al., 2005]. Additionally, only one location can be detected when two desired locations are near the each other [Marmasse and Schmandt, 2000], a phenomenon called location shadowing. The simplistic design of LBR delivery does not account for the complexities of location context.

With overwhelming information and technology, people today receive too many notifications [Church and de Oliveira, 2013, Sahami Shirazi et al., 2014]. People must spend extra effort 
responding to the notifications, which makes them turn off the services. Reminders aim to keep users engaged and to catch their attention, but with too many reminder services, people are getting tired of all the technology and information demanding their attention. Receiving reminders has switched from being something useful to something being overwhelming and disruptive. Consequently, people do not deal with reminders as seriously as they did before. As a result, technology is not as reliable as expected. In some cases, the reminder cannot be delivered effectively, due to the limitations of technology. While in other cases, people are not able to deal with the reminders effectively due to the overwhelming amount of information available today.

\subsubsection{Cognitive Offloading}

Today, people rely heavily on electronic reminders, which might attenuate people's ability to remember. Often, people rely more on using external memory aids, and less on their internal memories. Intons-Peterson and Newsome III [Intons-Peterson and Newsome III, 1992] determined that the use of external memory aids influences human internal memory. Herrmann and Petro [Herrmann and Petro, 1990] brought up the question of how the use or disuse of external memory aids might affect a person's memory skills. It has been argued that using memory aids might reduce internal memory skills since users do not need to employ these skills any longer. Payne [Payne, 1993] suggested a similar idea, the example being that the use of calendar aids to assist a person's remembering might actually contribute to them having trouble recalling the appointments in the calendar's absence. Ockerman and Pritchett [Ockerman and Pritchett, 2000] refer to this phenomenon as the "human over-reliance problem." In their study, they found that in a working environment, people over-rely on the task guidance system to follow the procedure and lose the insight of the original intent. 
More recently, this phenomenon has been defined as "Google effect" [Sparrow et al., 2011].

Today, people have the tendency to forget information that can be readily found on the Internet. Researchers found that the Internet has become a primary form of external human memory, where information is stored collectively, but outside of real human memory. People learn how to quickly access to the knowledge rather than learn the knowledge itself. As more information becomes available via external tools, people become progressively more reliant on it in their daily lives. Storm et al. [Storm et al., 2016] considered it "cognitive offloading," or that human memory is changing. Increasing reliance on the Internet is affecting humans' thought processes for problem-solving, recall, and learning. Researchers found that as memory is being extended outside, people today don't focus much effort on trying to recall something on their own, when technology is readily accessible.

At present, there is not too much research on LBRs, which might have contributed to addressing human memory in the design. My work explores this problem by redesigning the interface of LBRs, with the goal of avoiding the unintended results of attenuating man's ability to remember.

\subsection{Research Problem}

The current design for LBRs is largely based on "geofencing." During the setup of a reminder, users enter an address or a landmark and a radial distance (i.e., a "fence") around the location. A reminder is triggered and then delivered later, when the user either enters or leaves the border (i.e., "geofence") of the location. The setup stage of current designs offers a map view of the desired location (as shown in Figure 1.1).

The current interface design of LBRs fails to consider human prospective memory theory 


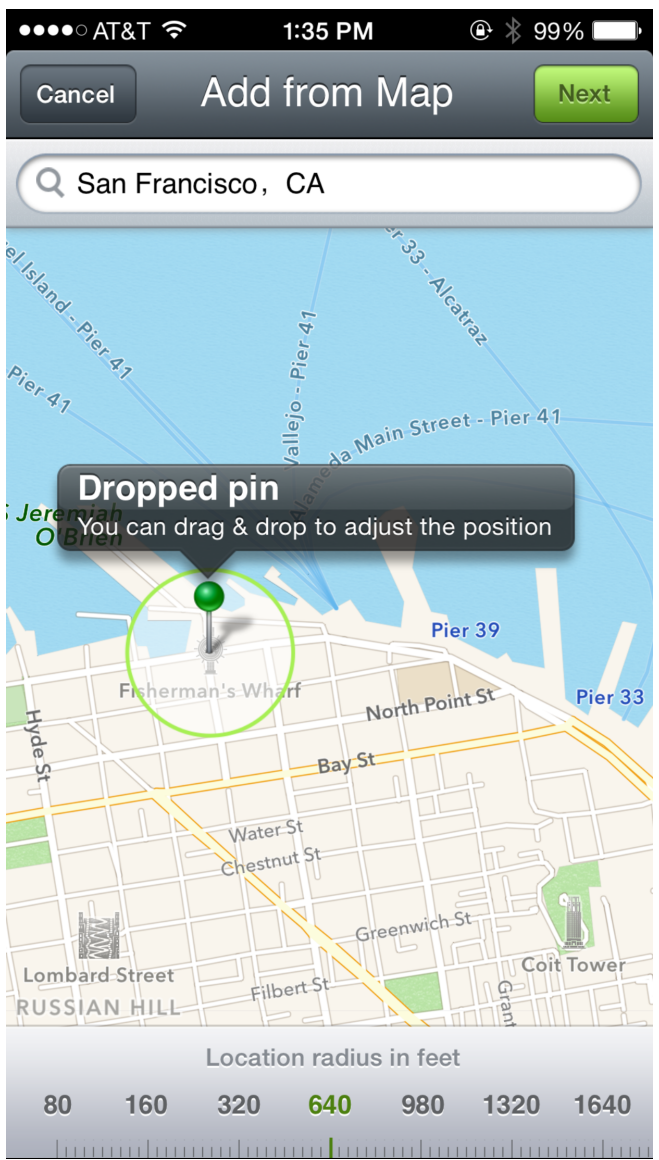

Figure 1.1: User interface in a typical reminder system showing how to set up up a locationbased reminder 
to make it work consistently with how people remember to-do tasks. Based on the theory of prospective memory, a person's prospective remembering would be highly facilitated if we brought the environmental context available at the retrieval stage to the encoding stage. The current encoding stage of LBRs provides a map-view of the desired location without benefiting prospective memory. A first-person view at the encoding stage is more consistent with how prospective memory works by offering a better match between the encoding and the retrieval stages.

Furthermore, location in LBRs, not only serves as a target place to execute the intended to-do task, but also serves as an external cue to trigger the intended to-do task. Prospective memory studies suggested that familiarity with the external cue influences prospective task triggering and remembering [Einstein and McDaniel, 1990]. Location information processing requires users to apply different types of spatial knowledge. A location can be easily recognized if people are familiar with the business logo of that location. The familiarity of a target location makes users perceive the environment differently, but these devices hardly consider our prior familiarity with the environment around us. For a person in his or her hometown, a relative description (e.g., "next to the high school") is enough to identify the destination and minimizes the demands of encoding the cue. But for a business traveler visiting a city for the first time, the same relative instruction is useless.

From a prospective memory point of view, there is a clear link between the setup stage and the delivery of the reminder. Beigl [Beigl, 2000] highlighted the importance of the setup stage in LBRs. According to Beigl, the way the notification is entered and the way the location is described and associated with the user's perceived location are two critical factors in the usability of LBRs. With the goal of making the technology work in a way that is more compatible with how human memory works, my dissertation focuses on the design of LBRs' encoding interfaces. I explore the impact of different encoding interface designs and the 
familiarity with location on recognizing desired locations and remembering associated to-do tasks. My work designs LBRs by considering human memory, so both technology and human beings can complement one another better.

\subsection{Research Questions and Hypotheses}

\subsubsection{RQ 1 - Matching Encoding and Retrieval}

RQ1: Does the method of matching the encoding stage and the retrieval stage facilitate human remembering location-based to-do tasks? Specifically, do people have a better recall performance on location recognition when the encoded location information is matched with the retrieval location information?

What if the setup stage showed instead a street view of the desired location? What if the setup stage took advantage of the compatibility often required between the encoding step and the retrieval step of prospective memory? Can I improve the reminders in LBRs, not by making the location-sensing technology better but instead by making the technology work in a way that is more compatible with how our memories work?

Research from prospective memory has shown that prospective remembering would be facilitated by increasing the match between the encoding and the retrieval stage [Einstein et al., 1996]. Einstein et al. [Einstein et al., 1996] suggest that one feature would be the environmental context in which the intended action is encoded and in which the action is performed. The target event may spontaneously prompt at the retrieval stage when the environmental context is reinstated. This theoretical finding might be especially useful in representing location information in LBRs at the encoding stage. 
Based on the theory of prospective memory, encoding and retrieval processes, I hypothesize that a first-person view of the desired location can improve location recognition and task recall for tasks to be performed at another location. In the first user study, I presented the design of a between subjects experiment that explores the role of the encoding stage in the remembering of eight to-do tasks and their locations three days later. The results showed that a street view of a desired location at the encoding stage significantly improves the location recognition and task recall at the retrieval stage.

\subsubsection{RQ 2 - The Impact of Familiarity}

RQ2: What is the impact of familiarity with cities and location businesses on performance at the retrieval stage?

Familiarity with a given location is known to affect spatial navigation [Thorndyke and Goldin, 1983] and prospective memory [Vortac et al., 1995], but it has not been explored with respect to LBRs.

On one hand, researchers found that familiarity influences the way people perceive spatial environments [Acredolo, 1982]. For example, perceptual icons such as business logos, or landmark buildings are usually the first piece of information learned by people. A place can be easily recognized if people are familiar with the business logo of that place. Otherwise, it takes some time to accumulate enough spatial knowledge of the environment to recognize a place. On the other hand, prospective memory studies suggested that familiarity with the external cue influences prospective task triggering and remembering [Einstein and McDaniel, 1990]. Therefore, I believe that the familiarity of a location influences the setup of LBRs at the encoding stage and the location recognition at the retrieval 
stage.

Based on the theory of human spatial knowledge and prospective memory, I hypothesize that to-do task recall and location recognition would be affected by the familiarity of the city being navigated and the location's business logo being recognized. I conducted a second empirical user study exploring the impact of familiarity on performance in location-based to-do tasks by measuring recognition of a location and recall of an associated prospective task. I found that displaying a business logo on a map can be an effective cue to enhance location recognition in a familiar city, while street-view images of the location provide an effective cue for location recognition in an unfamiliar city.

\subsection{Contribution}

I have made the following contributions in this dissertation:

- I propose a theoretical framework by matching human prospective memory with LBRs. This framework opens a new research area by applying human memory in the design of LBRs to alleviate the "human over-reliance" problem. Research questions are identified in this framework.

- I demonstrate that providing a first-person view (street view) at the encoding stage for LBRs has a significant effect in supporting how people recognize that location and recall the task at a later retrieval time. This work provides evidence on how to use prospective memory to improve the design of LBRs.

- I demonstrate that familiarity with the location and city has a significant impact on performance of location recognition. I found that street view images have a significant 
advantage in supporting location recognition in an unfamiliar city, and shows a benefit for people recognizing unfamiliar businesses.

- I provide design implications for future LBRs. I conducted two comparative betweensubjects experiments to understand how people remember and recall location-based to-do tasks without the aid of reminder services. I conducted statistical analyses to deliver future design implications for LBRs.

- I designed a new LBR prototype by applying theoretical research findings of human prospective memory. This new interface supports reminding people to recognize a location in a more natural way. This design uses technology to benefit human memory, so technology and humans can work together more effectively, to avoid the "overreliance on technology" effect.

\subsection{Overview of the Dissertation}

Chapter 2 presents the theoretical foundation of my work. It is a review of the literature of human prospective memory theory and human spatial knowledge. Chapter 3 reviews the prior studies on existing reminders. This literature review comes from human memory aids, general reminder applications, and the design of existing LBRs. Chapter 4 reports a usability evaluation study of an existing LBR. The results support that current design of LBRs fail to consider human memory. Chapter 5 describes an online survey and proposes a classification of different uses of locations for location-based to-do tasks.

In Chapter 6, I present the theoretical framework for my dissertation by matching prospective memory with LBRs. This framework identifies two main research questions that this dissertation has answered. In Chapter 7 and Chapter 8, I describe the methodology I use in 
two empirical experiments, giving details of the experiment design and procedure, reporting data analyses, results and design implications. Chapter 9 covers the design, implementation, and usability evaluation of a LBR prototype. Chapter 10 highlights the conclusion of my work and describes possible future work in this domain. 


\section{Chapter 2}

\section{Theoretical Foundation}

There is a vast amount of research studying human memory. Human information processing involves attention resources to perceive information, and then process the information into working memory or further encode it into long-term memory [Wickens, 1992]. Research typically differentiates short-term memory from long-term memory, and describes working memory as an active-inactive memory phenomenon. Another memory classification refers to the difference between retrospective and prospective memory. Retrospective memory is concerned with past events such as remembering an adventure in one's childhood, while prospective memory is to remember an intended action in the future.

My dissertation specifically focuses on the prospective memory and its use in LBRs. My work explores the role of prospective memory in supporting the design of LBRs. From a prospective memory perspective, LBRs are a type of external memory aid, which is used to support people remembering to-do tasks associated with location information. My work contributes on how to improve the design of LBRs, using research from the areas of prospective memory. While there is work in context-based processing that is closely relevant to my work, my interests are not in improving the context sensitivity of LBRs. Instead, I investigate how 
to improve the effectiveness of LBRs by increasing the reliability of prospective memory to make LBRs work more consistently with how human memory works.

\subsection{Prospective Memory}

Prospective memory is a form of memory that helps people to remember actions, intentions, or things to do at a future time. As proposed by Ellis [Ellis, 1996], a successful prospective task follows a four-stage procedure. The stages are: a) intention formation, which requires human attention resources to process and encode the task; b) intention retention, which accounts for the interval from remembering the task until the moment of retrieving it; c) intention retrieval, which involves human prospective memory to recall the task at the right moment; and d) intention execution, which means the execution of the intended action.

\subsubsection{Prospective Memory Tasks}

There is a wide variety of prospective memory tasks in people's daily activities. The most common distinction is between time-based and event-based tasks [Einstein and McDaniel, 1990]. Time-based tasks are those to be performed at a specific future time; for example, a medical appointment at $10 \mathrm{am}$. Event-based tasks are to be performed when a particular situation or event occurs; for example, driving past the local library cues you to remember that you need to return an overdue book. The inherent difference between these two types of tasks is in the cognitive retrieval process [Einstein et al., 1996]. Event-based tasks rely on an external cue to trigger the intended action and are possibly retrieved through a spontaneous process when people notice the external cue associated with the intended action. Time-based tasks do not have these external cues, relying instead solely on the self-initiated retrieval process 
by paying attention to time. Location-based to-do tasks are examples of event-based tasks.

\subsubsection{External Cues}

Vortac et al. [Vortac et al., 1995] divided prospective memory tasks into two major components. One component is the external cue that triggers the intended action, the other one is the content of the action. For example, if the task is to buy fruits at a grocery store, the grocery store is the external cue, noticing the grocery store on the street might be the trigger, and the intended action is to buy the fruits. Both components, cue and action, are required to successfully complete a prospective task.

The memory cue is one of the keys to successfully supporting prospective remembering [van den Hoven and Eggen, 2014, Vortac et al., 1995]. Researchers have concluded that some attributes of external cues, such as the distinctiveness and the associativity with the intended task, are inherently important to support task retrieval [McDaniel and Einstein, 2000]. If external cues are salient, distinctive, and highly relevant with the intended task, they will more effectively trigger the intended action.

Research in prospective memory has created a deep understanding of the effectiveness of external cues and the remembering of intended actions, but not much of that research has been applied in the research of LBRs. In the context of LBRs, Sohn et al. [Sohn et al., 2005] found that location itself serves more like a reminder cue more than just the place of the task execution. 


\subsubsection{Encoding and Retrieval}

Encoding and retrieval are the two major stages in successfully completing a prospective memory task. Einstein and McDaniel [Einstein et al., 1996] suggested that prospective remembering would be especially sensitive to the match of features present at the encoding and retrieval stage. People should have a better prospective task recall performance if there are more similarities between the encoding stage and the retrieval stage. That means that if the environmental context, i.e., the street view of the location, can be presented at the encoding stage, then it would facilitate prospective remembering. To make the LBRs' use intuitive, designers should make the interface of LBRs to match the way humans remember.

\section{$2.2 \quad$ Prospective Remembering}

The inherent difference between time-based prospective memory tasks and event-based prospective memory tasks is in the retrieval process. Event-based prospective memory tasks have external cues that can support and guide remembering, while time-based prospective memory tasks don't have these cues. As a result, time-based prospective memory tasks are dependent on a self-initiated monitoring retrieval process while event-based prospective memory tasks could possibly depend on an environmental cue automatically triggering the to-do task. A location-based to-do task is a typical type of event-based prospective memory task with the location serving as an external cue. Noticing the location signals people that it is time to perform the intended action at this location. My work focuses only on the cognitive retrieval process for event-based prospective memory tasks. 


\subsubsection{Cognitive Retrieval Process}

In theory, there are two ways of cognitive processes that can successfully trigger the eventbased prospective memory tasks. The retrieval of the event-based tasks generally supports a multi-process framework of prospective memory in two ways [McDaniel and Einstein, 2000, McDaniel et al., 2004, Einstein and McDaniel, 2005]. One is termed "reflexive-associated process," which indicates that no attention resources are required, and it can only be effective when the cue is distinctive. This is usually what people refer to colloquially as "it just popped into my mind." When forming an intention for an event-based prospective memory task, people create an associative link between the external cue and the to-do task. Noticing the cue automatically activates the associative link to the intended action and brings it to the conscious awareness level. The other is called a "cue-focused process" (also known as noticing + search process), and it is assumed that people need to commit attention resources to monitor external cues. Upon encountering the external cue, people would have to interrupt the ongoing activity. This noticing of the external cues may not trigger the task directly, but may elicit feelings of familiarity and then stimulate a further directed search in the memory about the cue. The main difference between the two processes is whether they involve human attention resources or not.

\subsubsection{Implementation Intention}

Research evidence clearly shows that prospective memory recall performance benefits from implementation intention (i.e., "When I see X, I will do Y"). Implementation intention decides when, where, and how to implement one's goal in advance [Gollwitzer, 1999]. Linking the expected critical situation to goal-directed response (i.e., "Whenever situation X arises, I will make the goal-directed response Y"), can help people more effectively complete a 
task. Gollwitzer [Gollwitzer, 1999] noted that because implementation intention implies a future situation in the environment, when the situation becomes accessible, the mental representation of the situation is highly activated. Dismukes [Dismukes, 2010] suggested that implementation intention provides a powerful way to improve prospective memory performance. When applying implementation intention strategy in remembering prospective memory tasks, people encode a specific time and place, and identify the environmental cues likely to be present at the retrieval stage. This method is especially helpful with people remembering location-based to-do tasks.

\subsubsection{Familiarity of External Cues in Prospective Remembering}

Successful event-based prospective remembering is heavily dependent on the ease of identifying the external cues at the retrieval stage [Vortac et al., 1995]. Researchers revealed that different prospective memory tasks are associated with different conscious experiences [Graf and Uttl, 2001]. For example, remembering to highlight the keywords while reading through a paper; this prospective memory task (to highlight keywords) is a short-term task remaining active in the conscious awareness all the time. Real-world prospective tasks usually occur while people are engaged in other activities. Most prospective tasks in everyday activities are usually planned and scheduled in advance. Finishing all four stages takes some time, varying from a couple of days to a few months. In fact, the prospective memory tasks are usually out of conscious awareness most during the time from forming the intention until executing the intended to-do task. Instead, this period is filled with other activities until the appearance of the external cue. Triggering the intended to-do task relies on attentional capture by the external cue. Vortac et al. [Vortac et al., 1995] demonstrated that the success of intended to-do task retrieval may depend on the attention-grabbing ability of the external cue when it appears. The key to a successful task retrieval is whether the cue could succeed 
in bringing the previously intended action back into conscious awareness.

Several studies investigated the effectiveness of external cues to explore how the cues might influence a successful retrieval [Dismukes, 2010, McDaniel and Einstein, 2000]. Distinctive, unusual and salient cues are generally effective to retrieve the to-do task. McDaniel and Einstein [McDaniel and Einstein, 2000] indicated that distinctive external cues may engage an involuntary orienting process in supporting prospective remembering. If the external cues are salient or unusual, it would be easier for people to switch attention from the ongoing task to the to-do task. A second key factor influencing the effectiveness of external cues is the association between the external cue and the intended action. The "reflexive-associated process" is more likely to be the remembering route when the cue and the intended action are highly paired.

Research has shown that the familiarity of the external cue has a significant influence on prospective remembering. Researchers conducted experiments varying the familiarity of the external cues, and concluded that uncommon and unfamiliar external cues produce better prospective remembering performance [McDaniel and Einstein, 1993]. In their study, external cues successfully triggering the intended to-do tasks was considered as a successful prospective remembering. Further exploration indicated that common and familiar external cues have many preexisting associations, and these may interfere with the intended to-do task. By contrast, uncommon and unfamiliar external cues have few pre-existing associations with little interference. For example, seeing a shopping center may not successfully trigger the to-do task since there are so many tasks that can be done at a shopping center, however seeing a dry cleaner (laundry) may successfully remind the person to pick up the laundry. 


\subsection{Prospective Forgetting}

Cockburn [Cockburn, 1996] concluded that prospective memory failures can occur under two situations: the breakdown of the encoding stage (poor encoding) or the failure access of the retrieval stage (cues fail). An inadequate encoding of intention can lead the record of task and external cue to be vague and inaccurate. It is likely that people fail to recognize or respond to the external cue in the context, which results in a failure of the retrieval. If the intention is there, but the intended action is not well encoded, it is likely that the external cue can trigger the memory that something should be done-but what should be done is not recalled.

\subsubsection{Poor Encoding}

Poor encoding is one of the common reasons for lapses in human memory. Elsweiler et al. [Elsweiler et al., 2006] indicated that participants often said, "I didn't really pay attention to it" or "I didn't make an effort to remember" to respond to the memory failures. They demonstrated that one reason people have trouble with remembering is the poor encoding process. Vemuri and Bender [Vemuri and Bender, 2004] concluded that people encode information in a summary form without filling out the details. People tend not to dedicate much effort to the initial encoding process, which sometimes results in memory failure.

\subsubsection{Cues Fail}

Not recognizing the external cue at the retrieval stage is another reason for a prospective memory failure. The pair between the external cue and the intended action subsides to levels below conscious awareness. To successfully retrieve a prospective memory task, the cue is 
used to activate the associative link between the cue and the intended action, and to bring the intended action into the conscious awareness level. However, cues are sometimes not noticed for unexpected reasons [Dismukes, 2010]. Moreover, even if the external cues could be noticed, sometimes they couldn't be processed into human attention when it occurs due to the complex environmental context [Einstein et al., 1996]. When people are highly involved with an ongoing activity, or simply tired, no extra attention resources can be allocated to react to the external cue.

\subsection{External Memory Aids}

Because of typical memory failures, knowledge and mobile workers rely on external memory aids to remember things to do in the future. These aids typically take the form of calendar alerts, to-do lists and electronic Post-it notes in our mobile devices. They are often paired with mobile alerts intended to remind us to do things when we approach a certain location.

Memory aids have been studied for a long time [Harris, 1978]. Internal memory aids involve reliance on one's own internal memory, such as mental rehearsing, mental images, or unique associations. External memory aids rely on the use of external mechanisms; examples include creating a shopping list, setting a timer, or putting something in a special place (e.g., lunch bag in the garage door). Several studies have investigated the memory aids people use, and have concluded that external memory aids were used more often than internal memory aids due to their accuracy and reliability [Harris, 1980, Intons-Peterson and Fournier, 1986].

LBRs are considered to be a type of external memory aid helping people remember and retrieve future to-do tasks [Harris, 1978]. Some researchers have concluded that external memory aids function as cues in supporting task recall for encoding, recoding, reorganizing, rehearsing, and retrieval [Intons-Peterson and Fournier, 1986]. Intons-Peterson and New- 
some III [Intons-Peterson and Newsome III, 1992] have demonstrated that human memory affects the use of external memory aids; we seek to understand the role of prospective memory in LBRs.

Usually, external memory aids serve as a retrieval role. Intone-Peterson and Fournier [Intons-Peterson and Fournier, 1986] demonstrated that sometimes external memory aids also play a mental rehearsal role. They found that taking notes of a list of grocery store items at the encoding stage facilitates remembering of the information, even if the notes were not available at the retrieval stage. In my work, I consider whether displaying the retrieval context at the moment of capturing the prospective task would improve the performance at the retrieval stage.

Morita [Morita, 2006] suggested that reminders which referred only to external cues might also benefit memory recall. Theoretically, successful prospective memory requires the external cue to be noticed. Morita [Morita, 2006] predicted that the reminders can be effective if they can activate the representation of the external cue. Guynn et al. [Guynn et al., 1998] studied reminder effectiveness by simulating real world prospective memory context where people are busily engaged in activities and must be interrupted to switch to the intended task. The results indicated that in order to make the reminder effective, it must activate the association between the external cues and the intended to-do tasks.

\subsection{Spatial Knowledge}

Spatial knowledge is knowledge accumulated over time that allows people to navigate their physical environment. Researchers have categorized spatial knowledge into three types: survey knowledge, procedural knowledge and landmark knowledge [Thorndyke and Goldin, 1983]. People acquire these different types of spatial knowledge from diverse types of artifacts and 
experiences, such as maps, navigation experiences, descriptions, photographs and so on.

\subsubsection{Types of Spatial Knowledge}

Survey knowledge refers to knowledge of the topographic properties of an environment [Thorndyke and Goldin, 1983]. Map data is an example of survey knowledge presenting to users object locations and global spatial relations. Procedural knowledge includes first-person information about traveled routes, typically presenting information from the perspective of an individual navigating within the environment [Thorndyke and Goldin, 1983]. Procedural knowledge encodes the spatial relationship between two points with the route connecting them. Landmark knowledge presents information about the visual details of specific locations in the environment [Thorndyke and Goldin, 1983]. People usually acquire landmark knowledge from perceptual icons [Thorndyke et al., 1980], images, buildings, or signs. In my work, I considered business logos to be perceptual icons that serve as proxy for a level of familiarity with a business location.

One or more of the three types of spatial knowledge described above may be available for a given location within an environment [Thorndyke et al., 1980]. Studies have investigated the differences between survey and procedural knowledge [Thorndyke and Hayes-Roth, 1982]. Survey knowledge leads to a two-dimensional bird's eye view of the environment. The advantage of survey knowledge, typically acquired from map learning, is that it captures global relationships, such as estimating Euclidean distance between locations and perceiving relative positions of locations. Acquiring procedural knowledge leads to a three-dimensional egocentric view of the space, allowing people in the field to relate the surroundings to their position. 


\subsubsection{Familiarity of locations in Spatial Knowledge}

The different types of spatial knowledge are used differently in familiar and unfamiliar environments. Perceptual icons (landmark knowledge) are usually the first pieces of information learned by people when encountering a new environment [Thorndyke et al., 1980]. As people become familiar with the environment, perceptual icons are supplemented with names and locations. Then procedural knowledge is accumulated by associating these recognizable perceptual icons with route information. Survey knowledge is constructed by learning the relations between these perceptual icons in more abstract terms (i.e., cardinal directions).

As people become familiar with an environment, the type of spatial knowledge used might be a combination of the three. A mental map can be formed by gradually acquiring elements from the environment: first landmarks, then routes, then connecting them together with survey information [Tversky, 1993]. Once people learn the environment, they would have an accurate mental map which contains vivid visual imagery. Thorndyke and Hayes-Roth [Thorndyke et al., 1980] also found that survey knowledge improves with the increase of time of residence in an environment. In an unfamiliar large-scale environment, survey knowledge is not enough; navigation information is required to get around.

Hirtle and Sorrows demonstrated that images can be as useful as maps to locate particular buildings in the environment [Hirtle and Sorrows, 1998]. Images of buildings make it easy for people to learn location information, especially when the buildings are distinctive. Hirtle and Raubal also pointed out that the images shown by Google Street View allow a person to identify the target location in the environment [Hirtle and Raubal, 2013].

Numerous studies have confirmed the positive relationship between familiarity and accuracy of spatial cognition. O'Neill found that familiarity with the environment generally enhanced navigational performance [O'Neill, 1992]. Their experiment investigated the effects of degree 
of familiarity on spatial cognition and navigation. Navigational performance data improved significantly as participants became increasingly familiar with the setting. Also, the effect of familiarity with a building was found to facilitate spatial orientation when walking through a large building complex; this effect persisted at a re-test one month later [Gärling et al., 1983]. Researchers found that pointing to highly familiar places produces less error than pointing to less familiar places [Golledge, 1992]. Therefore, familiarity appears to influence the accuracy of recognizing a place and spatial judgments with respect to that place.

\subsubsection{Naive Geography}

Naive geography represents a common-sense view of the geographic world. Users without training in geography are considered to be naive users. Naive geography mainly addresses naive users' spatial cognition for how space is accessed and processed. They tend to acquire common-sense knowledge, which is usually expressed in linguistic terms such as a place name (e.g., Grand Canyon, White House) and spatial relations to link these places together such as "in front of" or "near" [Jiang and Yao, 2006]. The naive geography framework pointed out that when people use multiple conceptions of space, they can think of locations as both points and areas [Frank et al., 2004]. Naive geography also includes some other observations [Hirtle, 2011]. For example, naive users often cannot accurately tell how far 1,000 feet is, but they can tell how many blocks it is to a location or how many minutes it takes walking to reach a destination.

\subsubsection{Hierarchical Structure of Location}

Spatial knowledge in nature is perceived hierarchically [Darken and Sibert, 1996]. Stevens and Coupe [Stevens and Coupe, 1978] found that spatial information is also stored hierar- 
chically. Each larger, general place is organized around a few smaller more specific places. Directions and distance relations connect each place together to form the hierarchical structure. For example, people do not remember the absolute locations of certain cities, but they remember the states where the cities are located.

\subsection{Theoretical Foundation for User Study 1}

In summary, performance in a prospective to-do task depends on the person recognizing the location and recalling the task to be done at that location. Recognizing the location can be aided by a reminder and by a close match between input at the time of encoding and the cue at time of retrieval. My work leaves the actual reminder for future studies. Currently, I focus on how people can effectively recognize the location and then recall the associated task without the aid of a reminder.

Users view their surroundings from an egocentric perspective. However, the current design of LBRs gives an allocentric (bird's-eye) view of the target location during the encoding stage. To set up a location in the current design of LBRs, people must translate the target location between the two views in their minds. Unfortunately, shifting from a perspective "above" the environment to a perspective "within" the environment is difficult [Frank et al., 2004, Thorndyke and Goldin, 1983]. Researchers found that spatial task performance declines when it requires such a change of perspective [Thorndyke et al., 1980]. This change of perspective is more difficult (or maybe impossible) when the person is in an unfamiliar space.

Prior work on prospective memory and spatial knowledge informs the hypotheses for my first user study. A good match between encoding and retrieval stages improves location recognition, which often leads to a better recall of the associated task. According to prospective 
memory theory [Einstein et al., 1996], people's prospective remembering would be highly facilitated if we brought the environmental context available at the retrieval stage to the encoding stage. LBRs have adopted a birds-eye view of the desired location, but first-person view of those would be more appropriate. The technology for doing so is readily available.

\subsection{Theoretical Foundation for User Study 2}

My second user study is interested in exploring how familiarity impacts the recognition of the location and the recall of the task. My work studied familiarity of the hierarchical structure of space by considering two levels: city and place. Darken and Sibert [Darken and Sibert, 1996] defined a place as a distinct, recognizable location or region of a larger space associated with a landmark. My second study investigated familiar and unfamiliar places in familiar and unfamiliar cities.

Familiarity increases landmark, procedural, and survey knowledge with the environment, which leads to an increase of knowledge of the place. With this increased knowledge, location recognition is improved because participants can build proximity information (e.g., "it is next to the bakery") into their encoding of the desired location. Familiarity with a business logo (perceptual icon) is part of landmark spatial knowledge which is the first stage towards developing a strong spatial knowledge. So even in an unfamiliar city, recognition of familiar business logos can help improve performance in location recognition. Finally, survey knowledge improves with the increase of time of residence in a city, so performance in the place of residence should be different in unknown cities.

Learning a location from a bird's-eye-view (Google Maps) requires people to use their survey knowledge, while processing the images captured in an allocentric way (e.g., Google Street View) requires people to use their landmark knowledge. Interactive street view (Google 
Street View supporting interactions) combines the use of procedural knowledge and landmark knowledge. I use these three encoding methods (Map, Street and Interactive Street views) in the second study to understand how spatial cues during prospective memory encoding stage are used in an LBR system in familiar and unfamiliar spaces. 


\section{Chapter 3}

\section{Prior Work in Reminders}

Due to the nature of human forgetting, external memory aids have been designed to better support human remembering. In this chapter, I review prior work of different types of reminders that support human memory.

\subsection{Human Memory Aids}

Most external memory aids aim to support reminiscing and retrospective memory, with little research focused on prospective memory. Forget-me-not [Lamming and Flynn, 1994] was a conceptual prototype that exploited some knowledge of human episodic memory. It was designed to help people deal with daily memory problems. Based on the core idea that physical context can be a powerful cue for recall, this device aimed to capture important data and context from user's personal life, and later organized these data into a form that mimicked the episodic memory structures created by the user. In this way, the forget-me-not prototype could support human memory and retrieve appropriate information when a user only remembered the context of the event. Although a great deal of technology and infrastruc- 
ture was not yet available at that time, the idea pointed out the potential future direction of memory aids. Memojog [Morrison et al., 2004] was an interactive memory aid for elders with normal cognition, aimed to help elders remedy everyday memory problems. It was able to automatically monitor users' responses and respond to the user accordingly. DeVaul and Corey [DeVaul et al., 2003] designed a wearable system - memory glasses - and concluded that providing subtle visual cues can be as effective as a just-in-time memory retrieval aid, especially when overt cues are distracting and inappropriate. They suggested that subliminal cues without conscious attention or awareness would also be processed by the human brain, and affect the task performance. Vemuri and Bender [Vemuri and Bender, 2004] brought up a new generation of personal memory aids. Their method applied ubiquitous recording technology and extracted valuable content and context to support memory recall. The prototype they designed, iRemember, is a search-able personal archive of everyday experiences. Their study concluded that providing more details at the time of encoding could leave a stronger memory trace for future retrieval. Vemuri and Bender considered this preparing process as a memory-encoding aid. They concluded that the more details one encodes, the more association paths one can have in memory.

\subsection{Reminder Applications}

With the everyday use of reminders, several studies applied prospective memory model into the design of existing reminders. Payne [Payne, 1993] provided a cognitive analysis between the design of calendars and the task of prospective remembering. He analyzed the task structure based on the prospective memory four-stage procedure (discussed in section 2.1), and further discussed how the use of calendars affects the structure of remembering the task. First, using calendars influences the intention formation. The intention must be 
named and described to encode the intention into the calendar, which might influence the mental encoding. Then, the use of calendars also affects the intention retention stage between encoding and recall. He found that when one started to use the calendar, they consequently (he assumed) had a great deal of trouble remembering appointments. At the recall stage, electronic calendars provide alerts to trigger the task, and to guide users to carry out the to-do task, but it can typically only be set for precisely scheduled events, which constrains the structure of prospective memory tasks.

When sending email became an everyday activity to knowledge workers, several researchers investigated the prospective information in email. Gwizdka [Gwizdka, 2001] found that email is a common tool people use to handle prospective information. Handling this type of event involves human prospective memory. By linking the prospective memory five-stage model (which added the evaluation of the execution as a final stage) to the functions supported by the email system, Gwizdka identified that the management of future messages is inadequately supported by email. In a more general way, some researchers tried to understand the role that memory plays in the use of email system. Elsweiler et al. [Elsweiler et al., 2006] designed Memomail, which facilitates users' memories to support email retrieval. Since people tend to remember the context, this Memomail could help people recover from retrospective memory lapses by incorporating fragment of contextual information. The contextual information may be serving as external cues that trigger memories, which is similar to the practice of people leaving objects in obvious places as cues to remind them to perform tasks.

Some researchers extended the calendar design to external memory aids for brain-injured people. Thone-Otto and Walther [Thöne-Otto and Walther, 2003] indicated that external memory aids have hardly been used in the therapy of memory-impaired patients. In their work, they defined the problems of brain-injured patients and the demands on external memory aids on the basis of the prospective memory five-stage model, and developed a 
system taking into account these aspects. They concluded that the prospective memory model can serve as a helpful background to understand electronic memory aids. McDonald et al. [McDonald et al., 2011] analyzed the prospective memory deficits for brain injured people, and investigated the effectiveness of Google Calendar to support different prospective memory components. They concluded that Google Calendar has considerable potential to be used as a novel external memory aid in the rehabilitation of brain-injured people.

\subsection{Location-based Reminders (LBRs)}

Different kinds of reminders are widely used nowadays to support people getting things done, such as setting calendar alerts, sending oneself emails or messages, and making to-do lists. These existing reminders lack the ability to trigger the reminder at an appropriate place. Rao and Minakakis [Rao and Minakakis, 2003] suggested that location plays a key role in determining the type and nature of human activity. For example, a grocery list reminder is more helpful while the user is near a supermarket on their way home, rather than while at work or after arriving home. In many cases, it is believed that location is more appropriate than time to trigger the intended action. This section reviews several designs of LBRs and discusses the existing research challenges in studying LBRs.

\subsubsection{Early Design of LBRs}

Researchers developed several prototypes to prove the usefulness of LBRs. The Stick-e Note was considered an early context-aware reminder application to support prospective tasks [Brown, 1995]. Brown applied the Post-it metaphor in the digital environment, where users could place a message at their current location using GPS-enabled PDAs. Whenever the 
user returned to the same position, the message was triggered and displayed to the user. In a Stick-e document, the context is a combination of elements from the environment, such as location, time, adjacency of other objects, temperature states and so on. The Stick-e document concentrated on reminding people to do something in the future. Beigl [Beigl, 2000] found that in many cases it is often space rather than time that serves to remind people of a task. He developed a prototype, Memoclip, to actively remind a user of tasks depending on their locations. This prototype allowed users to create a subjective description for each place and to use their cognitive maps to perceive the location. In his work, he found two factors influencing the usability of a location-based reminder: one is the way notification information is entered, and the other is the way location is described and associated with a user's perception of a location. ComMotion [Marmasse and Schmandt, 2000] was designed to provide just-in-time information delivery based on people's physical locations. This prototype could gradually learn about users' travel patterns and allow users to label a frequently visited location. Users could attach a to-do list with a relevant location, and an audio alert would be sent to users based on their location.

\subsubsection{LBRs to Remind Daily To-do Tasks}

My work focuses on how a location-based reminder can be triggered to remind people to complete a daily to-do task. Place-Its [Sohn et al., 2005] and PlaceMails [Ludford et al., 2006] were two significant LBR prototypes aiming to support people's daily to-do tasks. Place-Its was a LBR running on a mobile phone, addressing the rich contextual information. The reminder could be triggered upon arrival or departure of the associated place. An exploratory user study was conducted to understand how it could be used to support people's daily to-do tasks. They also found that location was not only considered as a physical place to execute the task, but could be widely used for identifying other context. For example, if a 
to-do task is to pass a colleague a book, the office will be the place people set this reminder, since it is in the office that people will likely be when seeing their colleagues. Ludford et al. [Ludford et al., 2006] developed an LBR called PlaceMail, and demonstrated its utility in supporting how people managed their everyday tasks. They demonstrated that setting an effective, accurate reminder was very complex, and discovered that to enhance the delivery accuracy of location-based messages, LBRs should consider the users' travel patterns and the geographic layout.

\subsubsection{LBRs to Support Social Life}

Some LBRs were designed for very particular purposes. GeoNotes [Espinoza et al., 2001] studied the social aspects of location-based information systems, and it encouraged users to create their own content to achieve a social and dynamic information space. Some contextaware reminders were designed to support on-campus social events. E-graffiti was a contextaware mobile social system [Burrell and Gay, 2002], with the ability to detect users' physical location on a college campus, and display text notes to the user based on their location, to further support students communicating with one another via note-posting on campus. ActiveCampus [Griswold et al., 2004] was a context-aware reminder system, which was restricted to a university campus and aimed at enhancing campus learning experiences. Users could use this prototype to set reminders to be triggered at a predefined location on campus, as well as to reveal nearby friends, and interesting events to support serendipitous experiences. There are other reminders designed to benefit location-based services. NAMA [Kwon et al., 2005] was a context-aware personalized reminder system based on the identification of a purchase need. By using the contextual information, the system could dynamically and proactively identify users' current purchase needs and send users reminders. Liu et al. [Liu et al., 2011] proposed a LBR to make the advertisement and product promotion more 
effective. Users could actively capture the images for a favorite product, and a reminder would then be delivered when the user was near the place where the product was being sold. They found that associating to-do tasks with physical locations provided a more convenient means for task management than conventional time-based ones. These LBRs provided a different perspective to understand the importance of location-based information in people's daily lives.

\subsubsection{Delivery mechanism of LBRs}

One of the unresolved research challenges in studying LBRs is that the current location-based delivery method sometimes is not accurate. Sohn et al. [Sohn et al., 2005] demonstrated that LBRs were regarded as useful, but the location detection for message delivery was inaccurate. It is not always easy to detect an exact location. For example, it is more accurate for LBRs to trigger a reminder while users are approaching a location than it is to detect users' departures [Marmasse and Schmandt, 2000]. Li et al. [Li et al., 2005] found that due to GPS latency and tracking of users' locations while inside of the "geofence," it was difficult to accurately detect when users were leaving a location. In addition, only one location could be detected when two desired locations were near the each other, a phenomenon called location shadowing [Marmasse and Schmandt, 2000].

The simplistic design of "geofence" does not account for the complexities of location context [Wang and Perez-Quinones, 2015]. Ludford et al. [Ludford et al., 2006] demonstrated that setting an effective reminder delivery point is very difficult. They suggested that the effective reminder delivery should be based on patterns of human motion, such as the geographic layout of the space, and the users' travel patterns. Inspired by their work, Tu et al. [Tu et al., 2013] developed an LBR prototype that considers users' routes instead of 
the "geofence" radius for the delivery of the reminder. They argued that the current fixed Euclidean distance method is insufficient to reflect the real location context of where users are situated. Their prototype, iReminder, which can predict users' future routes by collecting their daily trajectory data, could deliver reminders more appropriately. However, predicting users' future routes has always been argued as a big privacy and ethical issue [Wang and Loui, 2009].

To deliver an effective reminder, LBRs also need to be made more context-aware. Contextawareness aims to monitor the changing contexts of the user and adapt to users' activities appropriately [Abowd et al., 1999]. Several studies tried to make use of the rich context in the environment to determine an effective delivery situation. CybreMinder [Dey and Abowd, 2000] was an early-stage context-aware system for supporting reminders. By making use of rich context in the environment, the goal was to provide reminders appropriately. This prototype utilized complex situations to define a reminder delivery situation, including location, time, activity, identity, physical environmental conditions, and so on. This tool enhanced the user's ability to deal with reminders more effectively by presenting signals at appropriate times in appropriate locations. Place-Its [Sohn et al., 2005] addressed rich contextual information, with reminders delivered upon arrival or departure of the associated place. Lin et al. [Lin et al., 2012] designed a LBR with the purpose of complimenting the blind spots of GPS location sensing. They argued that the fact that location sensing merely relied on the GPS and limited the effectiveness of the system, so they designed a new LBR to be effective in both indoor and outdoor environments by using new technology. Zhou et al., [Zhou et al., 2012] created a context-aware reminder system for elders, and considered other factors to trigger the reminder, including the interrupt level of users' current activities and the urgent level of the intended task. While this approach enriches the context of the decision for the reminder, it also requires the users to put forth a lot of effort when setting up 
a reminder. Cakmak and Basoglu [Cakmak and Basoglu, 2012] indicated that users do not like a long process for setting up a simple reminder, so the acceptability of a more complex setup does not seem promising.

\subsubsection{User Evaluations of LBRs}

Several user studies were conducted to have a better understanding of what factors influence the use of LBRs in a mobile context. When moving in a natural environment, people usually have a mental plan on how to navigate from place to place with what actions and stops can be made on the way. Tamminen et al. [Tamminen et al., 2004] found that in their study, participants often popped in somewhere to do some tasks on their way to the primary destination. They also suggested that it is important to allow users to choose or create a route that is not only the shortest or the fastest, but otherwise rewarding. Kaasinen [Kaasinen, 2003] studied users' needs for location-aware mobile services and concluded that location-aware information was expected to be especially useful in special situations, for example in an unfamiliar environment. Li et al. [Li et al., 2005] investigated the issue for LBRs of how best to provide ubiquitous support for setting, detecting and delivering a location-based reminder to people. Users demand a timely and reliable LBR. They also have the requirement to cover a wider location to set and trigger the reminder. For example, people need the LBRs to detect when one is leaving a location. CakMak and Basoglu [Cakmak and Basoglu, 2012] explored factors that influence a user's intention to use LBRs. They found that with more time saving and less memory load involved, people would be more likely to perceive a location-based mobile application as useful. People would like to get more work done in less time, and spend less memory for less important issues. 


\subsubsection{State-of-the-art LBRs}

Today, the iOS system has Reminders which supports location-based reminder services. iOS Reminders allows users to specify a particular location on the map using a "geofence" design. Figure 3.1 shows an example of setting up a reminder to "get cash from Wells Fargo Bank." This design shows a map view of the particular location. Cortana is a popular LBR on Windows phones (See Figure 3.2). Google Now supports LBR features on the Android platform (see Figure 3.3). These two reminders give users the option to select any available place rather than requiring users to specify a particular place. However, this design provided limited information about the contextual environment for users to learn the desired location at the encoding stage. 


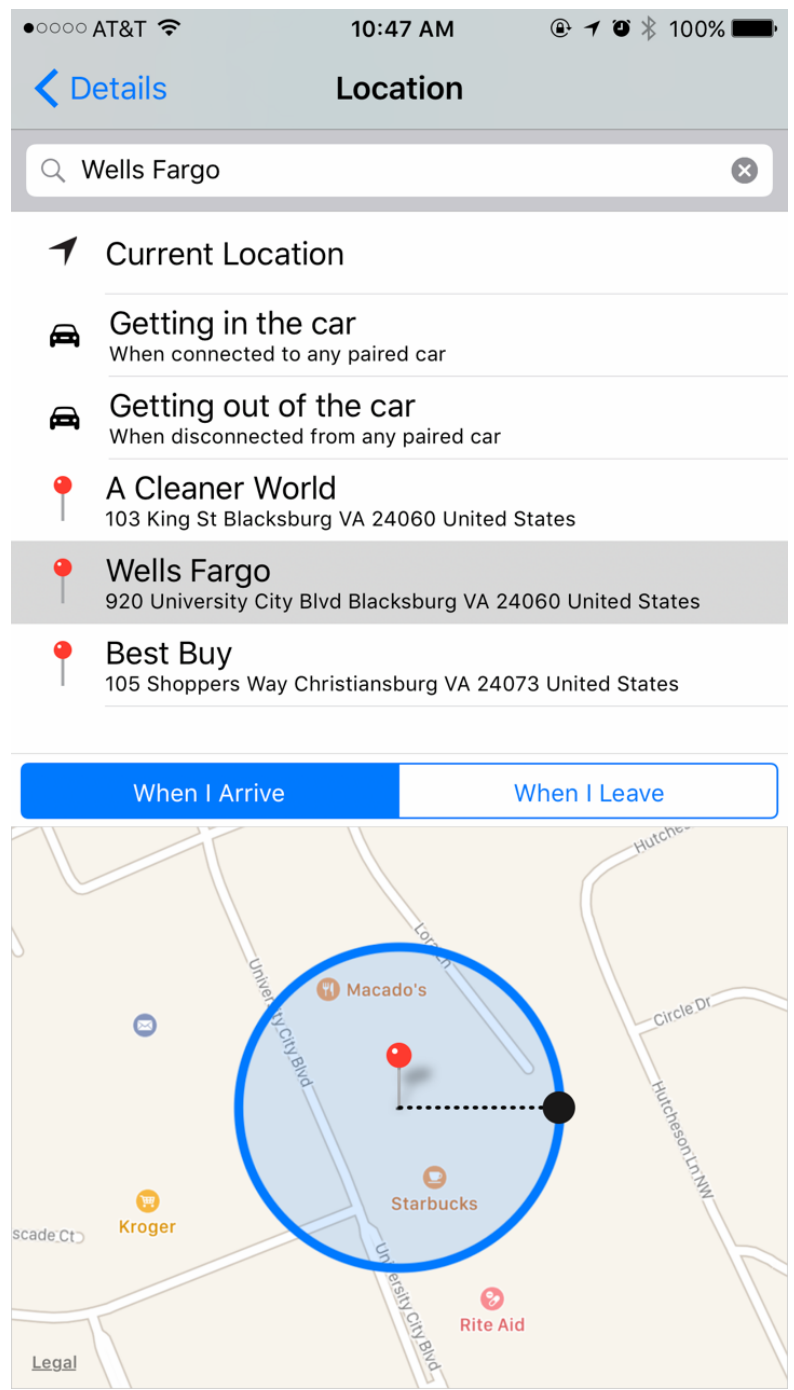

Figure 3.1: User interface in iOS Reminders showing how to setup up a location-based reminder 


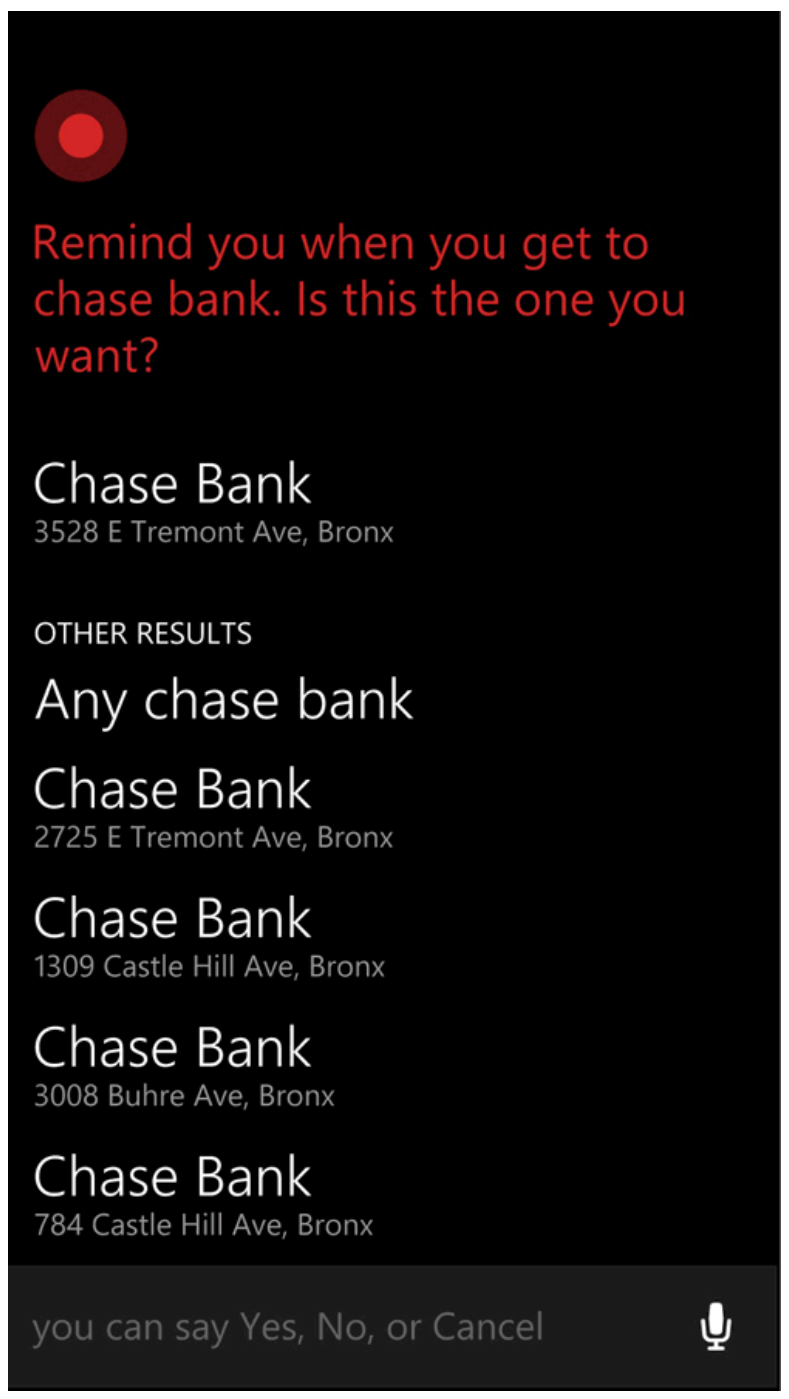

Figure 3.2: User interface of Windows Cortana showing how to set up a location-based reminder 


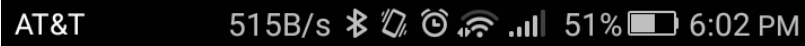

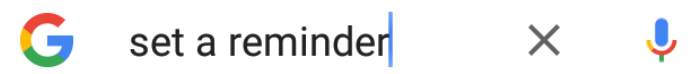

Add a reminder

Title

Get cash from wells fargo

Time Place

Location

Wells Fargo Bank

All locations
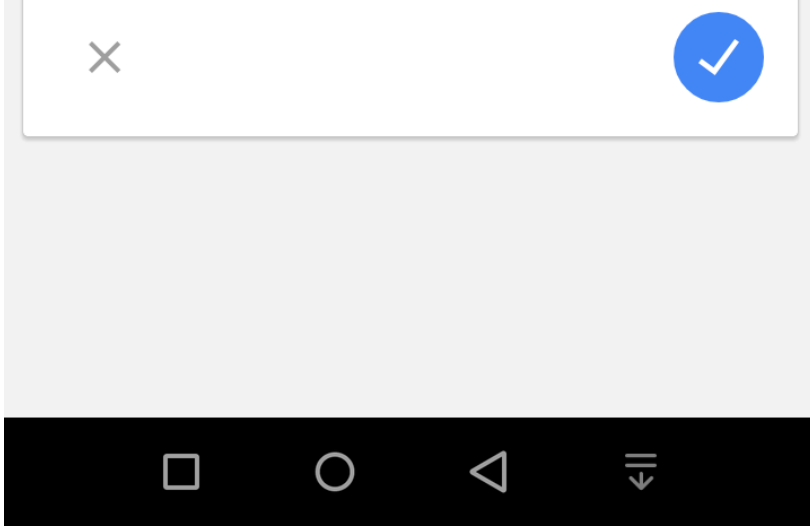

Figure 3.3: User interface of Google Now showing how to set up a location-based reminder 


\section{Chapter 4}

\section{Usability Study of Existing LBRs}

In this chapter, I report a short usability evaluation study for a commercial LBR application. This study aimed to understand how people use LBRs in a natural mobile environment and to uncover usability problems of the current interface design. The results show that the current design of LBRs fail to deliver the reminder effectively.

\subsection{User Study Setup}

I evaluated a list of available commercial LBRs, and selected LBR Checkmark based on high reviews in the online app store. I considered it to be a good design based on an expert heuristic evaluation using Nielsen's usability heuristics [Nielsen, 2005]. This reminder system supports both location-based and time-based reminders separately. However, this study only tested the usage of location-based reminders since I focus on the mobility nature of the phone. The LBR Checkmark allows users to set up a list of frequently visited locations, and then set up reminders associated with each location. A radius can be set when adding a location to define a kind of virtual associated area for that location. Departure mode and arrival mode 
can be selected when creating a reminder, to allow reminders popping up at arriving within the circle of the location radius, or upon leaving the circle. This system seems robust and easy to learn. A usability evaluation of LBR Checkmark on the iPhone was conducted in a natural testing environment, which involved a noisy background, lots of surrounding people, and unexpected interruptions and distractions.

\subsection{Study Procedure}

I recruited five participants (2 females and 3 males) through campus list server for this study. Each of the participants uses a mobile phone every day, but none of them had experiences using LBRs. The study consisted of two parts. In the first part, all participants were given a list of eight predefined tasks that needed to be done in different buildings on campus. The participants were asked to set up reminders using LBR Checkmark for each of the eight tasks. All these tasks were closely associated with students' daily activities, for example, "remember to return the book to library." Right after the participants had finished the setup process, I moved forward to the second part of the study. In the second part, the participants were asked to walk around campus to see how LBR Checkmark would trigger a reminder for the task they had entered. For example, the "return the book" reminder would pop up while the participants were approaching the library. The participants were given a predefined route on campus and were asked to follow the route, so they would pass by each of the specified locations. I followed the participants to capture direct observations. Think-aloud protocol and questionnaires were used to collect data. 


\begin{tabular}{rc}
\hline Usability Issue Categories & Total Number of Issues \\
User Interface Design & 8 \\
Mobile System Design & 10 \\
Lacking Context-awareness & 5 \\
User's Spatial Cognition & 4 \\
\hline
\end{tabular}

Table 4.1: Usability Issue Categories

\subsection{Usability Issues}

This study uncovered a total of twenty-seven usability problems with LBR Checkmark. These usability issues can be categorized into four aspects: user interface design problems, system design problems, context-awareness problems and user's spatial cognition problems.

The eight user interface design problems were related to the icon design, the image usage and so on. For example, all of the five participants ignored the bottom radius-setting bar at the first time. Compared with the bottom white text, the colorful map in the middle is more salient and attracts more attention. Also, the timer icon is designed similarly to the location icon, which introduced a source of confusion.

The ten system design issues are because of the complex system structure. For example, users were asked to add the location first and then create the reminders related to that particular location. However, two participants thought they were done after setting up the location and did not recognize that they had a few more steps to create a reminder. None of the participants had ever used this kind of system before. They were accustomed to recording a to-do task first, and then further add the attributes such as time and/or location to that task. The mental model of this LBR X did not match users' mental models, so the system could not function as expected. The study results showed that most problems with the reminder system design require a streamlined setup, such as writing something down quickly on a piece of paper. 
Meanwhile, the other two categories of problems were more mobile-related. Most of the context-awareness issues accounted for the failure of detecting the necessary context. The context surrounding the users changes all the time, which also influences users' satisfactions of using the reminder system. For example, four participants complained that when multiple reminders popped up at the same time, they had no idea which task to do first. Therefore, detecting users' moving patterns - such as remaining static, walking, or driving - could help the system deliver the reminders more appropriately and effectively. Interestingly, one participant was interrupted by a friend while he was walking around the campus. In the meantime, a reminder popped up. He saw the reminder and then kept chatting with his friends. While he was standing still, the same reminder kept alerting him, which annoyed him. Since the location detection problem is more related to physical limitations, such as the accuracy limitation of GPS, it is important for context-aware mobile reminders to consider these limitations and to try and employ users' current situations.

The other four problems were associated with how people understand locations. For instance, one participant did not understand how to set a radius, as he perceived location as a point rather than an area. The user considered the target location, a building, as a point. The participant expected the reminder to pop up only if he was inside the building, but definitely not when leaving or arriving. Another problem I found is that users' perceived distance differed from actual distance. Three participants were satisfied with the radius as 640 feet (which is the default value for the LBR X app) when they were preparing a reminder. Later, when they walked around the campus and received the reminder, they were surprised, with one of them expressing, "Woo, 640 feet is that far." Users, without any training in geography [Hirtle, 2011], often cannot accurately tell how far 1,000 feet is. However, they can tell how many blocks it is to a location or how many minutes walking it takes to reach a destination. LBR X reminded one participant of a to-do appropriately given that he was within the 
"geofence" of the target location. Unfortunately, he was actually quite far away from the door to the building. This result emphasized the deficiency of using "geofencing" without considering human moving patterns and the geographical layout of the destination. This problem was first discovered by [Ludford et al., 2006], but it is still not addressed in most of today's LBRs. Another interesting problem is that three participants did not understand how to input a location. They assumed that they had to be at the specific location first, and then set up the reminder by importing the current location details, because they couldn't find the exact location on the map. People differ in their abilities when it comes to reading maps. When people fail to input the address in the search bar, the map should be able to present different modes of spatial information for users to perceive the location, such as a street view or a text description.

\subsection{Considering Human Spatial Cognition}

LBR system design should focus particularly on users' spatial cognition and location perception. For example, user-perceived distance is different from the actual distance [Hirtle and Frank, 1997]. When users set the reminder radius without moving, usually they cannot correctly perceive the distance. Most people cannot correctly tell how far 1,000 feet is, whereas they can simply tell how far a block or a plaza is. The distance perception in users' mental models is quite different both from the actual and from each other. Allowing users to select a reminder radius using physical metaphors should be more helpful. Also it is well known that space and time are tightly coupled [Hirtle and Frank, 1997]. When setting the appropriate radius, the current system only allows users to set up by meters or feet, which violates users' real world experiences. The system should also allow users to set five minutes' walk or two minutes' drive, based on their different situations. Lastly, due to the different abilities when read- 
ing maps, the system should provide different modes to present spatial information, such as paths, nodes, and landmarks. Naive geography framework [Hirtle and Frank, 1997] also pointed out that people use multiple conceptions of space: one can think of locations as both points and areas. It is for that reason one participant failed to set the radius, since she perceived the location as a point. Human spatial cognition also seems to operate differently in small-scale spaces than in large-scale spaces [Hirtle, 2011], so the system design should take care of this aspect to avoid mixing two perceptions together.

\subsection{Summary}

This study demonstrated the inadequacy of "geofencing," as it fails to consider how people perceive and use location information in remembering their daily location-based to-do tasks. This chapter opens ideas for future LBR designs by considering human memory and spatial knowledge. 


\section{Chapter 5}

\section{Classification of Location-based To-do}

\section{Tasks}

In this chapter, I discuss a survey I conducted to collect typical to-do tasks that include location information. The findings suggest that location-based to-do tasks are more complicated than what existing LBRs support. Based on the results, I propose a classification of different uses of locations in location-based to-do tasks. The different uses of locations, as per my classification, have implications for the design of future LBRs.

\subsection{Online Survey}

I set up an online survey to collect to-do tasks from users. This survey aimed to understand how people use locations when they are organizing a to-do list. I announced and distributed the online survey through several mailing lists, seeking a broad group of participants. I collected to-do tasks from 129 respondents, both from industry and academia. Participants age ranged from 18 to 57 years old $($ mean=30). The majority of respondents were graduate 
students (69 \%); faculty members and undergraduate students accounted for $13 \%$ and 10\%, respectively. Ten percent of the respondents were from industry. Each participant reported from 1 to 5 tasks that they needed to remember to do in a few days, and on average each participant provided 3.2 tasks. Tasks such as "plan to start my business" or "to have a family" were considered long-term plans, and thus were removed from the responses. After excluding these long-term plans, 398 to-do tasks were left. Each task was classified based on how locations were specified in the task. I classified tasks like "submit homework" and "call Mom" as not associated with a location since these can be completed in any place. After accounting for these location-agnostic tasks, 181 of the 398 tasks were selected as being associated with a location.

\subsection{Data Analysis}

From the remaining 181 tasks, I identified three types of locations. First, 87 (48\%) tasks had to be completed at a specific location. Thirteen of them included the location in the task itself. For example, "visit a research dept at X Hospital" and "pick up something at CVS" both mentioned a specific location where to accomplish the task. This type of task is analogous to the task@place reported by Sohn et al. [Sohn et al., 2005]. The remaining 74 tasks did not specify a location explicitly, but in some way they required a particular place to accomplish the task. For example, tasks such as "pick up mail" (post office) and "take out garbage" (home) implicitly specified the location. This type of task is similar to the tasks in the category of "bring/get an item from a place" reported by Sohn et al. [Sohn et al., 2005]. However, their study only described one use of location, which is the task to be completed at a particular location. My work has expanded the description of uses of locations to three types. 
I identified a second type of location from the to-do corpus. I found that $86(47.6 \%)$ tasks specified a location type, but not the specific location. In this group, the task can be done at any one of several possible locations. For example, "get gas for the car" can be done at any gas station. People in the survey mentioned this type of task without specifying which gas station. It is a location-based to-do task that needs to be done in a location with gas service, but it does not specify any particular gas station.

The third group in the to-do collection had just eight (4.4\%) tasks. This type of to-do required multiple activities at different locations to accomplish the task. For example, "return a book to the library" includes two separate activities at two locations. One activity is "to get the book" from its current resting place (e.g., home) and the other activity is "to drop off the book" when near the library. This is a more complex task and often involves delivery of objects from one location to the other. The multiple steps typically have an order to them (e.g., you must get the book before you drop it off). The second task is dependent on the completion of the first. If the first task is not done yet, the second one is impossible to do.

\subsection{Classification of Uses of Locations}

Based on the findings, I identified shortcomings of the use of "geofencing" in LBRs. The preliminary findings led me to conclude that there are different uses of location in to-do tasks and that these differences have an impact on the design of LBRs. Based on these differences, I propose the beginnings of a taxonomy of to-do tasks specifically for LBRs use. Table 5.1 lists the three types of locations I named. The rest of this section discusses these three types in some detail and how they interact with the "geofencing" model that is typical of LBRs. 


\subsubsection{Do Task $\mathrm{X}$ at Location A}

This type of task is the most typical location-based task, and usually involves doing a task at a location. In some cases, the location is specific (e.g., a particular pharmacy), and in other cases the location can be a generic type or category of location (e.g., grocery store). For example, a task such as "drop letter in a mailbox" can be done at any mailbox. Today's LBRs are perfectly suited for reminding people to complete the task at a specific location, but do not allow users to specify a generic location. For users, the generic version of a task must be converted to a specific location first. The user must decide on a suitable location (e.g., plan where to get gas) and then enter the task as a specific version of the task (e.g., get gas at the station near work). It seems possible that future LBRs could offer dynamic suggestions for some common generic types of location (e.g., gas stations, mailboxes, grocery stores, etc.). I propose and evaluate an initial design for this type of task in chapter 9.

\subsubsection{Deliver $\mathrm{X}$ from $\mathrm{A}$ to $\mathrm{B}$}

This type of task is the second-most common task in the findings. The complexity for this type is that it involves two sequential tasks, picking up $\mathrm{X}$ from $\mathrm{A}$ and delivering $\mathrm{X}$ to B. In such cases, people have two location-based reminders rather than one. The second one is conditional upon the first one. If the user forgets to do the first part, the second one is irrelevant. Today's LBR software does not provide support for this complexity and moves the specification and management of these to-do tasks to the user. This type of task becomes more complicated when you consider that location A and/or B can also be generic, as discussed in the previous example. 
Table 5.1: Uses of location in to-do tasks

Do task X at location $\mathrm{A}$

Specific: drop paycheck at the rental office

Generic: drop postcard in a mailbox

Deliver X from A to B

Specific: bring book from home to office

Generic: take can goods to post-office

\begin{tabular}{l}
\hline \hline Go to location A \\
\hline \hline Specific: go to the library \\
Generic: go to a grocery store \\
\hline
\end{tabular}

\subsubsection{Go to Location A}

The last type of task is less common but nevertheless plausible. This is also problematic for a "geofencing" model. This type of reminder is for actually going to a particular location. Under "geofencing" the reminder will come up only when the user is near the target location. In fact, this kind of reminder might need to be triggered further out rather than upon arrival.

Proposing the taxonomy here is not claiming that this is an exhaustive classification, but it is a starting point to discuss how future LBRs can be designed and improved upon. The point of this study simply provides an idea to understand how people complete tasks at different locations in their real life, and to provoke the discussion to address the complexity of mobile tasks. 


\subsection{Summary}

This chapter proposed a classification of location use for to-do tasks based on the data collected for a survey. Designers of LBRs should explore how to use a more diverse set of types of locations in their applications. I believe that each type of location might require different interaction techniques to enter the location. Furthermore, I believe that the trigger of the reminders might be more nuanced than just a radial distance to a location. The proposed classification of uses of location helps discuss how future LBRs can be designed and improved. 


\section{Chapter 6}

\section{Framework}

This chapter provides a theoretical framework on how to make LBRs better by using theories of memory, in particular prospective memory, and treating the system that captures the LBRs as an external memory aid. With the knowledge from prospective memory, I set out to explore how to influence the design and the use of LBRs. I propose a theoretical framework that uses knowledge and principles from prospective memory, and present how LBRs might be improved. The ultimate goal is to facilitate human memory recall for prospective tasks.

The task flow for prospective memory activities discussed previously in Section 2.1 is mirrored in the task flow of LBRs. Figure 6.1 shows how I connect the prospective memory task flow with the LBR's task flow. To successfully finish a prospective memory task, LBRs play different roles in supporting human memory. By "supporting," I mean LBRs are considered as human external memory, which helps people record, store, retrieve, and maintain the information accordingly.

In the first stage, intention formation, LBRs serve as a memory-encoding aid. When people form an intention, they need to manually set up a to-do task on their LBRs. At the time 


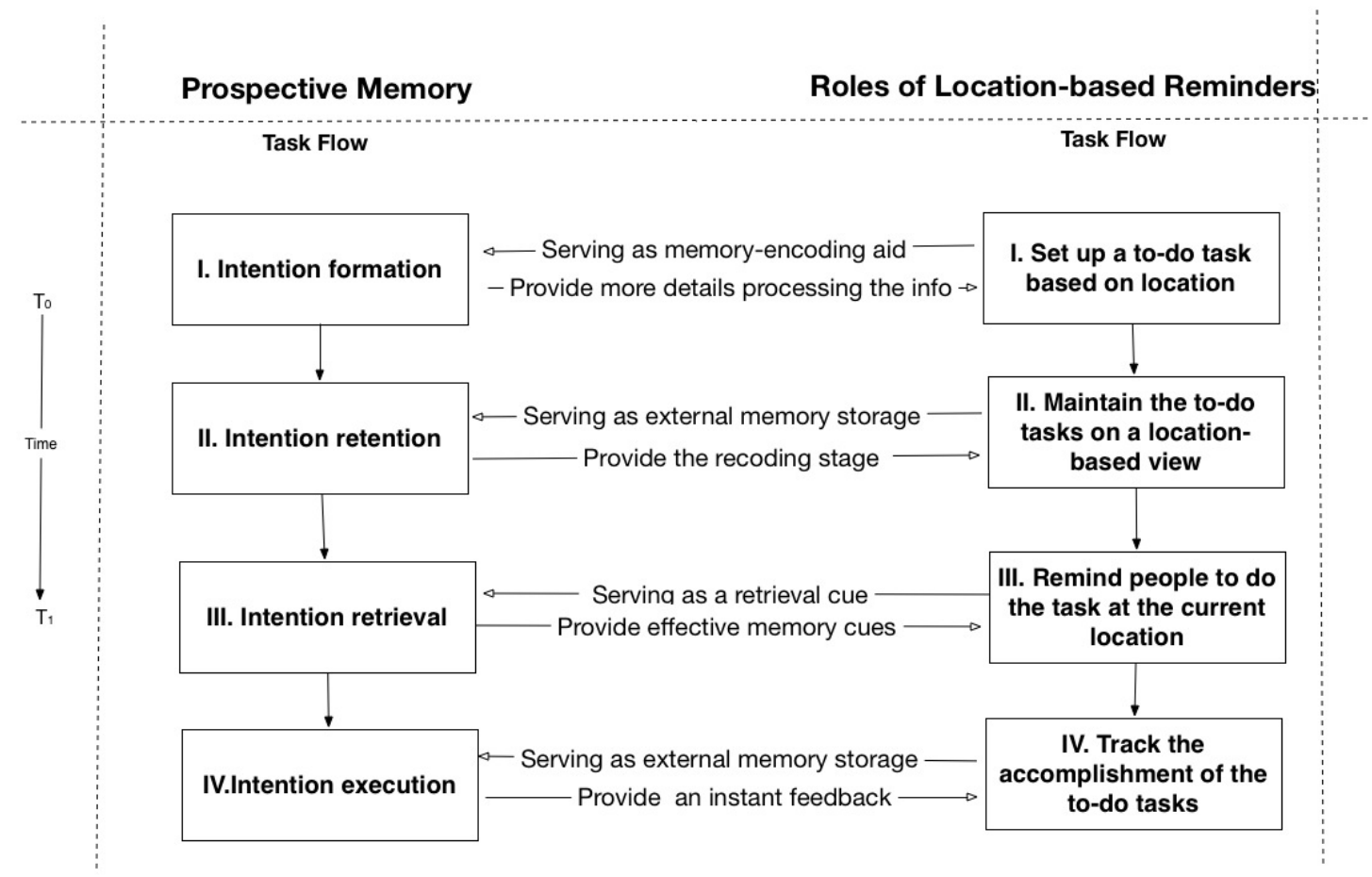

Figure 6.1: Theoretical Framework: prospective memory connected with LBRs

people input the intended task, they need to perceive and process the task into their working memory. This process involves attention resources, so when this piece of to-do task pops up at a later time, people are aware of it and can reconstruct the context for the action. As this process becomes automatic (e.g., email programs automatically adding reminders in calendar programs), fewer details are given to people when they are encoding. The result is that people have very fuzzy memories about the encoded task. The unintended result is that people rely more heavily on the external reminders rather than on their own memory.

In the second stage, intention retention, LBRs serve as external memory storage, similar to what a to-do list does. It provides people with a method to rehearse the to- do tasks by maintaining all of the information externally. More than a to-do list, tasks can be organized on a map-based view, which exploits human spatial memory to support remembering for location-based to-do tasks. The third stage is the time to perform the intended task, and the 
LBRs serve as an external retrieval cue. A salient cue that exists in the physical environment could work well in triggering the intended task, as long as it catches people's attention. A reminder sent by LBRs works like a salient cue. If the reminder is effective enough, it would interrupt people's ongoing activity and get people's attention to switch to the intended to-do task. Current LBR research prototypes mostly focus on improving this retrieval stage, trying to find a more effective location point to deliver the reminder.

Rather than having LBRs use one cue (one target location serving as one external cue), my work aims to provide more external cues in the encoding stage. These cues would come from the real-life environment at the moment of retrieving stage. For example, if the LBR system has an address or name of a location, such as a supermarket, the LBR can display such landmarks or a street view route at the time of encoding. I believe that this will increase the salience of the cue at the retrieval stage and thus improve the role of the memory aid for prospective memory tasks.

Finally, people need to execute the intended to-do task, and LBRs again serve as external memory storage by tracking the completed tasks. In addition, at the time of execution, people are often reminded of tasks to be done without the context of where the task was created. I will explore how I can reconstitute the context of the task at the execution time I am interested in how to improve the prospective remembering of daily activities. I focus the research on how external memory cues in the natural physical environment can improve the recollection of to-do tasks. Information captured in human memory sometimes cannot be used effectively because the information has not reached the awareness level. The stored information just needs an appropriate trigger to activate the memory, so it can be brought into the awareness level and catch people's attention.

In this chapter, I presented a framework that applies theories of prospective memory into 
the task flow supported by LBRs. Research in cognitive psychology has created a deep understanding of prospective memory. I explored how some theories can enhance human memory recall for prospective memory uses. I study the effectiveness of memory cues in this context during the task encoding process. My work takes advantage of existing ubiquitous infrastructure to present memory cues from the environment around us. To extend LBRs to a memory-encoding aid, the LBRs could automatically provide relevant contextual cues at the encoding stage. It should not require more human effort to input the information, as I feel that this can be done automatically using information available in today's ubiquitous environment. Furthermore, I feel that the extra cues in the encoding stage would require less human attention to process the provided cues at retrieval stage with the hypothesized result that these cues will further facilitate the task recall. 


\section{Chapter 7}

\section{The Match Between Encoding and Retrieval}

This chapter describes an empirical user study of how people remember and recall daily location-based to-do tasks. This study had two experimental sessions: the second session was scheduled three days after the first. In the first session, participants were given a set of eight daily to-do tasks to remember. In the second session, participants were asked to come back to recall the tasks they remembered from the first session. This study was approved by the Virginia Tech Institutional Review Board with approval \#14-198 on December 10, 2014.

\subsection{Experimental Design}

Most LBR systems show an overhead birds-eye view of the desired location at the encoding stage. This view is a poor match for the retrieval stage of a prospective to-do task. At retrieval time, users are typically walking or driving around and a first-person view might be a better cue for remembering a pending to-do task. Furthermore, the familiarity of the external 


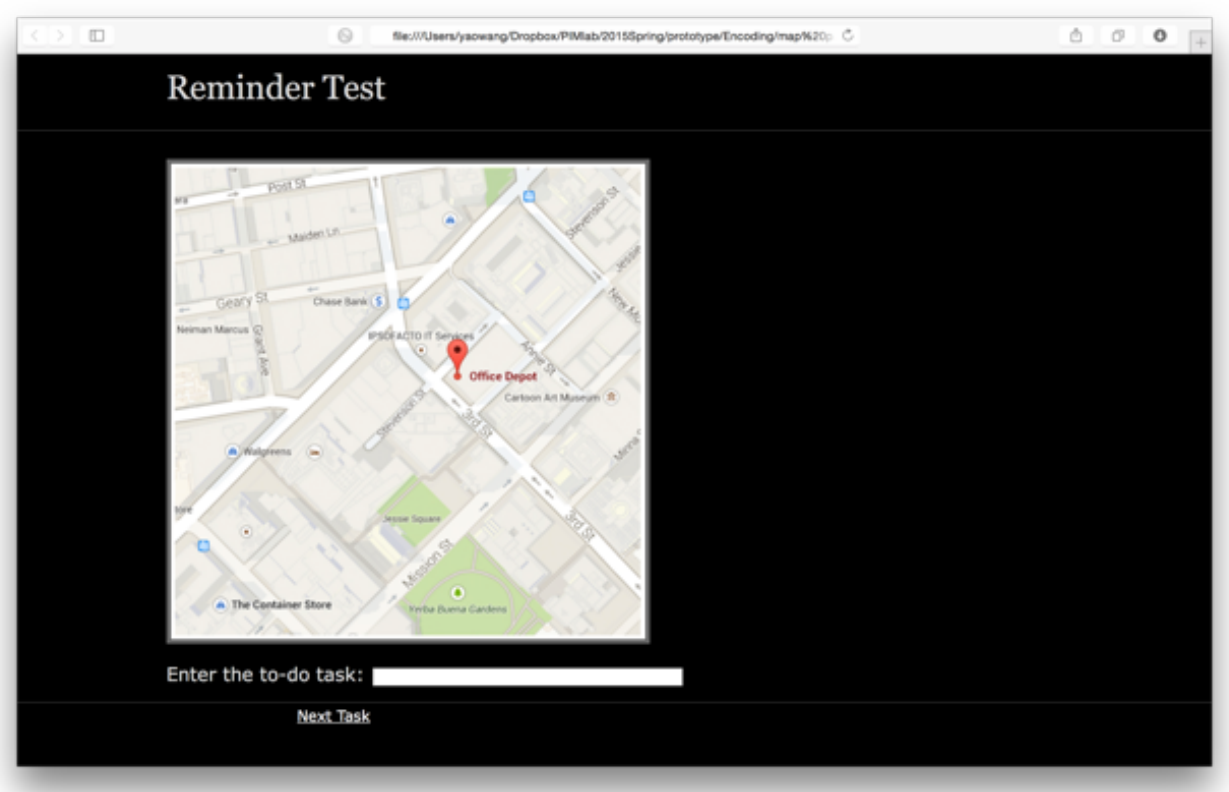

Figure 7.1: Map View interface for entering their to-do task, showing the Office Depot task. This is the view encountered in most commercial LBR systems.

cues (in my case the business logo and the business location) has been found to influence prospective memory [McDaniel and Einstein, 2000]. I am therefore also interested in that how the familiarity with the business and the location influences the recall performance.

I conducted a between-subject study with three groups, each seeing a different interface at the encoding stage. The study evaluates the impact that different interfaces at the encoding stage might have at the retrieval stage for a series of location-based to-do tasks. Furthermore, I explored what impact prior familiarity with the business location has on the recall performance.

The main research questions are:

- RQ1: Does encoding of a prospective to-do task with a first-person view produce better performance at the retrieval stage than encoding with a birds-eye view? 


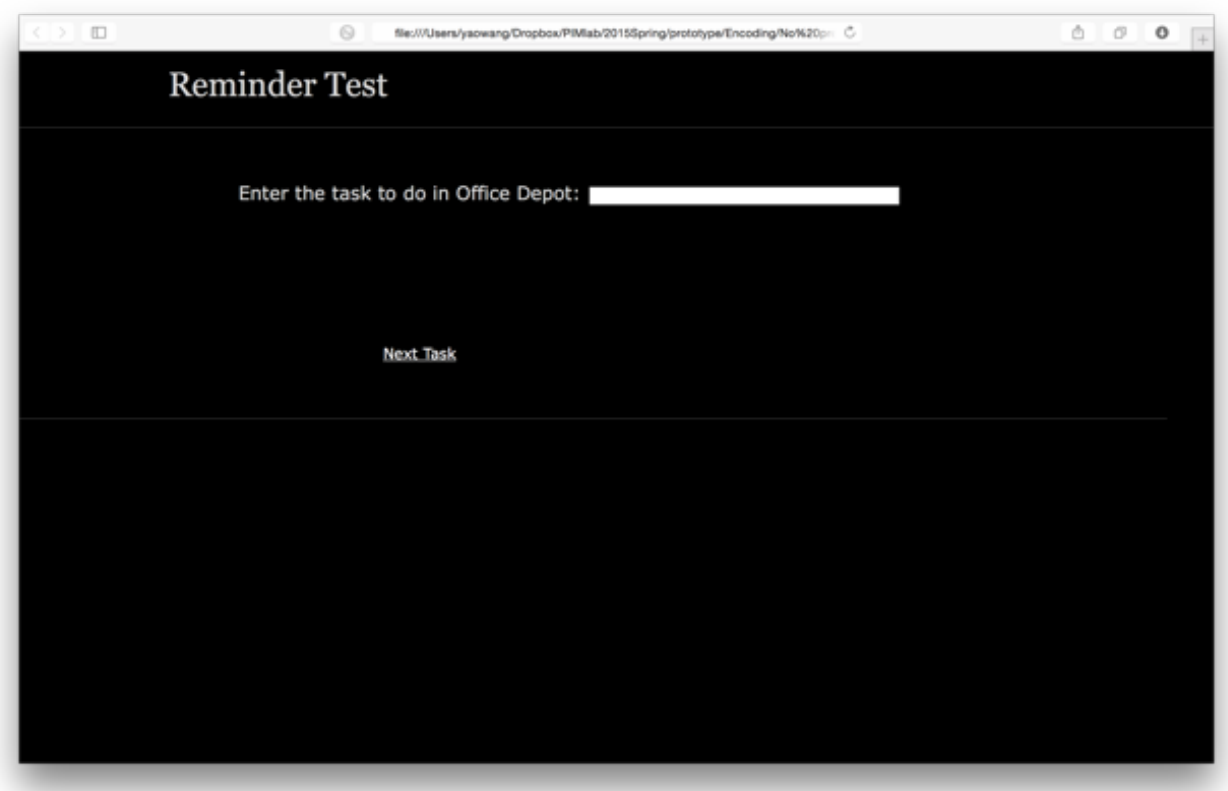

Figure 7.2: No Previous (control) interface for entering their to-do task, showing the Office Depot task.

- RQ2: What role does prior familiarity with a business name and logo have at the retrieval stage of a to-do task?

The independent variable is the encoding method, showing three groups: Street View, Map View, and No Preview. The dependent variables are different measures of performance (total recall, location recognition, task recall), effort (encoding and retrieval), familiarity with the business logo, and other personal attributes (gender, memory ability, and education).

Street View Group. Participants in this group saw a Google Street view (e.g., a first-person point of view) of the business location (see Figure 8.2) at the encoding stage.

Map View Group. Participants in this group saw a Google Map view (e.g., birds-eye view) of the business location marked on a map (see Figure 8.1) at the encoding stage.

No Preview (Control) Group. Participants in this group did not see any image at the encoding 


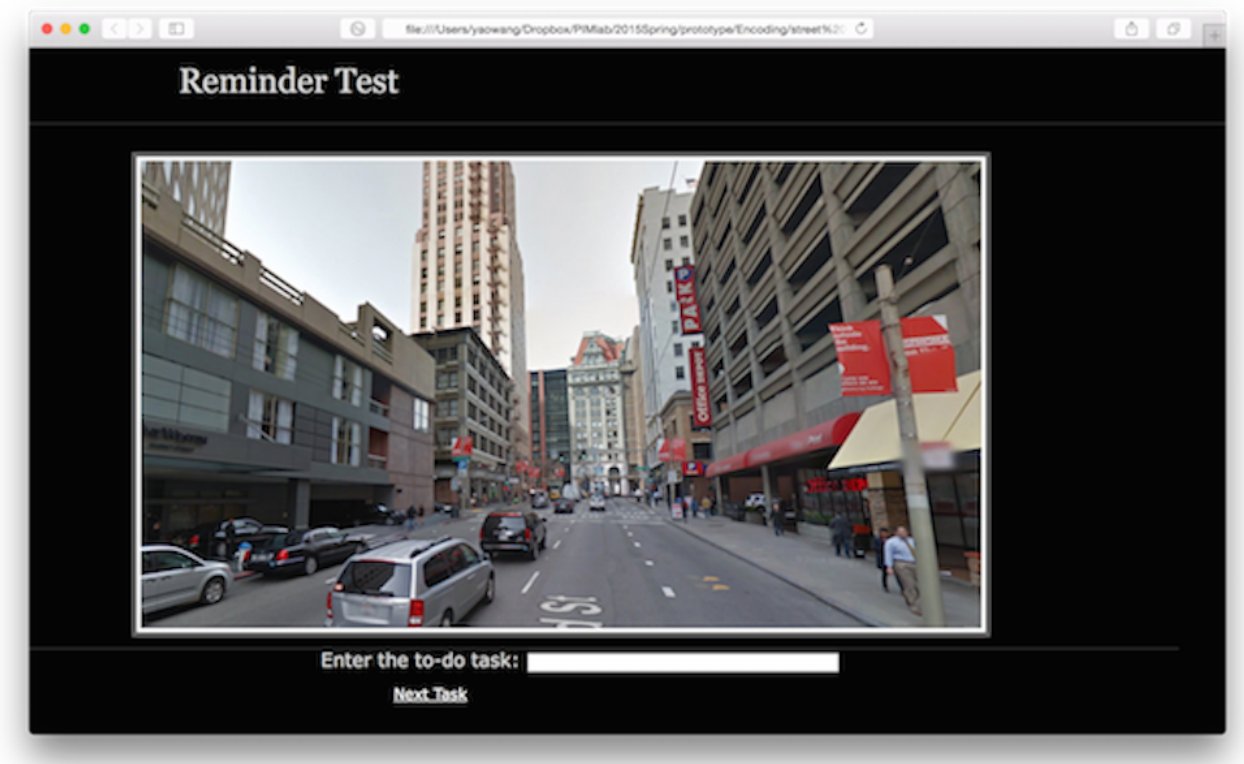

Figure 7.3: Street View interface for entering their to-do task, showing the Office Depot task.

stage (see Figure 7.2). The screen displayed only the text input field to type the to-do task. To make the three conditions similar, I displayed the location name (in text) in the prompt for the text input field. This group served as a control group.

\subsection{Study Setup}

\subsubsection{Participants}

I recruited participants by announcing the study in the SONA Experiment Management System used by the Psychology Department, as well as posting an announcement in several listservs on campus. Sixty participants participated, either voluntarily or for course credit. Participants were between 18 and 34 years of age. There were 43 female participants and 17 male participants. The participants' backgrounds covered a variety of majors from hu- 


\begin{tabular}{|c|c|c|c|}
\hline & No Preview & Map View & Street View \\
\hline $\begin{array}{l}\text { Num. Par- } \\
\text { ticipants }\end{array}$ & 20 & 20 & 20 \\
\hline Avg. Age & $22.35(18-33)$ & $22.85(18-34)$ & $22.3(18-29)$ \\
\hline Gender & 17 females and 3 males & 11 females and 9 males & 15 females and 5 males \\
\hline Education & $\begin{array}{l}10 \text { undergraduates, } \\
\text { masters and } 6 \text { doctoral }\end{array}$ & $\begin{array}{l}10 \text { undergraduates, } 5 \\
\text { masters and } 5 \text { doctoral }\end{array}$ & $\begin{array}{l}8 \text { undergraduates, } 5 \text { mas- } \\
\text { ters, } 16 \text { doctoral and } 1 \text { no } \\
\text { answer }\end{array}$ \\
\hline $\begin{array}{l}\text { Memory } \\
\text { Ability }\end{array}$ & $\begin{array}{l}9 \text { (bad memory) and } 11 \\
\text { (good memory) }\end{array}$ & $\begin{array}{l}10 \text { (bad memory) and } 10 \\
\text { (good memory) }\end{array}$ & $\begin{array}{l}15 \text { (bad memory) and } 5 \\
\text { (good memory) }\end{array}$ \\
\hline $\begin{array}{l}\text { Familiarity } \\
\text { with the } \\
\text { city }\end{array}$ & $\begin{array}{l}7 \text { (travelled once/twice) } \\
\text { and } 13 \text { (never) }\end{array}$ & $\begin{array}{l}3 \text { (travelled once/twice) } \\
\text { and } 17 \text { (never) }\end{array}$ & $\begin{array}{l}6 \text { (travelled once/twice), } \\
1 \text { (travelled } 10 \text { times) and } \\
13 \text { (never) }\end{array}$ \\
\hline
\end{tabular}

Table 7.1: Participants' background information

manities, STEM and medical fields. Forty-three of the participants had never been to San Francisco before; 16 participants had traveled there once or twice; and only 1 participant had traveled there more than 10 times.

Table 8.1 shows a summary of the participants' background information.

\subsubsection{Procedure}

I conducted the study in a closed room typically used for usability studies. The room was equipped with a desk and chair. The study consisted of two sessions, with the second session taking place three days after the first. Each session took approximately 30 minutes. In the first session (encoding stage), I asked participants to memorize a list of eight to-do tasks, each with a predefined location. The interface for the experiment allowed the participants to enter whatever they found useful for remembering the task three days later. Prior their arrival, I assigned each participant into one of the three experimental conditions. All participants saw the same tasks, but the interface to enter the task was dictated by the experimental group. 
During the second session (retrieval stage), I asked participants to recognize the locations and recall the tasks they memorized in the first session. All the participants saw the same interface in this session.

\section{First Session: Encoding Stage}

Upon arrival, each participant filled out a consent form and completed a background questionnaire. The questionnaire included a self-assessment of their memory abilities which asked participants to rate from never to always as to how often these situations occurred: "forget an appointment," "forget to pay a bill," " forget to pick up or buy something at a particular place," "forget to bring something with you," or "forget to return something or drop something at a particular place." The questions were based on those used by Elsweiler et al. [Elsweiler et al., 2007].

Next, I presented the participants with instructions and the eight predefined task scenarios. The instructions for the first session were: Imagine it is 10am on Friday; you are at work now. You want to clean up the tasks that you need to complete later, so you start to prepare a to-do list with the tasks listed below. You need to enter all these to-do tasks into the computer. I asked participants to enter each task into the computer as if they were adding it to their real to-do task manager. Between tasks, I included the statement: Once you have finished remembering this task, you can click the "next task" button to continue. Participants were allowed to spend as long as they needed to enter each task. Once they were done, they would click the "next task" button. After they had encoded all the eight tasks, I conducted a short debriefing session as a wrap-up of Session One and as a way to remind them about the visit three days later for Session Two.

Each task used specified a particular location where they were to complete the task. All par- 
ticipants received the exact same task scenarios in the same sequence. The eight predefined task scenarios are listed below:

1. You ordered a wireless printer online, but shipping is expensive. You decided to ship it to the Office Depot for free. It has arrived, and you want to remember to pick up the printer while you are driving by the Office Depot in downtown.

2. Your friend Tom borrowed a book from the library at the Academy of Art University. He is out of town now and the due date of the book is today. AAU is near your workplace, so he asked you to return the book for him. You need to remember to return the book this afternoon.

3. Your roommate helped you solve a big problem in your homework yesterday. Peets Coffee $\&$ Tea was offering limited holiday specials. As a thank you, you would like to stop by Peets Coffee \& Tea to buy a holiday gift for your roommate.

4. You live in an apartment, and you need some quarters to use the washer and dryer in the basement. There is a Wells Fargo bank near the workplace, so you want to get some quarters at that Wells Fargo location.

5. The fall season is coming. You are having seasonal allergies. You would like to stop by Walgreens to pick up the allergies prescription drugs.

6. Your mother's birthday is coming. You would like to mail a package to your Mom as a gift. You need to go to FedEx to drop off the package.

7. You received a promotional letter from Citibank, so you decided to open a new credit card at Citibank this afternoon. You need to apply in person before the promotion expires today. 
8. You are going to host a birthday party at your house this weekend. Target is on your way home, so you decided to stop at Target to buy groceries.

\section{Second Session: Retrieval Stage}

I asked participants to come back three days later for the second session. I set three days as the interval to avoid participants remembering the tasks too readily. This was also done as a realistic condition, as people often prepare to-do lists to be completed in the near future.

Session Two consisted of watching a video taken from a car driving in downtown San Francisco, and passing by all the encoded locations. I asked the participants to look for the locations memorized during Session One. Figure 7.4 shows a snapshot of the video they saw; it was recorded from a camera mounted on the dashboard of a car while driving through downtown and was made specifically for this study. The first-person view was intended to be a simulation of the scenario people would encounter when they are traveling the city looking for a location. The length of the video for Session Two was 10 minutes and 49 seconds. Before the second session began, I allowed participants to practice the task using a similar but shorter video (only 18 seconds).

If participants recognized a location, they were instructed to pause the video and say out loud the memorized task and the location. At the same time, I asked the participants to count the traffic lights they passed by. I provided a piece of paper and pen just in case they needed it to keep track of the traffic lights. The purpose of counting traffic lights was to allocate part of the participants' attentions to a dual task (counting traffic lights) and away from focusing on the memorized locations. Participants were told to report the task as soon as they saw the corresponding location on the video. At the end of the video, they were asked to report if there were any remaining tasks that were not identified. 
Participants filled out a questionnaire upon completion of Session Two. The questionnaire asked participants to rate how familiar they were with the businesses and their logos (e.g., Office Depot, Target) used in the tasks.

\subsubsection{Data Collection}

For both sessions, I captured the screen interactions of the participants and recorded the audio of their comments and interviews.

During the first session, I recorded from the video the time each participant spent on encoding each task (encoding effort). I measured the total time of encoding from the display of the first task until the participants clicked the "I am done" button at the end of the session.

During the second session, I collected their recall performance. I collected the task they recalled and the location where the task was recalled. In some cases, participants recognized a location (e.g., "I have to do something at this bank") but not the task. In other situations, participants reached the end of the video and remembered some tasks they had to do, but did not recognize the location. I also collected the participant's count of traffic lights (retrieval effort).

I collected the answers to questions in the debriefing session as well as the answers to the questionnaire given at the end of the study. Participants self-assessed their memory abilities and rated the familiarity level of each of the locations on a rating scale from 1 to 5 , where 1 means very unfamiliar and 5 means very familiar with this location. Participants were also asked to rate the frequency with which they performed the types of tasks used in the study, on a rating scale from 1 to 5 , where 1 means very infrequently and 5 means very frequently perform this task. 


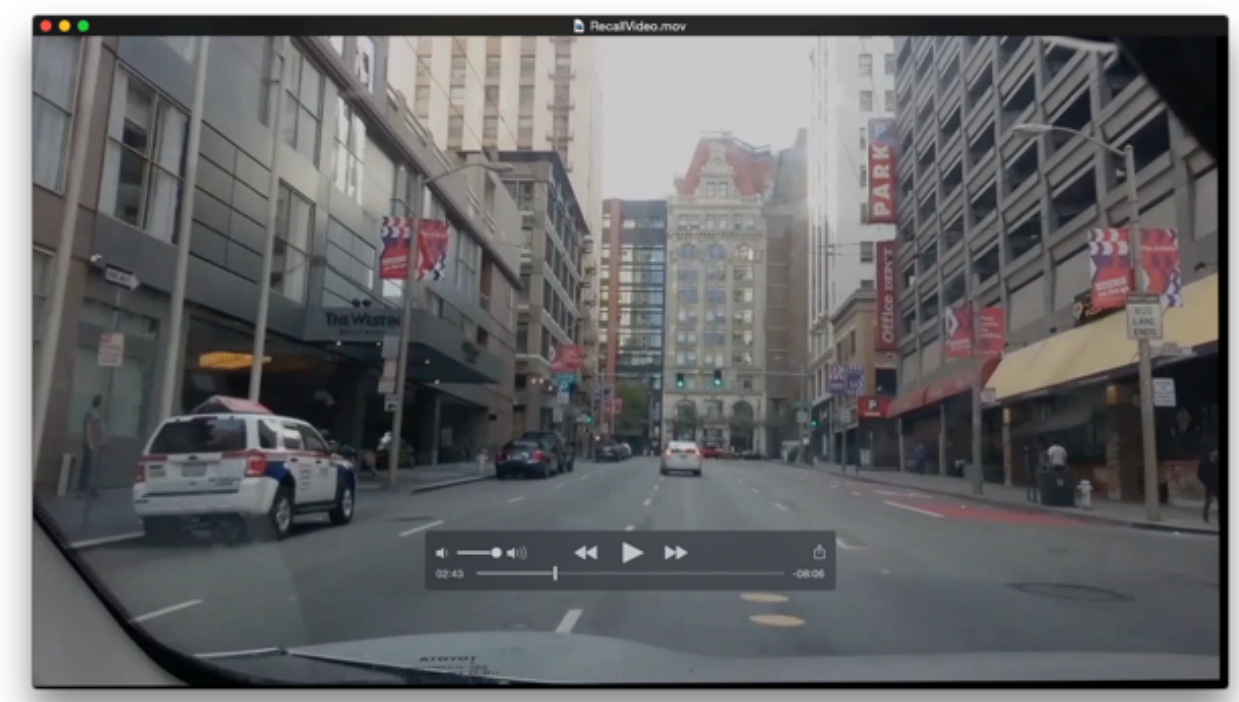

Figure 7.4: Interface for Session Two, showing the Office Depot task; all three groups used the same interface.

\subsection{Results}

The goal was to study the relationship between the interface at encoding stage for a prospective to-do task and the cues available in the real world at the point of doing the tasks.

\subsubsection{Complete Recall}

I consider a recall a Complete Recall if the participants recalled the task at the correct location. Some recognized a location but did not remember the task to be performed there. Other participants failed to identify the locations while watching the video, but remembered tasks at the end of the session. These two performance measures were counted separately from the complete recall performance.

Participants had a complete recall ranging from 0 (none) to 8 (recalled all pairs, perfect score). Two participants (one in the Control group and one in the Map View group) were 


\begin{tabular}{rccc}
\hline Title & No Preview & Map View & Street View \\
\hline Mean & 2.75 & 3.20 & 5.20 \\
SD & 1.71 & 2.17 & 2.09 \\
Min & 0.00 & 0.00 & 1.00 \\
Max & 6.00 & 7.00 & 8.00 \\
\hline
\end{tabular}

Table 7.2: Means and standard deviations of the number of Complete Recall in three groups. The scores ranged from 0 (all tasks wrong) to 8 (perfect score).

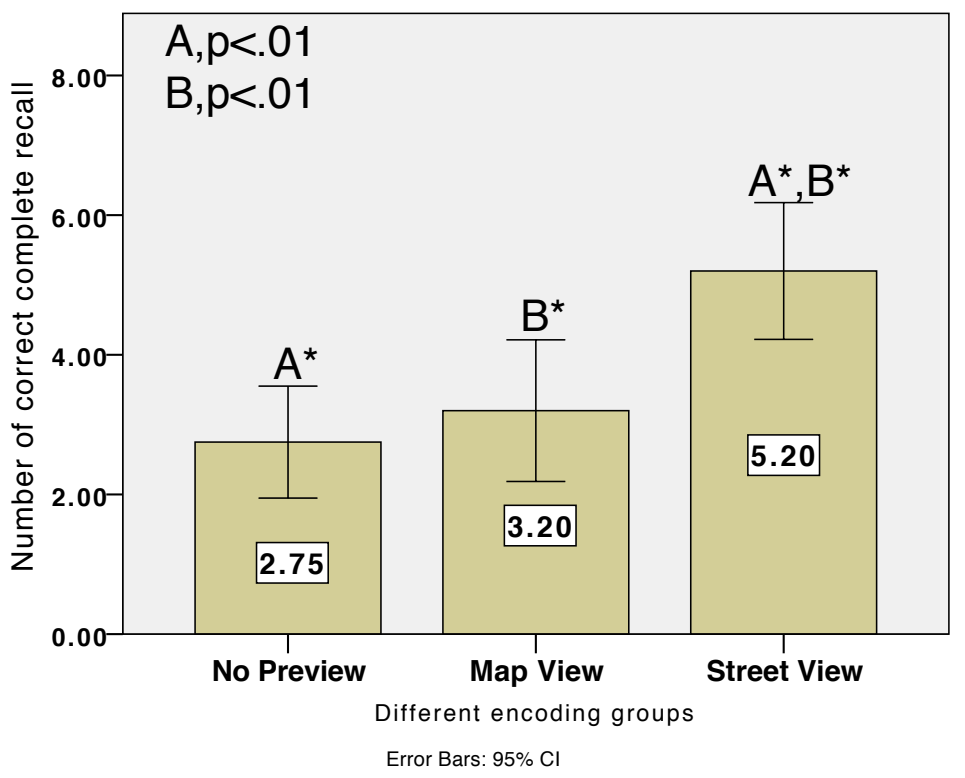

Figure 7.5: Complete Recall performance for all three groups. I found a significant difference between Street View and the other two groups

not able to correctly recall any correct pairs of location and task. Three participants (all in the Street View group) had a perfect complete recall. Table 7.2 shows the means and standard deviations for complete recall for the three groups.

I conducted a one-way between-subjects ANOVA to compare the effect of encoding match for prospective location-based tasks on complete recall performance in Street View, Map View, and No Preview (control) conditions. There was a significant effect of the interface used at encoding on the number of complete recalls at the $p<.05$ level for the three conditions $[F(2,57)=8.499, p=.001]$. Post hoc comparisons using the Tukey HSD (honest significant 


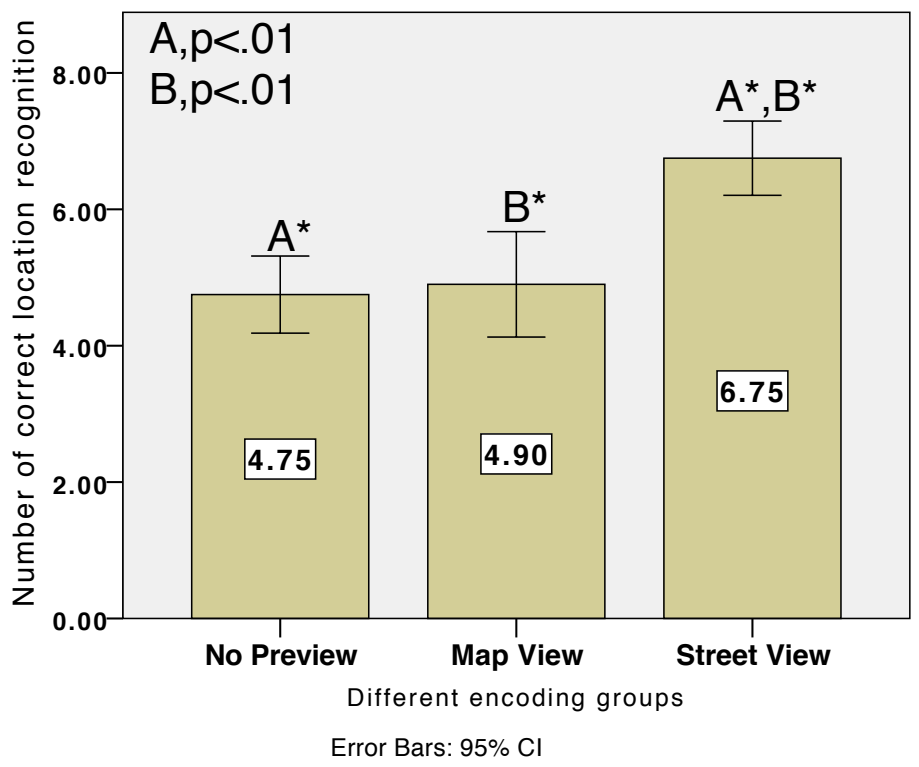

Figure 7.6: Location recognition for all three conditions

difference) test indicated that the mean score for the Street View $(M=5.20, S D=2.09)$ was significantly different than the No Preview $(M=2.75, S D=1.71)$, and also significantly different than the Map View $(M=3.20, S D=2.17)$. However, the Map View $(M=$ $3.20, S D=2.17)$ did not significantly differ from the No Preview $(M=2.75, S D=1.71)$. Figure 7.5 shows the results of this test.

\subsubsection{Location Recognition}

I use Location Recognition to represent participant's performance in recognizing the locations for the to-do tasks. This measure only includes recognition of the location, ignoring whether or not participants recalled the tasks associated with the location.

I conducted a one-way between-subjects ANOVA to compare the effect of encoding match for prospective location-based tasks on the number of locations recognized in Street View, Map View, and No Preview (control) conditions. There was a significant effect of the interface used 


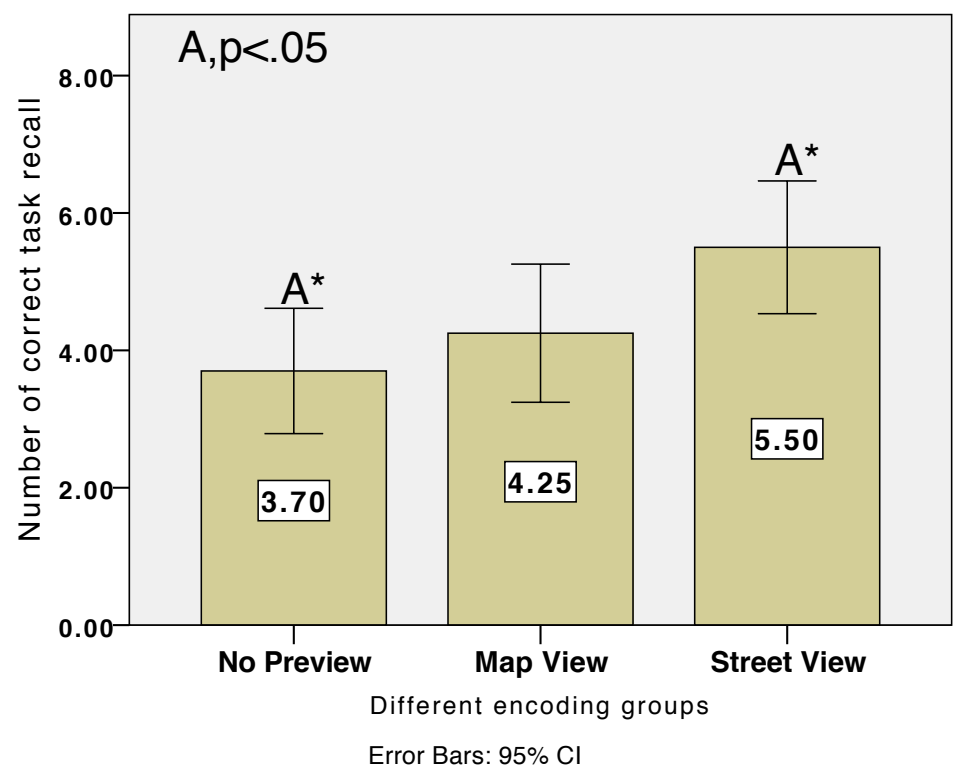

Figure 7.7: Task Recall for all three conditions

at encoding on the number of locations recognized at the $p<.05$ level for the three conditions $[F(2,57)=13.434, p=.000]$. Post hoc comparisons using the Tukey HSD (honest significant difference) test indicated that the mean score for the Street View $(M=6.75, S D=1.16)$ was significantly different than the No Preview $(M=4.75, S D=1.21)$, and significantly different than the Map View $(M=4.90, S D=1.65)$. However, the Map View $(M=4.90, S D=1.65)$ did not significantly differ from the No Preview $(M=4.75, S D=1.21)$. Figure 7.6 shows the results of this test.

\subsubsection{Task Recall}

I used the number of Task Recalls as another measure of performance. Task recall could happen together at the time of recognizing a location or it might happen at the end of Session Two when a participant commented, "oh, I was supposed to buy groceries but I did not see Target." 
I conducted a one-way between-subjects ANOVA to compare the effect of encoding match for prospective location-based tasks on the number of tasks recalled in Street View, Map View, and No Preview (control) conditions. There was a significant effect of the interface used at encoding on number of tasks recalled at the $p<.05$ level for the three conditions $[F(2,57)=4.026, p=.023]$. Post hoc comparisons using the Tukey HSD (honest significant difference) test indicated that the mean score for the Street View $(M=5.50, S D=2.06)$ was significantly different than the No Preview $(M=3.70, S D=1.95)$. However, the Map View $(M=4.25, S D=2.15)$ did not significantly differ from the No Preview $(M=3.70, S D=$ $1.95)$ and Street View $(M=5.50, S D=2.06)$. Figure 7.7 shows the results of this test.

\subsubsection{Encoding Effort}

To assess how much effort each participant spent on memorizing the tasks (encoding), I analyzed the time spent on Session One. I conducted a one-way between-subjects ANOVA to compare the time spent in encoding the tasks in Street View, Map View, and No Preview (control) conditions. There was no significant effect of the interface used at encoding on the amount of time spent on encoding at the $p<.05$ level for the three conditions $[F(2,57)=$ $.183, p=.834]$. The effort spent in memorizing the tasks was similar across all groups, eliminating the possibility of one group spending more time/effort memorizing tasks than the others.

\subsubsection{Retrieval Effort}

I instructed participants to count the traffic lights at the retrieval stage, so they would not put all the attentional resources on location recognition. At the end of the video, I asked each participant to report the total number of the traffic lights they found. There was a 


\begin{tabular}{rccc}
\hline & No Preview & Map View & Street View \\
\hline Mean & 27.85 & 26.90 & 27.25 \\
SD & 3.45 & 4.56 & 5.61 \\
Min & 20 & 19 & 9 \\
Max & 33 & 37 & 33 \\
\hline
\end{tabular}

Table 7.3: Means and standard deviations for traffic lights count among three groups

total of 33 traffic lights in the video. Table 7.3 shows the means and standard deviations for the count of traffic lights.

I conducted a one-way between-subjects ANOVA to compare the traffic light counts in Street View, Map View, and No Preview (control) conditions. There was no significant effect at retrieval time on the number of traffic lights counted at the $p<.05$ level for the three conditions $[F(2,57)=.216, p=.807]$.

However, note in Table 7.3, the Map View had a maximum count of 37, when the total of traffic lights was 33. Upon inspection, I realized that if the car in the video stopped at a red traffic light, one participant counted the traffic light a second time when it changed to green. There were 10 traffic lights that turned from red to green during the video used in the experiment. Nevertheless, there was no significant differences in the traffic light counts between groups, so I feel that this miscount did not unfairly bias one group over the other.

\subsubsection{The Impact of Familiarity}

I looked further into some particular tasks in the first study to ensure that previous familiarity with the businesses (or the logo) did not have an undue influence in the results. Since the university is located in Virginia, far from San Francisco, I felt I had a setup that reduced familiarity with the location. Nevertheless, participants might have familiarity with the businesses and their logos independent of the city. 


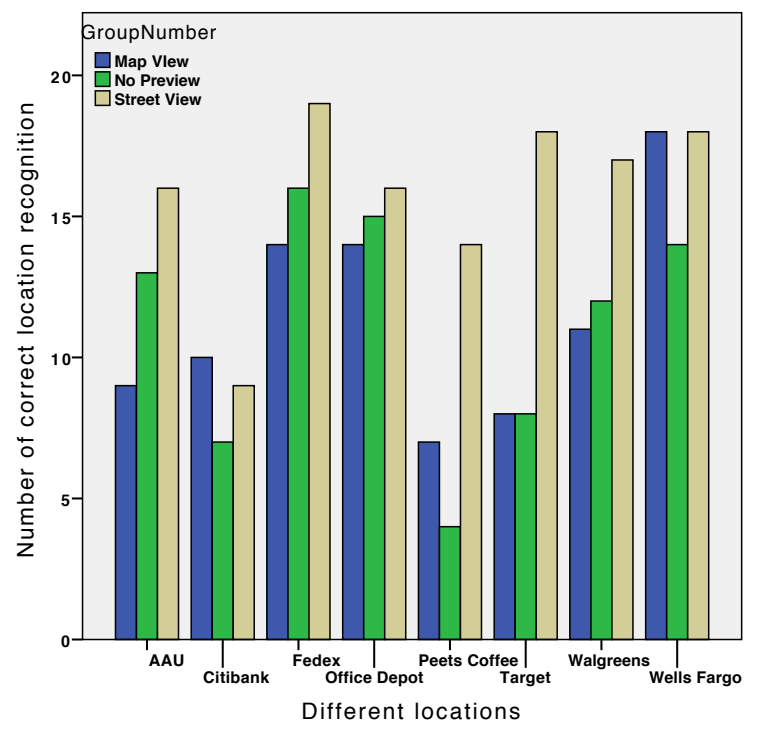

Figure 7.8: Location Recogition for all tasks in all three conditions

Figure 7.8 shows the averages for location recognition for all tasks performed in the experiment. As the statistical analysis already showed, the Street View group performed better than the other two groups. However, it is worth highlighting the gaps within each group for particular tasks. There is a large gap between groups for the Academy of Art University (AAU), Peets Coffee \& Tea, and Target. I explore these three tasks individually next.

First, Figure 7.9 shows the average familiarity level of each business rated by the participants. AAU scored the lowest number, making it the most unfamiliar place in the study. Fifty-one out of sixty participants had not heard of this institution; AAU is in San Francisco and local to that city. Peets Coffee \& Tea was the second most unfamiliar place; 38 of the participants had never heard of this business. While the brand Peets Coffee \& Tea is served in some restaurants in the area, there is no coffee shop for this franchise within 25 miles of the town (according to the listing of locations on their website).

On the other hand, however, is Target. Target was rated as the most familiar business and logo by the participants (average of 4.28 out of 5 , as shown in Figure 7.9). Yet, it had a 


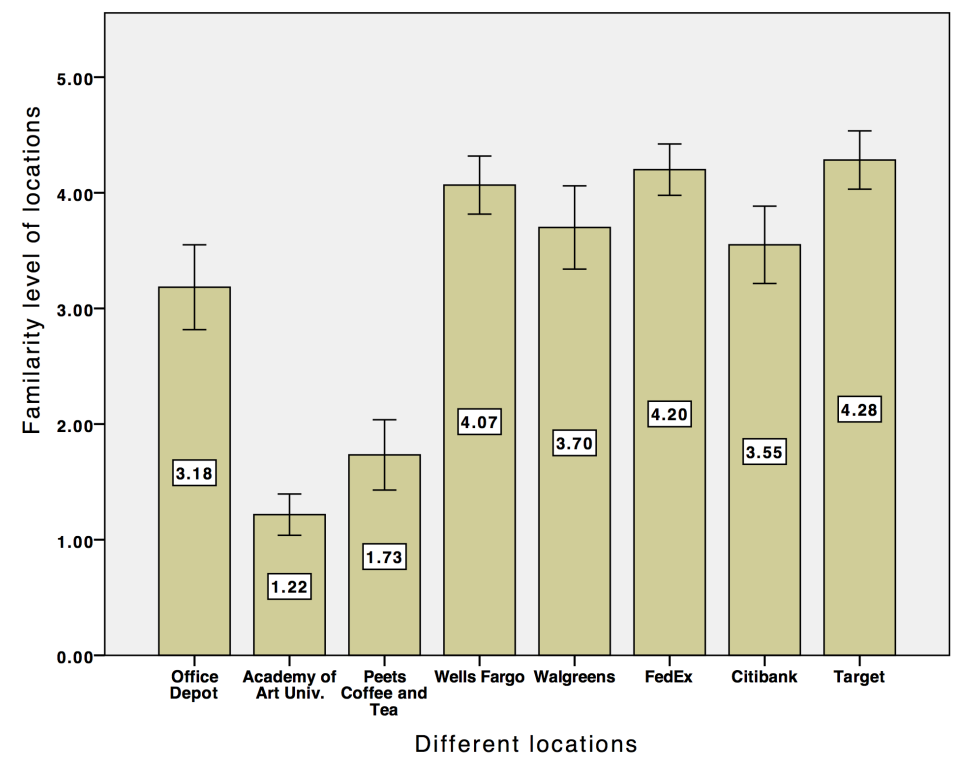

Figure 7.9: Familiarity level with the business or logo used in the study

low Location Recognition performance in the Map View group and the No Preview (control) group (see Figure 7.8). I consider this location to be a special case in the data set. Figure 7.10 shows a combined image of the Target in San Francisco with an image of the local Target superimposed. Note that the store in San Francisco does not have the name below the logo. Furthermore, the logo sits on top of an AMC sign and both Target and AMC use the same red color. This makes the two signs seem like one.

Because Target is very familiar to the participants (there is a Target store near the university), they must have expected a sign and logo similar to the local store. Figure 7.10 shows the differences in the two logos. Participants who did not see this difference in the encoding stage (No Preview and Map View), were possibly expecting a similarity between the local Target sign and the one in San Francisco. Participants in the Street View saw the difference at the encoding stage and thus had a better chance at recognizing the store.

Target. To validate this plausible explanation, I conducted a chi-square for those participants who rated Target at the highest familiarity (33 participants at level 5) comparing location 


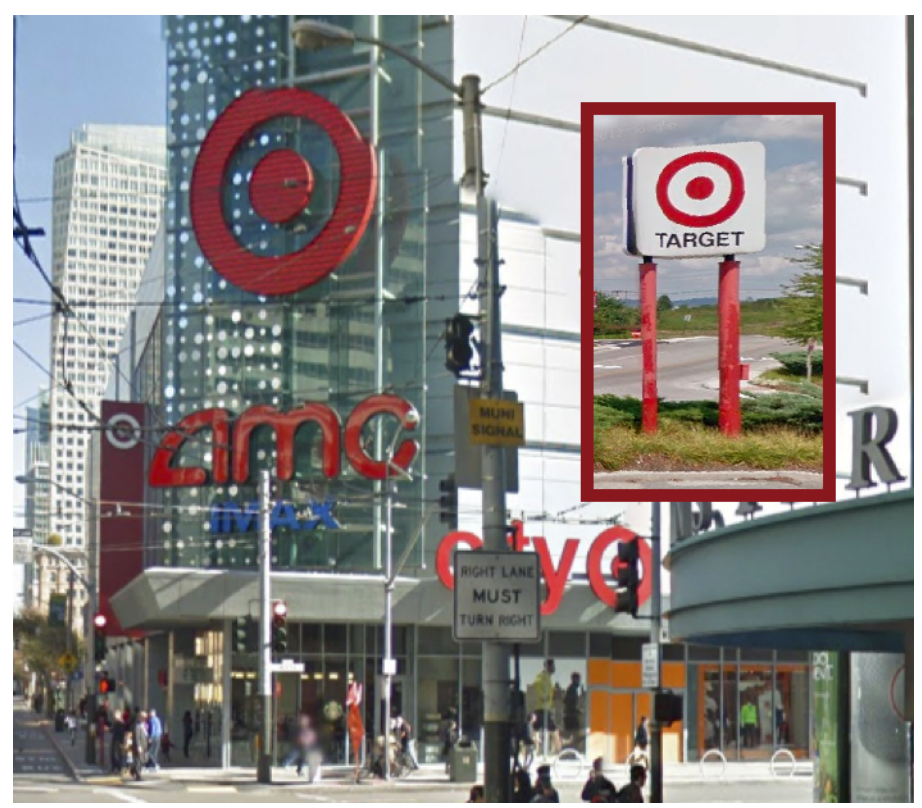

Figure 7.10: Two views for Target. The main image is the view for the Target location in San Francisco. The embedded image in the top right of the figure is the local image for Target

recognition across the three conditions. The chi-square shows a significant difference (Pearson chi-square $p=.006)$. On a subsequent pair-wise analysis, Street View vs. No Preview was significant $(p=.001)$ a well as Street View vs. Map View $(p=.018)$. Figure 7.11 shows the counts for these results. No participant from the Street View missed the Target location. The Map View, on the other hand, had more participants missing Target than identifying it. I also ran this analysis for the other levels of familiarity $(4,3,2,1)$ and found no significant differences.

Peets Coffee $\&$ Tea. I conducted a chi-square for those participants who rated Peets Coffee \& Tea at the lowest familiarity level (40 participants at level 1) comparing location recognition and the three conditions. The chi-square shows a significant difference (Pearson chi-square $p=.003)$. On a subsequent pair-wise analysis, Street View vs. No Preview was significant $(p=.001)$ as well as Street View vs. Map View $(p=.016)$. Figure 7.12 shows the counts for these results. I also ran this analysis for the other levels of familiarity $(2,3,4,5)$ and 


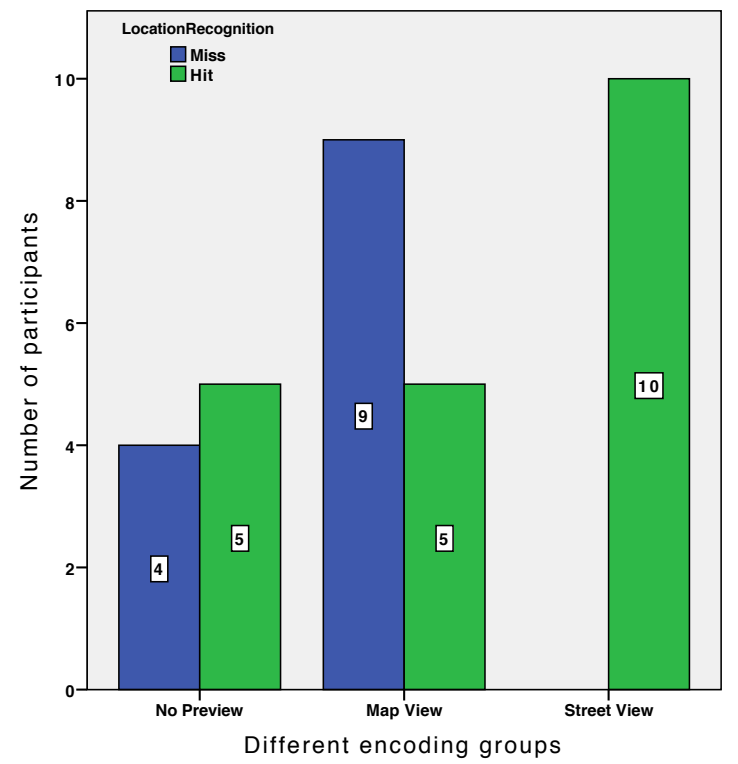

Figure 7.11: Recognition (hit or miss) for Target Location among the participants with highest recognition for this business

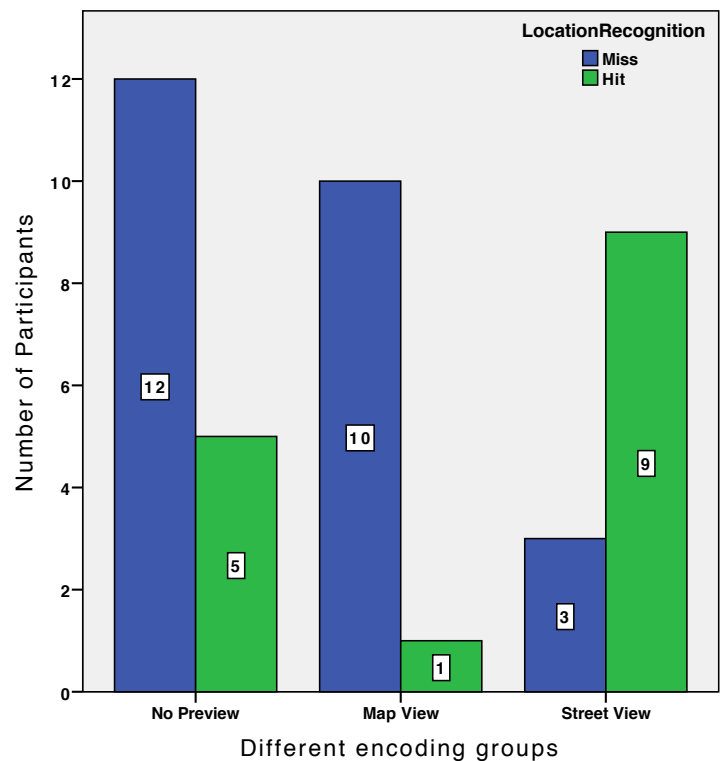

Figure 7.12: Recognition (hit or miss) for Peets Coffee \& Tea location among the participants with lowest recognition for this business 
found no significant differences.

$A A U$ I conducted a chi-square for those participants who rated AAU at the lowest familiarity level (51 participants at level 1) comparing location recognition and the three conditions. I found no significant difference (Pearson chi-square $p=.077$ ) for this test. There was no significant difference for AAU at any familiarity level.

\subsubsection{Task Frequency and Task Recall}

Figure 7.13 shows the average frequency level of each task rated by the participants. "Do grocery shopping" was rated as the most frequently daily to-do tasks, while "open a credit card" was rated as the most infrequently daily to-do task. This result is consistent with the participants involved in the study; all of them are students living a college life, and most of them are undergraduates who have never opened a credit card before. I took a further look at this particular task to see whether this factor influenced the task recall performance differently among three groups. I conducted a chi-square for those participants who had never opened a credit card before (20 participants), comparing task recall performance and the three conditions. The result shows a significant difference (Pearson chi-square $p=.006$ ) among the three groups. For further pair-wise analysis, No Preview vs. Map View was significant $(p=.001)$ as well as No Preview vs. Street View $(p=.024)$ was significant. But no significance was found between Map view and Street View.

\subsubsection{Gender and Memory}

Gender. Given the higher number of female participants (43 female vs 17 males), I wanted to confirm that gender did not play a role in the results. I conducted three two-factor ANOVAs to explore an effect of gender and the three measures of performance: complete 


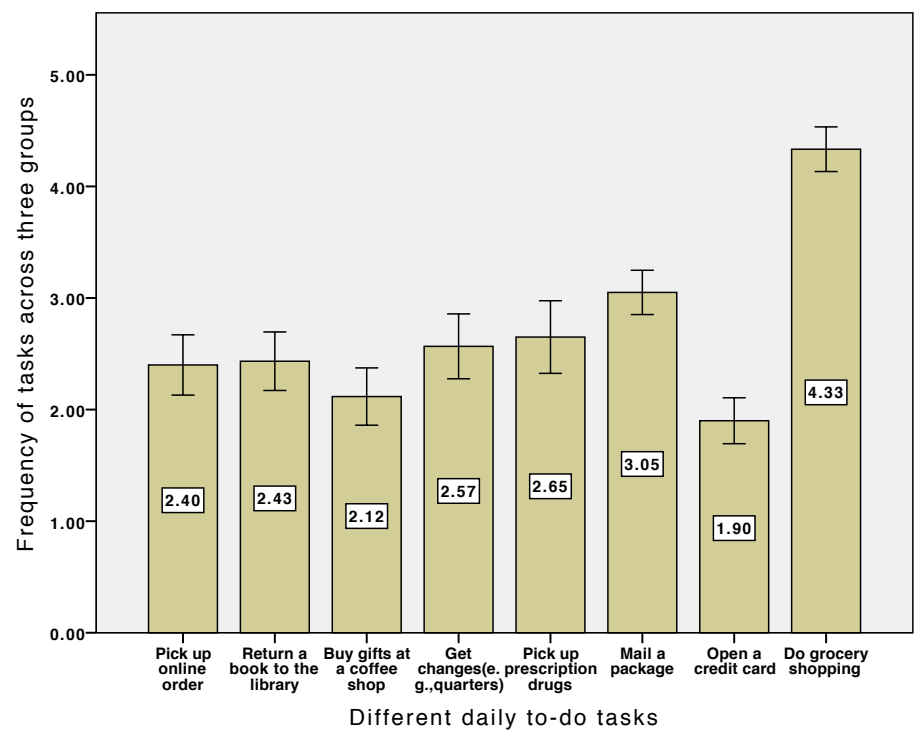

Figure 7.13: Frequency level with each task used in the study

recall, location recognition and task recall. There was no interaction effect in any of the three analysis between gender and the experimental conditions for each of the three measures of performance. Gender, as a main factor, also had no significant effect on complete recall performance $(F(2,54)=.267, p>.05)$, location recognition $(F(2,54)=.935, p>.05)$, nor task recall performance $(F(2,54)=.121, p>.05)$.

Memory Ability. I also conducted three two-factor ANOVAs to explore an effect of memory ability and the three measures of performance: complete recall, location recognition and task recall. There was no interaction effect in any of the three analysis between memory and the experimental conditions for each of the three measures of performance. Memory, as a main factor, also had no significant effect on complete recall performance $(F(11,32)=1.037, p=$ $0.439)$, location recognition $(F(11,32)=1.772, p=0.102)$, nor task recall performance $(F(11,32)=1.484, p=0.186)$. 


\subsubsection{Familiarity Level}

I was concerned that participants' different familiarity levels with the experimental city (San Francisco), might have had an impact on the validity of the data. Therefore, I subsequently compared the results of recall performance using an independent t-test between participants who were and were not familiar with the experimental city. I found no significant difference in Complete Recall ( $\mathrm{p}=.22)$, Location Recognition $(\mathrm{p}=.75)$, nor Task Recall ( $\mathrm{p}=.32)$ between the participants who were familiar with the experimental city and those who were not.

\subsubsection{Familiarity with Daily Errands}

Interestingly, I found that people mixed up their daily errands with some of the study tasks. Sometimes participants remembered the correct task but with the wrong location. Seven participants in the No Preview group mixed up the study locations with real locations: three of them remembered to pick up medicine from CVS rather than Walgreens, as CVS is the normal place they would go in their daily life; one participant remembered to stop by Opticians to pick up the medicine; and three participants remembered to stop by other banks such as Beal Bank, PNC Bank and Chase Bank rather than Citibank.

In addition, ten participants in the Map View group remembered the wrong locations: five of them thought they might need to go to Starbucks (the encoded location is Peets Coffee \& Tea); one participant thought he needed to pick up medicine in CVS; two participants thought it was USPS rather than FedEx, and two participants thought they remembered another bank to open a credit card (Union Bank and Chase).

However, only three participants in the Street View group recognized the wrong location: one was not sure whether it was Walgreens or CVS to pick up the medicine; one participant considered it was Union Bank rather than Citibank since both logos are blue, and one 
participant thought it was Starbucks rather than Peets Coffee \& Tea.

It seems that Street View provided more contextual information for participants to encode and match at the retrieval stage to support a more successful retrieval. For example, participants in the Street View commented, "I think I have seen this white and black sign before, and it also starts with P-something," or "I remember seeing this Kaplan, and I remember seeing this (AAU sign), and I remember what was across the street."

\subsection{Design Implications}

Revisiting my research questions I found that using Street View during the encoding stage of a prospective task in an LBR had a significant effect in the retrieval stage three days later. Participants in the Street View group had better performance in all three measures: Location Recognition, Task Recall and Complete Recall. I posit that the Street View at the encoding provides a more compatible view to the retrieval stage than the other two conditions. Encoding using Street View includes the logo of the desired location, the building, and the surrounding landmarks, thus providing more information that could match the view people encounter at the retrieval stage. This finding matches work done by Einstein

and McDaniel [Einstein et al., 1996]: prospective remembering is sensitive to the match of features between encoding and retrieval stage. Designing LBRs encoding stage using Street View is more in line with how human prospective memory works.

I found that lack of familiarity (e.g., AAU, Peets Coffee \& Tea) could hinder performance in the retrieval stage but that, once again, Street View could in fact close the gap and still provide strong support for those locations with which participants had low (or no) recognition. The encoding match with the cue removed the deficit of not knowing the business logo. This result demonstrated that people who were familiar with the target 
location were influenced in how they recognized the location. It also indicated that different encoding interfaces might be needed between when people are in a new place and when they are in a familiar place. Future LBR designers should address the factor of familiarity when presenting location information.

Furthermore, when the distinctiveness and the associativity [McDaniel and Einstein, 2000] of a location is not what is expected, participants in the Map View and the No Preview groups were at a disadvantage, as shown in the Target example.

Street View is particularly useful when people need to complete a task at a specific location. In previous work, Wang and Pérez-Quiñones [Wang and Perez-Quinones, 2015] classified the location-based to-do tasks based on different location types: sometimes a task has to be done at a specific location and sometimes a task can be completed at chains or at any one of similar stores. For example, a task "to get allergy medicine" can be completed at any Walgreens rather than a specific one, or at other pharmacies or drug stores. As seen in the study, participants remembered to get allergy medicine, but they remembered the CVS rather than Walgreens, since the task could be completed at either location. For the goal of completing the task to get allergy medicine, CVS and Walgreens are equal candidates. Future LBRs should provide support to encode location information based on the types of tasks rather than the specific location. Furthermore, the retrieval stage must then consider alternate locations where the task can be completed, as in the CVS and Walgreens example above.

The findings focus on the importance of using prospective memory theory in the design of future LBR systems. The importance of the cue [Vortac et al., 1995] for retrieval was confirmed by the findings. Participants who saw a street view of the desired location were able to recognize more locations and recall more tasks. The match between encoding and retrieval [Einstein and McDaniel, 1990] was essential for better performance. 


\subsection{Summary}

Can I improve the design of LBRs without improving the location-sensing technology? Can I improve them without an overly complicated task setup [Cakmak and Basoglu, 2012]? Based on the findings, the answer is yes on both counts. This study shows that just by showing a street view in the encoding stage, instead of the typical map view, performance of location recognition and task recall is improved. Note that in my experiment, I never relied on an alert, participants simply relied on their memory. My work provides evidence as to how I can depend on prospective memory to improve the design of an LBR system while potentially reducing the over-reliance on external memory aids.

It is worth noting that although a user could remember to do something when they see a location, deciding to do the task is more complex than just being at the desired location. Doing the task "remember to buy milk," for example, depends not only on location but also on being able to do the task at the particular time when I approach the location. It makes more sense to buy the milk as I pass the supermarket on the way home rather than on the way to work. My work, as reported here, focuses only on the triggering of the alert based solely on location detection and not on the appropriateness of doing the task at that particular time.

This study has implications for the design of current LBRs. Current systems should provide more support in matching the encoding stage to the eventual cue in retrieval stage. A simple way to accomplish this is to make use of existing Street View in commercial mapping software instead of just Map View to increase the match between encoding and retrieval stages. This should make LBRs more compatible with how prospective memory works. 


\section{Chapter 8}

\section{The Impact of Familiarity}

This chapter describes my second empirical user study of how the familiarity with the locations impacts the recall performance on location recognition and task recall. The study had two experimental sessions. The study's setup and procedure followed the first one. This study was approved by the Virginia Tech Institutional Review Board with approval \# 15-676 on October 23, 2015.

\subsection{Experimental Design}

In this study, I am interested in how familiarity with cities and places influence people's abilities to remember location-based to-do tasks. For city, I used a home city considered as a familiar location context and a traveling city as an unfamiliar location context. The participants all lived and/or worked in the home city and most had never visited the traveling city. For place, I used business locations typically used in location-based to-do tasks. For example, a drugstore is used to pick up a prescription. I measured familiarity as a userprovided familiarity rating with the business and the business logo. Therefore, participants 


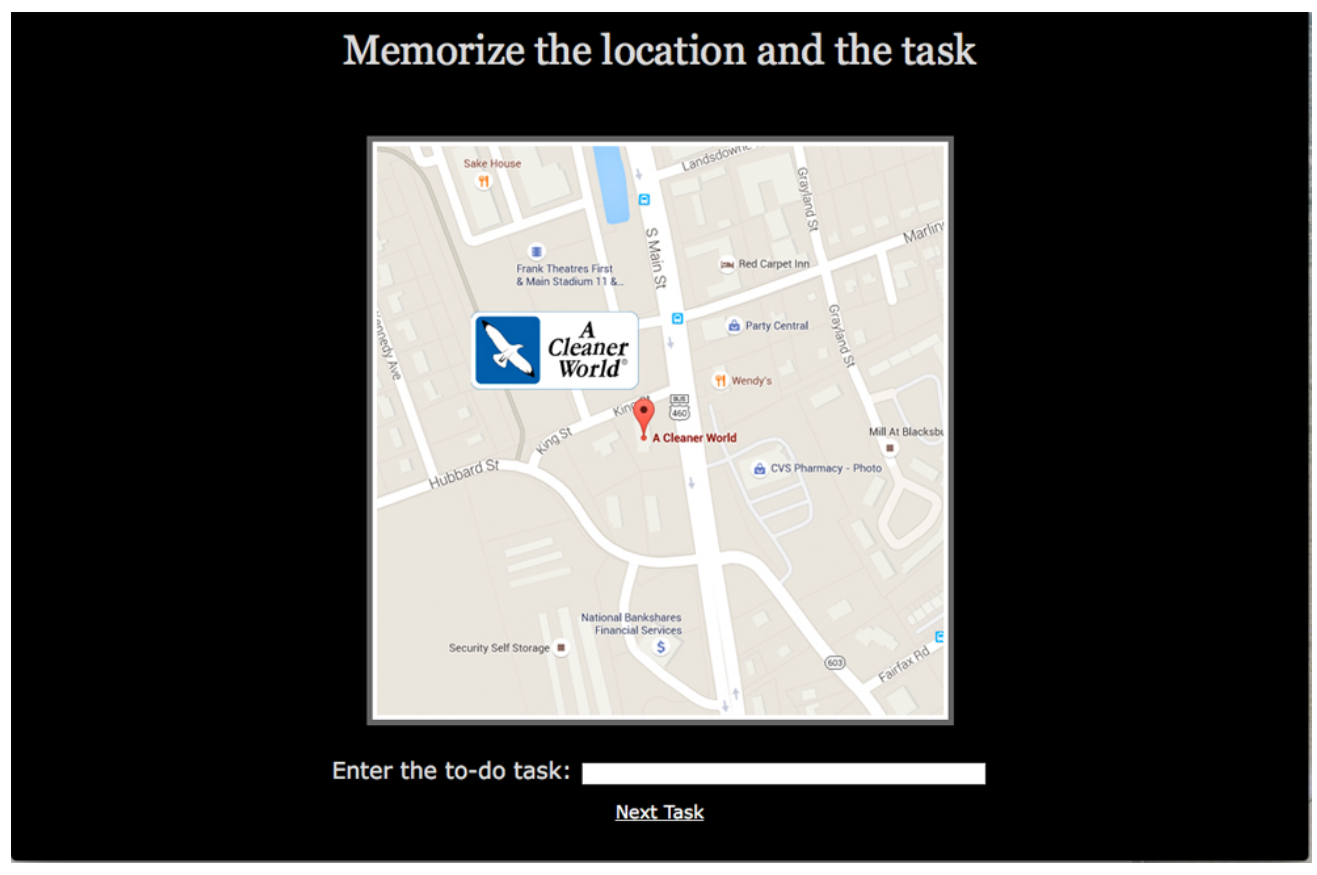

Figure 8.1: Map+Logo View for entering the to-do task, showing the location of A Cleaner World.

would be familiar with the logo for Target, and it would be easy to identify even if they had never visited a Target store in the traveling city. Eight places were selected from the home city and another eight similar places were selected from the traveling city. These were presented to the participants using three different encoding methods based on the experimental group (more below).

This study consisted of two sessions, each session took approximately 30 minutes. Both sessions were conducted in the same usability lab on campus. Participants were asked to memorize eight predefined location-based to-do tasks during the first session. Three days later, they were asked to come back to recognize the locations and recall the associated to-do tasks while watching a video.

The experiment was designed as a between-subjects study with six different groups: two types of cities (home, traveling) and three different encoding methods (Map+Logo View, Street 


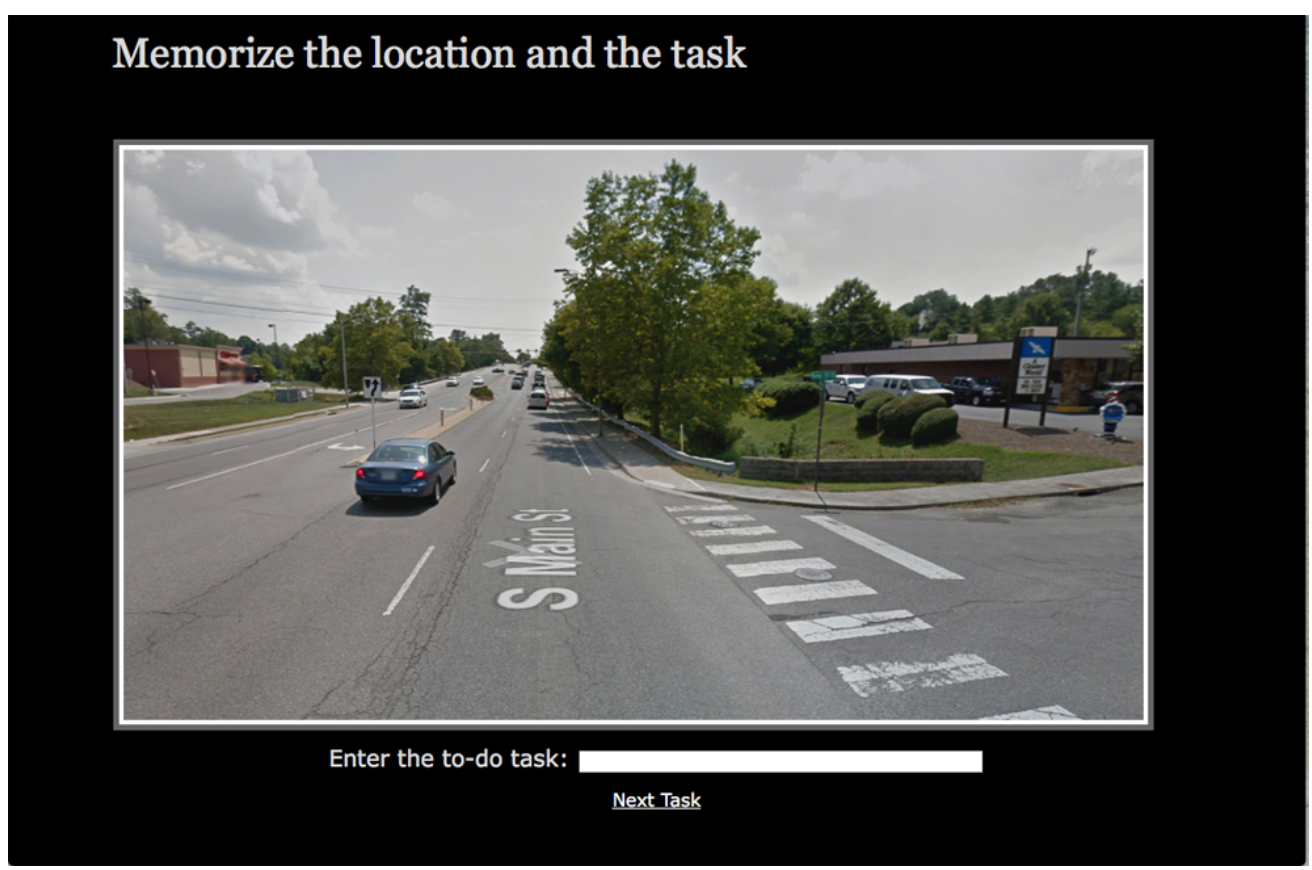

Figure 8.2: Street View for entering the to-do task, showing the location of A Cleaner World.

View and Interactive Street View). The independent variables were the city and the encoding methods. The dependent variables were three measures of performance in accomplishing the prospective to-do tasks and the time spent encoding the tasks as a measure of effort.

Map+Logo View Group Participants in this group saw the designated place on a Google Map, with the business logo (perceptual icon [Thorndyke et al., 1980]) on it, as shown in Figure 8.1. This view can be characterized as supporting survey knowledge from an allocentric perspective [Thorndyke and Goldin, 1983].

Street View Group Participants in this group saw an image of the designated place captured from Google Street View (see Figure 8.2 for an example). Participants experienced this location information from an egocentric view that would match the navigation experiences at the recall stage. This view allowed participants to expand their landmark knowledge [Thorndyke and Goldin, 1983] as it allowed them to build up information about buildings around the target location but with a fixed, experimenter-controlled view. 


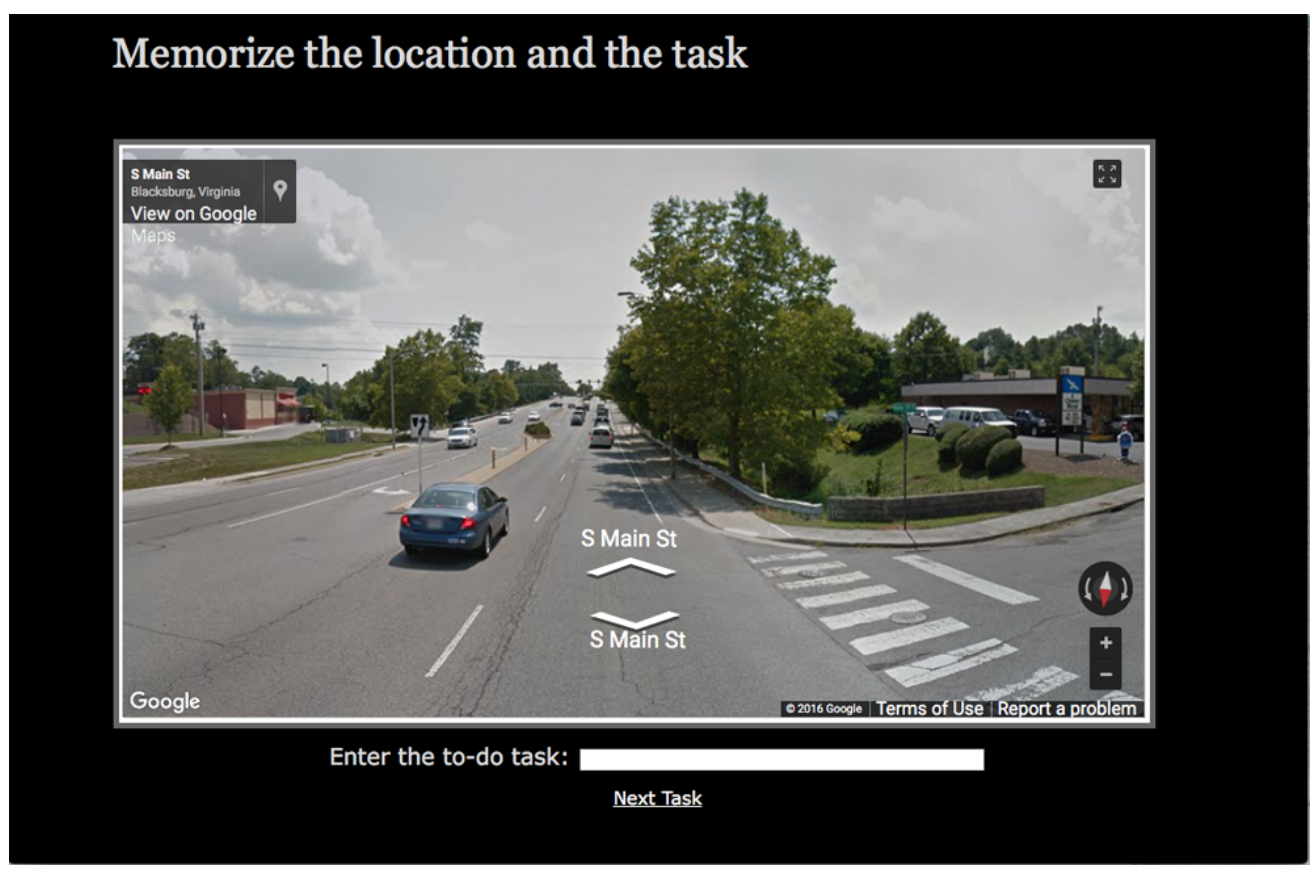

Figure 8.3: Interactive Street View for entering the to-do task, showing the location of A Cleaner World.

Interactive Street View Group Participants in this group saw an Interactive Google Street View (see Figure 8.3) of the designated place, which enabled participants to interact with the interface. The interaction allowed participants to see not only the desired place but a full 360 degrees of the surrounding area. They could move their perspective around to get a better sense of the neighborhood of the designated place. I expected that the Interactive Street View would enhance participants' landmark knowledge and develop additional procedural knowledge [Thorndyke and Goldin, 1983]. 


\subsection{Study Setup}

\subsubsection{Participants}

I recruited sixty students using the Experiment Management System used by Psychology Department. The students participated for course credit. The participants were randomly assigned into each of the two groups: home (familiar) and traveling (unfamiliar) city. Within each city group, the participants were further randomly subdivided into one of the three interfaces (ten participants per condition), each group seeing a different encoding interface. Participants ranged in age from 18 to 32 years old, with an average age of 20 years old. There were 20 males, 39 females and one participant who did not disclose the gender. Participants were students in a variety of majors from STEM, management, psychology, humanities, and medical fields.

The home city used in the study is Blacksburg where the university is located. The 30 participants in the home city rated the familiarity of the city as 4.27 out of 5 , where 1 means very unfamiliar and 5 means very familiar. Participants had lived in the city for a minimum of 6 months and a maximum of 7 years at the time of the experiment. The traveling city used was San Francisco, which is located just over 2,000 miles from the home city. The 30 participants in the traveling city rated the familiarity of the city as 1.30 out of 5 . At the time of the experiment, 22 out of 30 participants had never visited San Francisco before; the other 8 participants had traveled there no more than twice.

Participants' background information is listed in Table 8.1 for each group. 


\begin{tabular}{|c|c|c|c|c|}
\hline & & Map+Logo View & Street View & $\begin{array}{l}\text { Interactive Street } \\
\text { View }\end{array}$ \\
\hline Familiar City & $\begin{array}{l}\text { Num. } \\
\text { Avg. Age } \\
\text { Gender } \\
\text { Program of } \\
\text { study }\end{array}$ & $\begin{array}{l}10 \\
21.8(19-32) \\
6 \text { females and } 4 \text { males } \\
7 \text { bachelor, } 1 \text { master } \\
\text { and } 2 \text { doctorate }\end{array}$ & $\begin{array}{l}10 \\
19.3(18-23) \\
6 \text { females and } 4 \text { males } \\
10 \text { bachelor }\end{array}$ & $\begin{array}{l}10 \\
20.1(18-25) \\
6 \text { females, } 3 \text { males and } \\
1 \text { undisclosed } \\
8 \text { bachelor, } 2 \text { masters }\end{array}$ \\
\hline Unfamiliar City & $\begin{array}{l}\text { Num. } \\
\text { Avg. Age } \\
\text { Gender } \\
\text { Program of } \\
\text { study }\end{array}$ & $\begin{array}{l}10 \\
19.5(18-22) \\
7 \text { females and } 3 \text { males } \\
10 \text { bachelor }\end{array}$ & $\begin{array}{l}10 \\
21.1(18-28) \\
7 \text { females and } 3 \text { males } \\
8 \text { bachelor, } 2 \text { master }\end{array}$ & $\begin{array}{l}10 \\
18.5(18-20) \\
7 \text { females and } 3 \text { males } \\
9 \text { bachelor, } 1 \text { master }\end{array}$ \\
\hline
\end{tabular}

Table 8.1: Participants' background information

\subsubsection{Procedure}

Prior to their arrival, each participant was randomly assigned into one of the six encoding groups. Upon arrival to the first session, each participant filled out a consent form and completed a background questionnaire. To start the first session, I presented eight to-do tasks to be done at particular places. I asked them to enter the task information via the assigned interface. I informed the participants that they could type any information into the interface that would help them remember the task three days later. There was no time limit for participants to memorize these tasks. All participants in each city received the exact same instructions and to-do tasks. The first session ended with the completion of memorizing the eight tasks. The eight task scenarios in the traveling city were borrowed from the first empirical study. The predefined eight-task scenarios to perform in the home city are listed below:

1. The fall season is coming and you suffer from seasonal allergies. You would like to stop by Target to pick up your allergy prescription drugs on your way home. 
2. You plan to go fishing this weekend with several friends. Blacksburg Feed \& Seed Co is the place offering professional fishing supplies. You pass by this store everyday when you are driving home, so you decide to stop by this store to get some fishing supplies.

3. You dropped your suits off at the dry cleaners, A Cleaner World, a few days ago. Your laundry is ready to pick up.

4. You live in an apartment, and you need some quarters to use in the washer and dryer for laundry. There is a Wells Fargo bank on your way home, so you want to get some quarters at this bank.

5. Your mom's birthday is coming up. Kent Jewelry is offering limited specials. You would like to buy your mom a piece of jewelry for her birthday present.

6. You ordered a wireless printer online, but shipping is expensive. You decided to pick it up at the Best Buy store and save the shipping cost. Pick up the printer as you drive by Best Buy.

7. You would like to mail a package to one of your friends in Canada. Stop by the US Postal Office to drop the package.

8. You received a promotional letter from Carter Bank and Trust for a new credit card. You need to stop by the bank to sign some papers. You decide to do so the next time you drive by the bank.

Participants were asked to come back three days later to complete the second session. In this session, I asked participants to watch a video taken from the viewpoint of a car's front windshield, simulating driving around the city. A separate video was shown for each of the two cities. Participants in the home city watched a video lasting 12 minutes and 19 seconds, while participants in the traveling city group watched a slightly shorter video (10 minutes 
and 49 seconds). Both videos showed a car driving through the city and passing by the relevant places for all the tasks. Participants need to pause the video once they recognized a place for one of their tasks and to report the associated to-do task. While watching the video, participants were also required to count the traffic lights to simulate the attention that would be required if they were navigating the vehicle. This was intended to simulate a prospective task being embedded within another task.

At the end of the video, I asked the participants to report any additional task that they remembered but missed the location during the video. I then asked participants to fill out a post-test questionnaire. As a wrap-up, I conducted a short interview to explore participants' perceptions of the interface used.

\subsubsection{Data Collection}

For both sessions, I screen recorded the interactions between participants and the interface, and audio recorded participants' comments. In the first session, I recorded the time participants spent on memorizing the tasks, measured from the time participants first opened the encoding interface, until the participants clicked the "I am done" button to close the interface. I also collected the encoding messages participants typed into the computer for each task. In the background questionnaires, I asked participants to report their demographic information. In the second session, I collected participants' performance under three measures. Location Recognition was defined as the number of times participants successfully recognized the place from the video. Task Recall referred to the number of times participants successfully remembered the task either when they recognized the location during the experiment or at the end of the session. When participants correctly recalled the task at the correct location, I counted this as a Complete Recall. In the post-test questionnaire, I 
collected the ratings of the familiarity levels with the city and the place of each of the tasks encountered in the experiment. Participants rated each city and place on a scale from 1 to 5, where 1 means very unfamiliar and 5 means very familiar.

\subsection{Results}

\subsubsection{Familiar City (home)}

Hypothesis 1: When people are familiar with a city, performance (as measured in this study) is not affected by different interface styles because the interfaces do not provide additional support to an already developed spatial knowledge.

I conducted three one-way between-subjects ANOVA analyses to compare the effect of the encoding method on Complete Recall, on Location Recognition, and on Task Recall under the three interfaces (Map+Logo, Street, and Interactive Street View) for participants in their home (familiar) city. There was no significant effect of encoding method at the $p<.05$ level for the three interfaces under Complete Recall $[F(2,27)=.416, p=.664]$, nor under Location Recognition $[F(2,27)=.794, p=.462]$ nor under Task Recall $[F(2,27)=.310, p=.736]$.

Taken together, these results suggest that the interface style did not have an effect on any of the three performance measures in the home city. Specifically, our results suggest that when users are familiar with the city, the different encoding interfaces used for capturing a prospective task have little influence in the recognition of place and the recall of the to-do task at a later time. Presumably, the accumulated familiarity of the city includes enough survey, landmark, and procedural knowledge leading to a rich mental map of the city and thus reducing the cognitive aid afforded by some of the interfaces. 


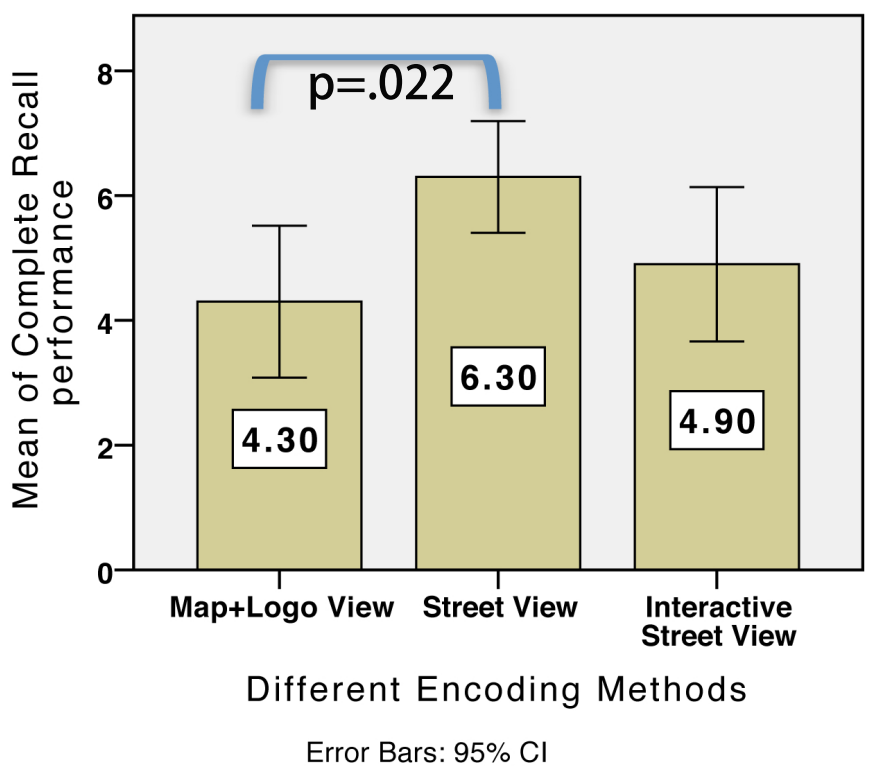

Figure 8.4: Complete Recall performance in an unfamiliar city for all three encoding methods, $\mathrm{F}(2,27)=4.238, \mathrm{p}=.025$. Based on Tukey HSD, Street View had significantly better complete recall than the Map+Logo View.

\subsubsection{Unfamiliar City (traveling)}

Hypothesis 2: When people are unfamiliar with a city, performance (as measured in this study) using the Interactive Street View improves because the interface provides additional contextual information (landmark and procedural knowledge).

I conducted a one-way between-subjects ANOVA to compare the effect of encoding method on Complete Recall at the $p<.05$ level between the three interfaces (Map+Logo, Street, and Interactive Street View) for participants in the traveling (unfamiliar) city (see Figure 8.4). There was a significant effect of encoding method on Complete Recall for the three interfaces $[F(2,27)=4.238, p=.025]$. Post hoc comparisons using the Tukey HSD test indicated that the mean score for the Street View interface $(\mathrm{M}=6.30, \mathrm{SD}=1.252)$ had a significantly better performance than the Map+Logo View $(\mathrm{M}=4.30, \mathrm{SD}=1.703), \mathrm{p}=.022$. However, the performance in the Interactive Street View $(\mathrm{M}=4.90, \mathrm{SD}=1.729)$ did not significantly differ 


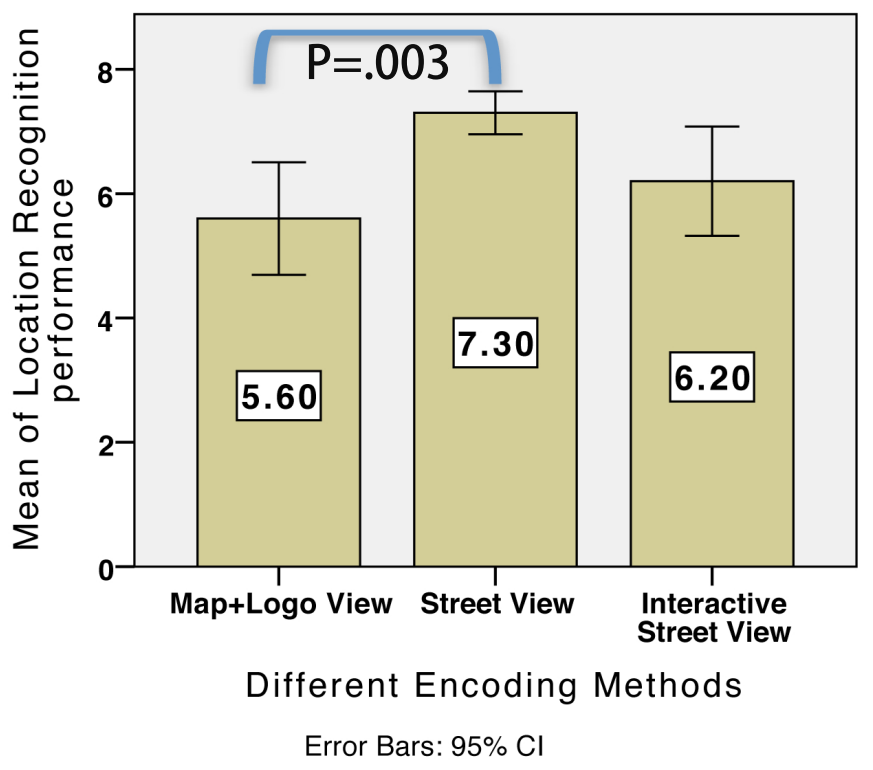

Figure 8.5: Location Recognition performance in an unfamiliar city for all three encoding methods, $\mathrm{F}(2,27)=6.668$, $\mathrm{p}=.004$. Based on Tukey HSD, Street View had significantly better Location Recognition performance than Map+Logo View.

from performance in the Map+Logo View or Street View.

I conducted another one-way between-subjects ANOVA to compare the effect of the encoding method on Location Recognition between the three interfaces. There was a significant effect of encoding method on Location Recognition at the $p<.05$ level for the three interfaces $[F(2,27)=6.668, p=.004]$ (see Figure 8.5). Post hoc comparisons using the Tukey HSD test indicated that the mean score for Street View $(\mathrm{M}=7.30, \mathrm{SD}=.483)$ was significantly higher than the Map+Logo View $(\mathrm{M}=5.60, \mathrm{SD}=1.265), \mathrm{p}=.003$. However, the Interactive Street View condition $(\mathrm{M}=6.20, \mathrm{SD}=1.229)$ did not significantly differ from the Street or Map+Logo View.

I conducted a third one-way between-subjects ANOVA to compare the effect of the encoding method on Task Recall between the three interfaces. There was no significant effect of encoding method on Task Recall at the $p<.05$ level for the three interfaces $[F(2,27)=$ 
$1.984, p=.157]$

I found no evidence to support Hypothesis 2. Participants using the Interactive Street View did not have better performance than the other groups. Street View, however, had a significantly better Complete Recall and Location Recognition performance than Map+Logo View. Surprisingly, the added interaction of the Interactive View did not benefit performance. Later, I provide a plausible explanation for this finding based on the comments provided by the participants.

\subsubsection{Familiarity of Places in a Familiar City}

All participants in the home (familiar) city rated each of the places (eight locations) for familiarity using a 1-5 scale. The average familiarity ratings for each place is shown in Figure 8.6. The figure shows that four places (Target, Wells Fargo, Best Buy, USPS) were rated with an average rating above 4 (familiar). The other four places (Town Feed \& Seed Co, A Cleaner World, Kent Jewelry, Carter Bank and Trust) had an average rating under 3 (neutral). Using this division of familiar and unfamiliar places within a familiar city, I further analyzed differences between the three interface conditions.

\section{Hypothesis 3: Familiar Places in a Familiar City.}

Hypothesis 3: When people are familiar with a place in a familiar city, performance (as measured in this study) is not affected by different interface styles because city and place familiarity are proxies for the spatial knowledge required for this task.

The four familiar places were rated at an average at 4.31 (out of 5). Using only these four familiar places in the familiar city, I analyzed performance for these tasks. I performed a chi-square test of independence to examine the relationship between the encoding methods 


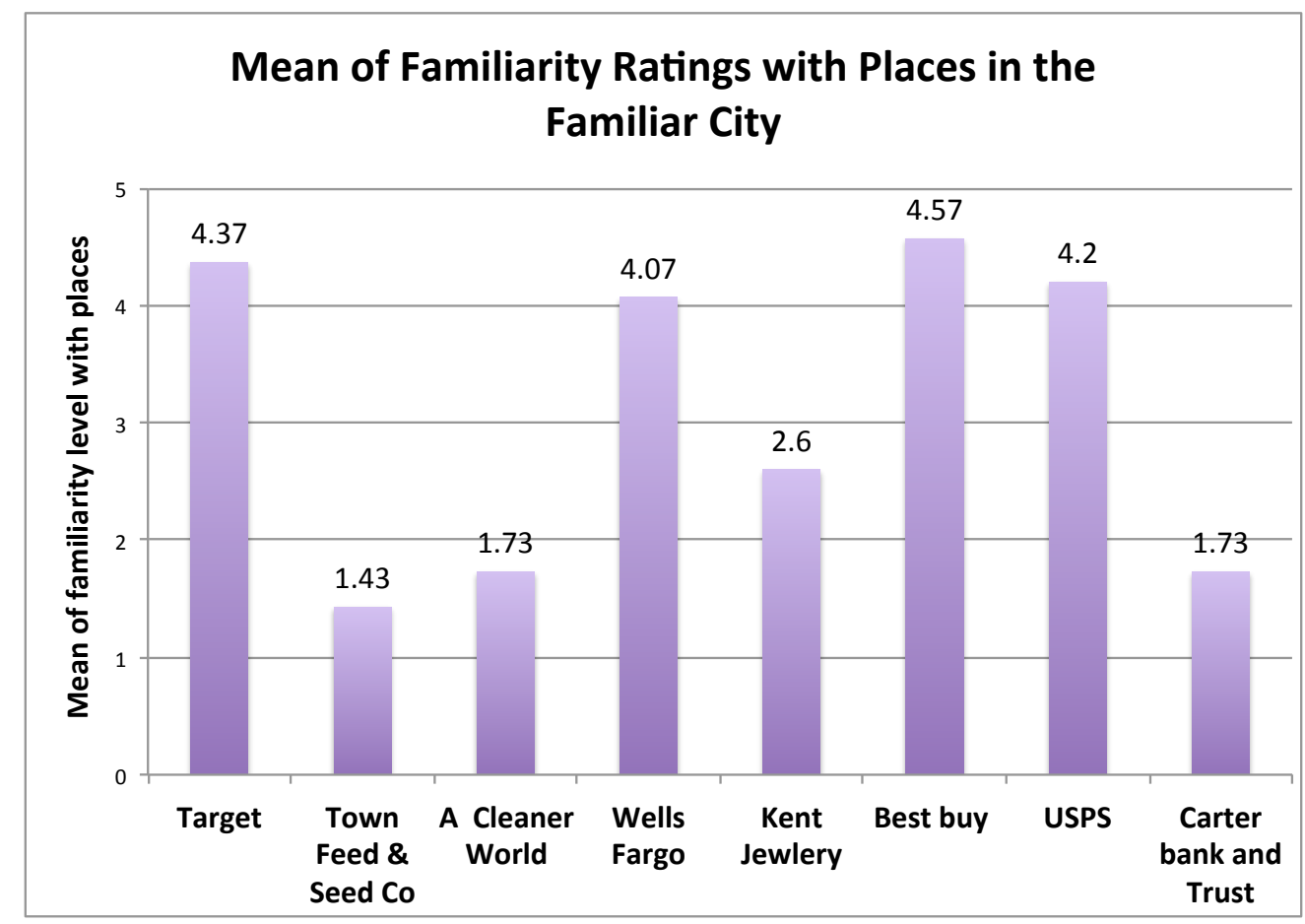

Figure 8.6: Average familiarity ratings with places in familiar city

and performance for this subset of the data. Neither the Complete Recall $\chi 2(2, N=120)=$ $1.867, p=.393$ nor the Task Recall was significant $\chi 2(2, N=120)=2.274, p=.321$.

For Location Recognition, I was not able to use a chi-square test, because the data set did not meet the assumptions (some of the cells had an expected count of less than five). Instead, I conducted a Fisher's Exact Test between all pairs of three encoding methods on Location Recognition. There was no significant difference among the groups. All three performance measures under three interfaces are shown in Figure 8.7.

\section{Increasing Familiarity of Places in a Familiar City}

A logistic regression was performed to ascertain the effect of familiarity with places (on a scale 1..5) on the likelihood that participants will successfully recognize that place and recall the associated to-do task in the familiar city. Under the measure of Complete Recall, the logistic 


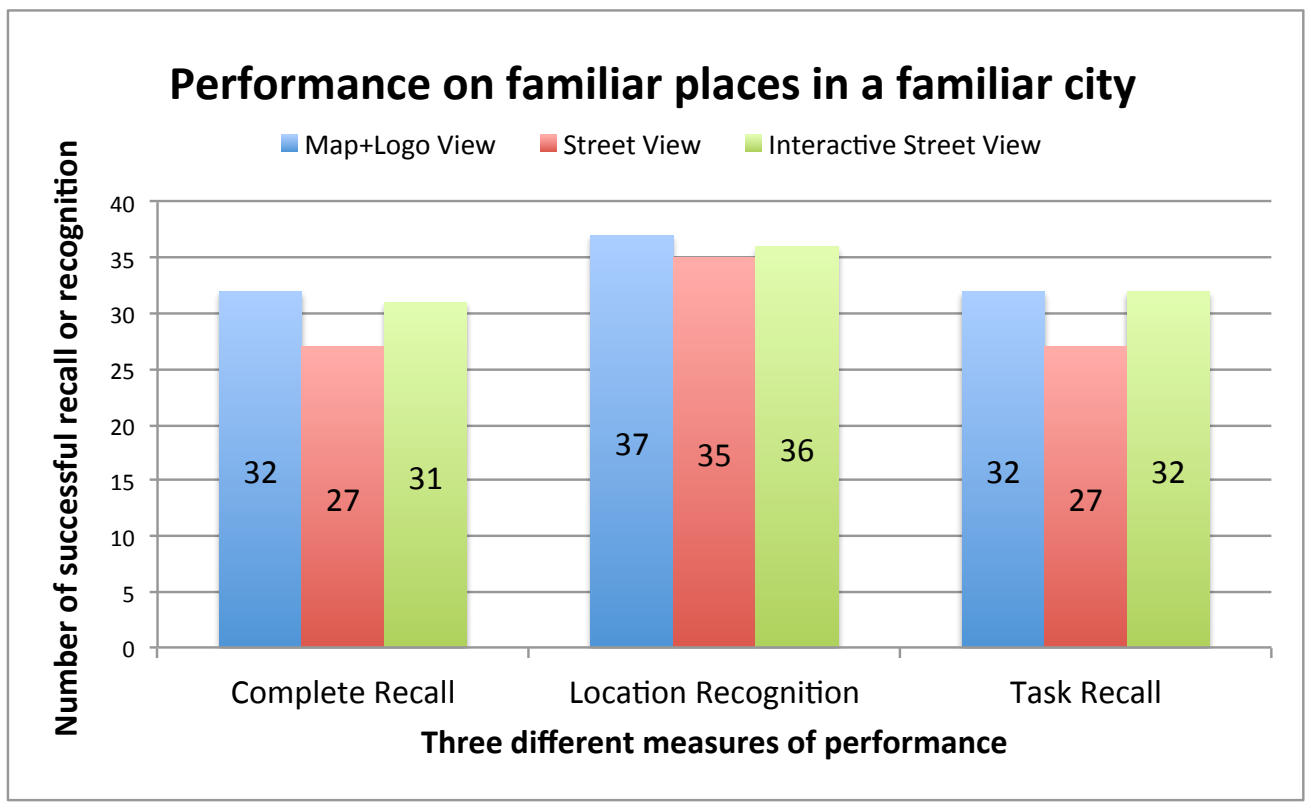

Figure 8.7: Performance of familiar places in a familiar city for all three encoding methods.

regression model was statistically significant, $\chi 2(1)=23.630, p=.000$. The familiarity with places had a significant effect on the recall performance: increasing familiarity with places was associated with an increased likelihood of a better Complete Recall performance.

Under the measure of Location Recognition, the logistic regression model was also statistically significant: $\chi 2(1)=57.292, p=.000$. The familiarity with places had a significant effect on the recognition performance, increasing familiarity with places was associated with an increased likelihood of a better Location Recognition performance. However, the logistic regression model didn't show any significant relationship between the familiarity with places and Task Recall, $\chi 2(1)=1.420, p=.233$.

The results show support for Hypothesis 3. As with Hypothesis 1, familiarity with the city implies that a rich mental model might already be in place and thus any added support from the three different interfaces did not have any effect on performance. Furthermore, the logistic regression shows that increasing familiarity of locations increases the likelihood of 
recognizing the locations for the tasks to be performed in this study.

\section{Hypothesis 4: Unfamiliar Places in a Familiar City.}

Hypothesis 4: When people are familiar with the city, but not familiar with the place, performance (as measured in this study) improves with Interactive Street View because the interface provides additional contextual information (landmark and procedural knowledge).

The four unfamiliar places were rated at an average at 1.87 (out of 5). Using only the four unfamiliar places in the familiar city, I analyzed performance for these tasks. I performed three chi-square tests of independence to examine the relationship between the encoding methods and performance under different measures. The chi-square tests revealed that there was no significant difference among the three encoding methods under Complete Recall $\chi 2(2, N=120)=3.360, p=.186$, nor under Location Recognition $\chi 2(2, N=120)=$ $4.964, p=.084$, nor under Task Recall $\chi 2(2, N=120)=2.455, p=.293$. Performance under the three measures is shown in Figure 8.8.

According to the results, I found no evidence to support Hypothesis 4. I assume that because of the familiarity with the city, participants had created a good mental map with visual information of the most surrounding places: even the minimum information (Map+Logo View) I provided is enough to learn the particular location effectively although they are not familiar with them. In the next section, I discuss the comments from the participants. These comments might provide a plausible explanation as to why this interface did not make a difference in performance. 


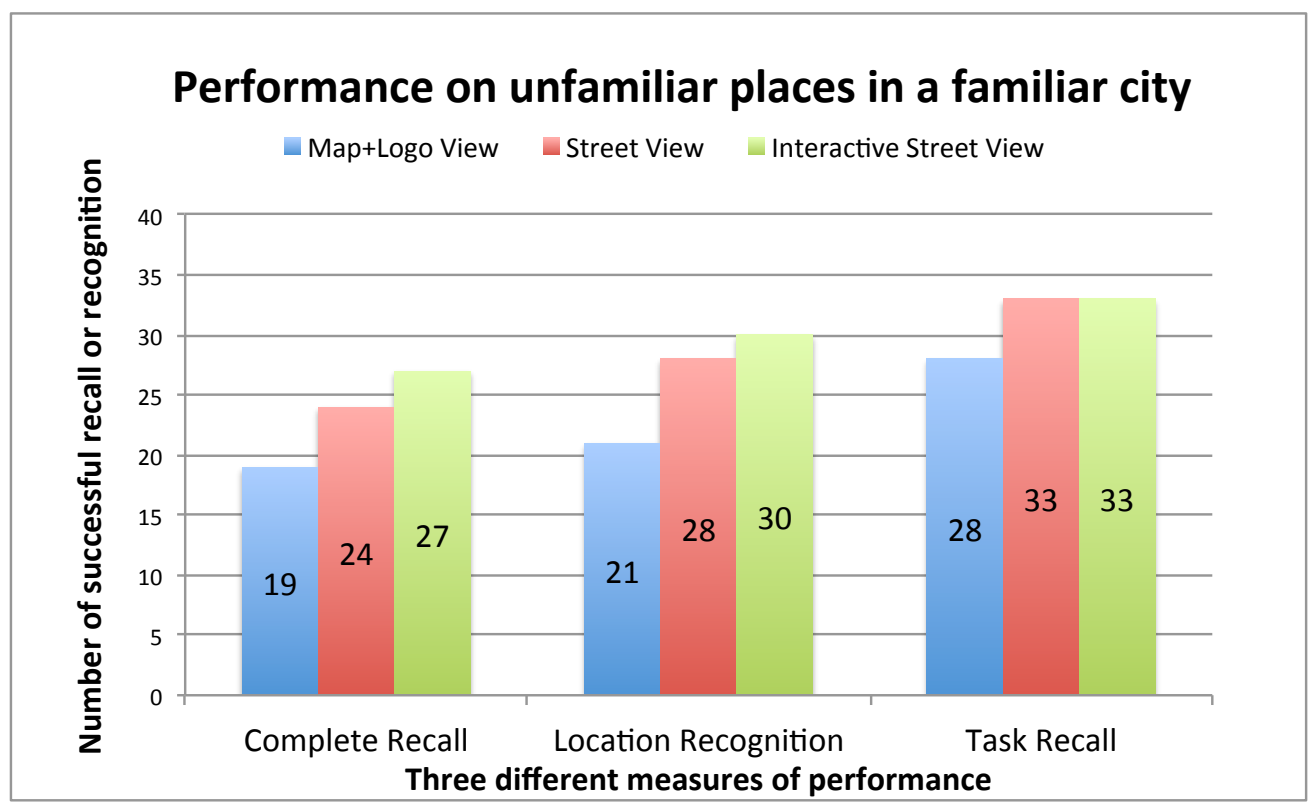

Figure 8.8: Performance of unfamiliar places in a familiar city for all three encoding methods.

\subsubsection{Familiarity of Places in an Unfamiliar City}

The final set of analyses compared the familiar places and unfamiliar places in the unfamiliar city. All participants in the traveling (unfamiliar) city rated each of the places (8) for familiarity using a 1-5 scale. The average familiarity ratings for each place is shown in Figure 8.9. The figure shows that five places (Office Depot, Wells Fargo, Walgreen's, FedEx, Target) were rated with an average rating above 4 (familiar). The other three places had an average rating under 4. Two places were local to the city of San Francisco (Academy of Art University, Peet's Coffee \& Tea). Furthermore, the closest Citibank branch is about 250 miles away, so Citibank was considered relatively unfamiliar to the participants in this study. 


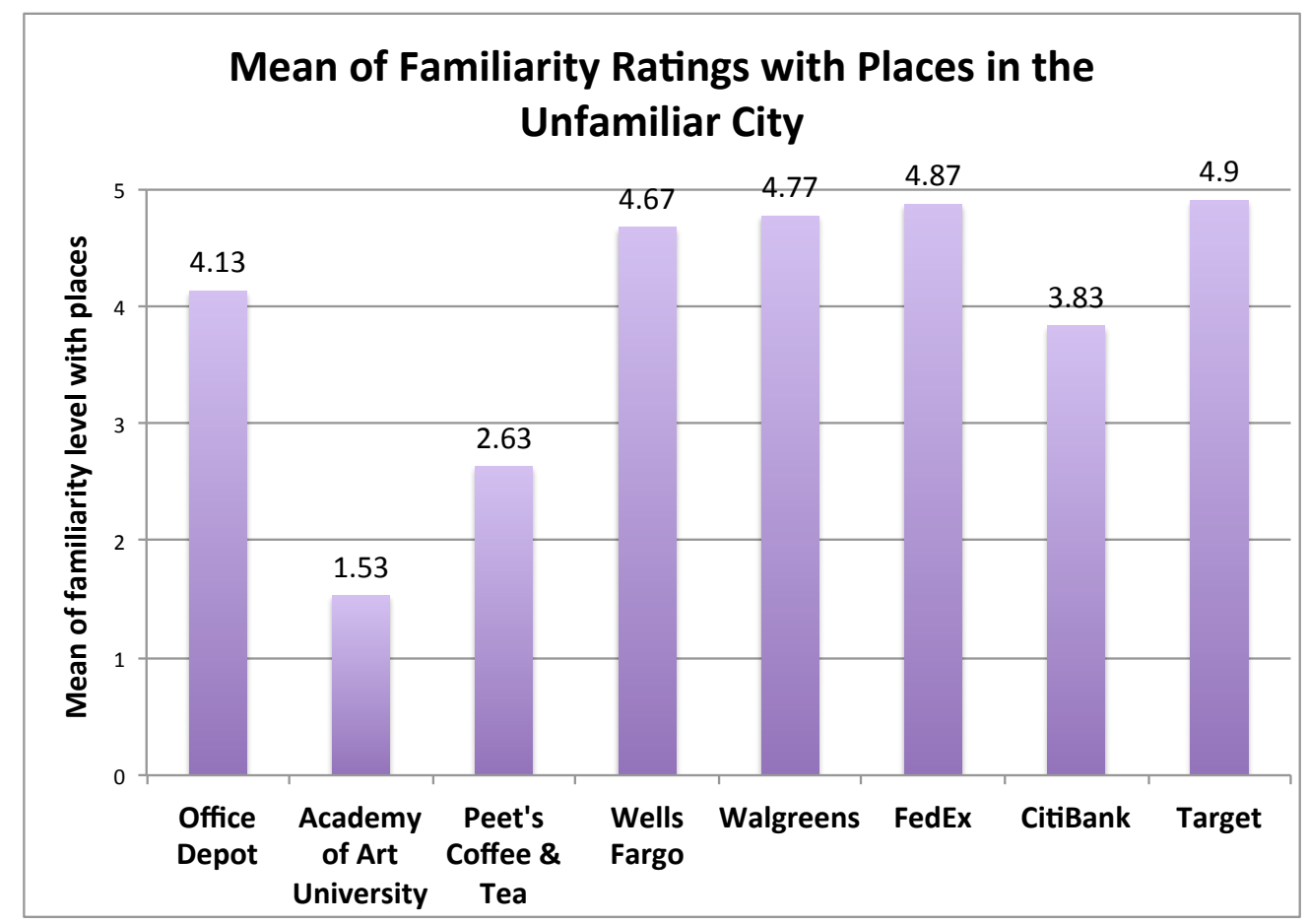

Figure 8.9: Average familiarity ratings with places in unfamiliar city

\section{Hypothesis 5: Familiar Places in an Unfamiliar City.}

Hypothesis 5: When people are familiar with a place in an unfamiliar city, performance (as measured in this study) improves with Street View because people would depend more on landmark knowledge than on procedural or survey knowledge due to their lack of familiarity with the city.

The five familiar places were rated at an average at 4.67 (out of 5). Using the five familiar places in the unfamiliar city, I performed three chi-square tests of independence to examine the relationship between the encoding methods and the performance under the three different measures. The performance under three measures is shown in Figure 8.10.

I found a significant difference on Complete Recall $\chi 2(2, N=150)=6.361, p=.042$ among the three encoding methods. Further paired chi-square analyses showed that participants 
using Street View performed significantly better (had more complete recalls) than those using Map+Logo View $(p=.012)$. No significant difference was found between Interactive Street View and Street View $(p=.271)$, nor between Interactive Street View and Map+Logo View $(p=.155)$.

I found a significant difference on Location Recognition $\chi 2(2, N=150)=10.345, p=.006$ among the three encoding methods. Participants using Street View performed significantly better (more locations recognized) than those using Map+Logo View $(p=.009)$. Participants with Interactive Street View also significantly outperformed than those using Map+Logo View $(p=.009)$.

I found a significant difference on Task Recall $\chi 2(2, N=150)=6.151, p=.046$ among the three encoding methods. Participants using the Street View performed significantly better (recalled more tasks) than those using the Map+Logo View $(p=.013)$. No significant difference was found between Interactive Street View and Street View $(\mathrm{p}=.096)$, nor between Interactive Street View and Map+Logo View $(p=.398)$.

\section{Increasing Location Familiarity in an Unfamiliar City}

I performed a logistic regression to ascertain the effect of familiarity with places on the likelihood that participants will successfully recall the task or recognize the location in the unfamiliar city. The logistic regression model was not statistically significant under Complete Recall $\chi 2(1)=.080, p=.777$, nor under Location Recognition $\chi 2(1)=2.260, p=.133$, nor under Task Recall $\chi 2(1)=2.556, p=.110$. The familiarity with places did not place a significant effect in predicting the performance in remembering location-based to-do tasks in the unfamiliar city.

The results show support for Hypothesis 5. Street View was a better interface (participant 


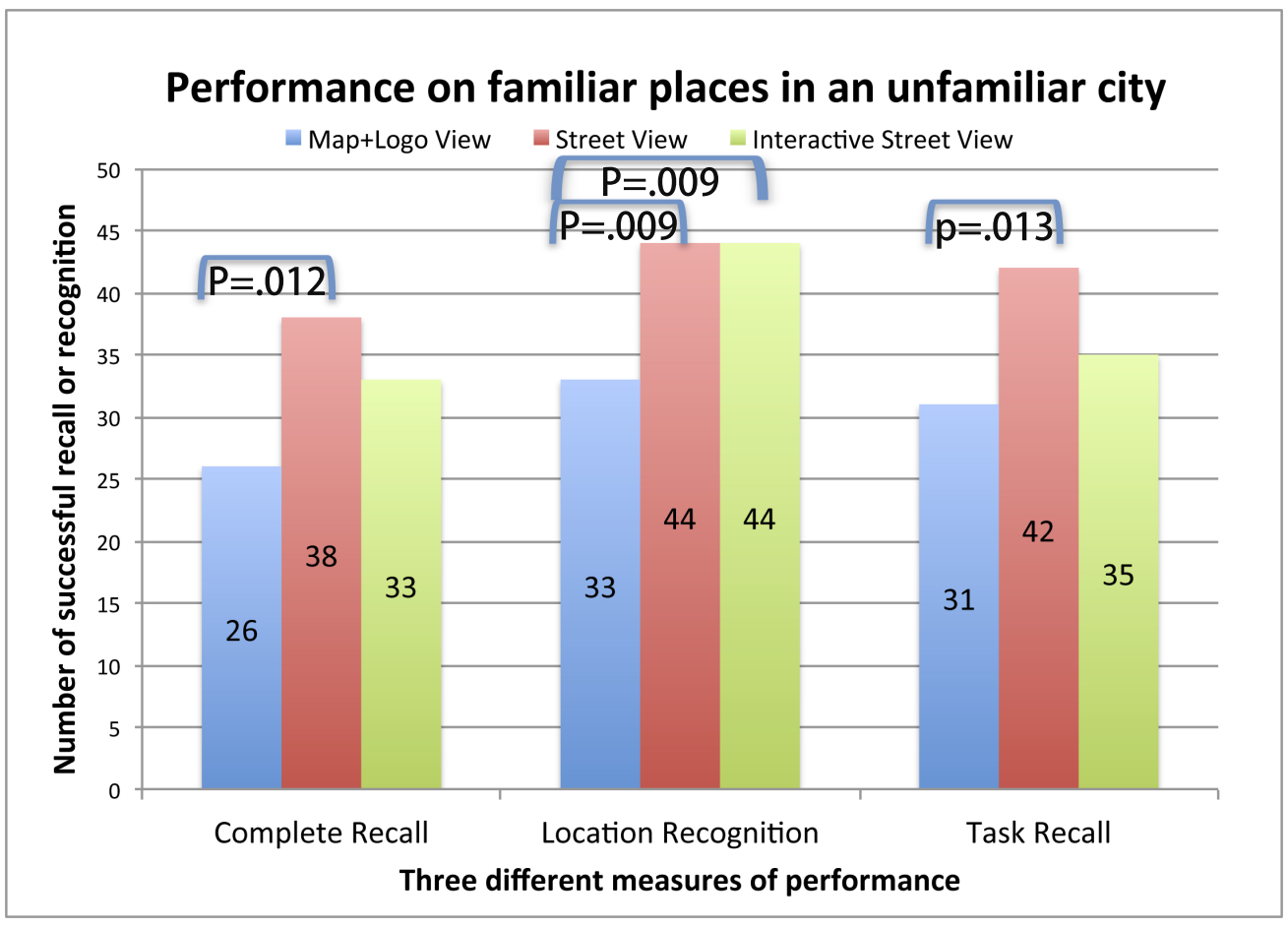

Figure 8.10: Performance of familiar places in an unfamiliar city for all three encoding methods.

had better performance using this interface) than the other two in supporting location recognition and task recall in an unfamiliar city. Due to the lack knowledge of the city, a perfect match (Street View) without requiring participants to shift between different types of spatial knowledge shows an advantage.

\section{Hypothesis 6: Unfamiliar Places in an Unfamiliar City.}

Hypothesis 6: When people are unfamiliar with a place in an unfamiliar city, performance (as measured in this study) improves with Street View and/or Interactive Street View because the interface provides additional contextual information (landmark and procedural knowledge).

The three unfamiliar places were rated at an average at 2.66 (out of 5). Using the three unfamiliar places in the unfamiliar city, I performed three chi-square tests of independence 
to examine the relationship between the encoding methods and the performance under the three different measures. The performance under three measures is shown in Figure 8.11.

I found a significant difference on Complete Recall $\chi 2(2, N=90)=6.299, p=.043$ among the three encoding methods. Further paired chi-square analyses showed that participants using Street View performed significantly better (had more complete recalls) than those using Map+Logo View $(p=.024)$. Participants with Street View also significantly outperformed those using Interactive Street View $(p=.024)$. No significant difference was found between Map+Logo View and between Interactive Street View $(p=1.000)$

I found a significant difference on Location Recognition $\chi 2(2, N=90)=9.317, p=.009$ among the three encoding methods. Further paired chi-square analyses showed that participants using Street View performed significantly better (had more complete recalls) than those using Interactive Street View $(p=.002)$. Interestingly, no significant difference was found between Street View and Map+Logo View $(p=.071)$.

I found no significant difference among three encoding methods on Task Recall $\chi 2(2, N=$ 90) $=1.869, p=.393$.

According to the result, Hypothesis 6 is partially confirmed, as Street View had a significantly better performance on Complete Recall and on Location Recognition but with the caveat that Interactive Street View was not in fact advantageous to memory performance.

\subsubsection{Interactive Street View}

To better understand the role of Interactive Street View on the performance in familiar and unfamiliar cities, I conducted exploratory analyses of the encoding time participants spent on memorizing the tasks, and their subjective comments at the recall stage. 


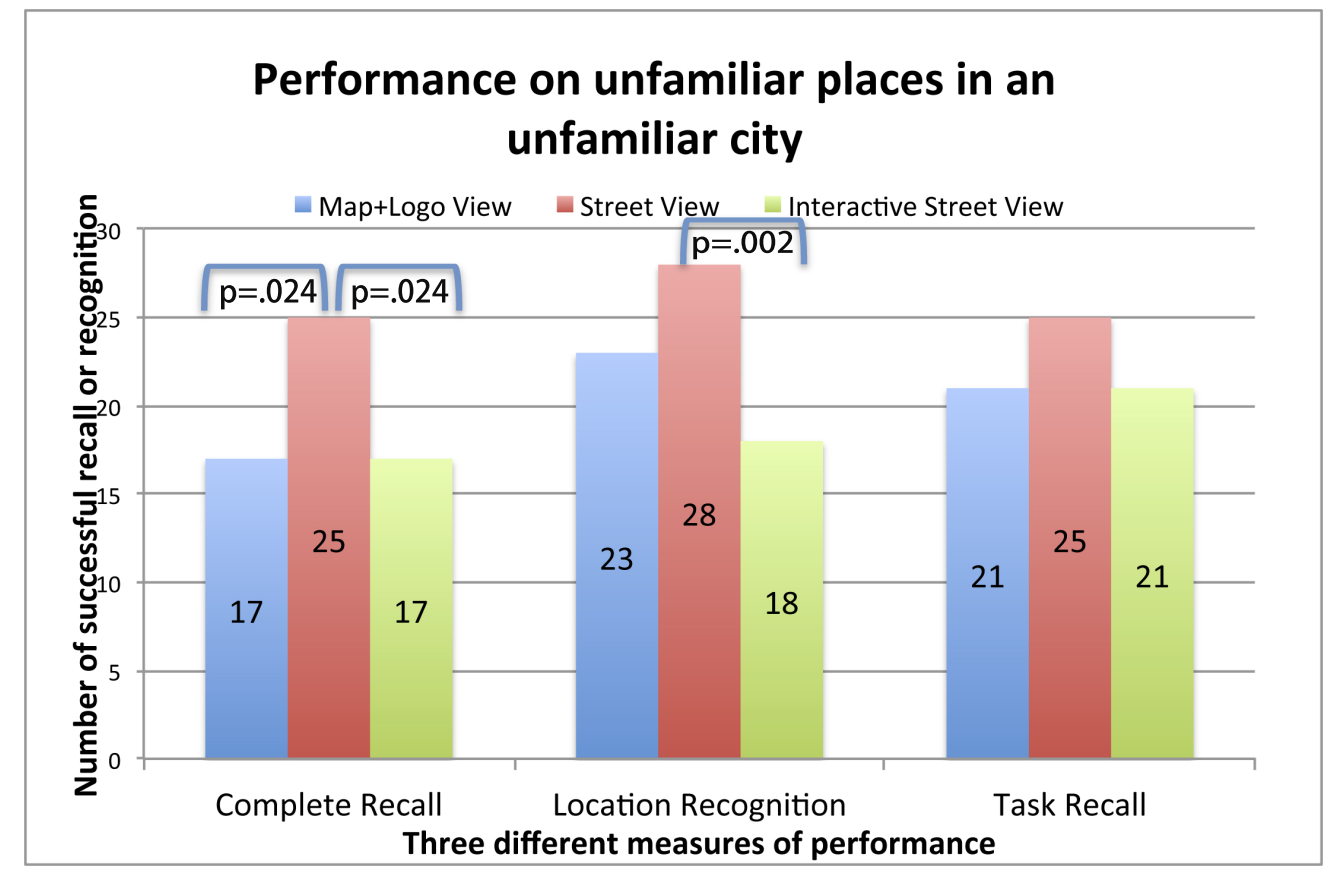

Figure 8.11: Performance of unfamiliar places in an unfamiliar city for all three encoding methods.

I conducted a two-way ANOVA on the influence of the two independent variables (encoding method, city familiarity) on encoding time. The encoding method included three levels (Map+Logo, Street, and Interactive Street) and city familiarity consisted of two levels (familiar/home, unfamiliar/traveling).

The main effect for city was not statistically significant $[F=1.482, p=.229]$, between familiar/home $(M=424.10, S D=173.008)$ and unfamiliar/traveling $(M=476.27, S D=$ 214.739). There was no statistically significant interaction effect between the two main factors, $[F(2,54)=.887, p=.418]$.

The main effect for encoding method yielded a significant $\mathrm{F}$ ratio of $[F=12.151, p=.000]$, indicating that the effect for encoding methods was significant. According to Tukey HSD results, participants with the Interactive Street View $(M=598.45, S D=212.799)$ spent significantly $(p=.000)$ longer time than those using the Map+Logo View $(M=360.40, S D=$ 
141.719) while memorizing the task (in Session 1). Furthermore, participants with the Interactive Street View spent significantly more time $(p=.001)$ than those encoding with a Street View $(M=391.70, S D=132.753)$. The comments provided by the participants of the two city groups point to different explanations of how Interactive Street view was used.

\section{Interactive Street View in a Familiar City}

In a familiar city, Interactive Street View was very helpful in remembering locations according to the participants' comments. For example, participant 1301 commented, "I tried to associate the location on the map that was shown with what I was doing. I think it was helpful, especially for the one street the post office was on. I don't really go on that street, that was helpful, look and see what was around it, and try to find something familiar, somewhere I can make that connection." Participant 1305 explained how s/he used the Interactive Street View as "For some places like the laundry shop, I tried to remember the CVS. And then for the fishing supplies, I tried to remember the Campus Automotive because I am not really familiar with the place but I know all the surroundings pretty well." Participant 1308 suggested that the surrounding buildings were helpful: "I think maybe the surrounding buildings (were useful), cause like for the signs, you can always read what it said. So probably the surroundings, like the hotel was next to the bank, and then for the Cleaner place, CVS was across the street. I recognized the CVS."

When participants encoded the places using Interactive Street View, they appeared to identify personally relevant familiar landmarks near the target location. For example, for the task to buy jewelry at Kent Jewelry, participant 1305 typed the task description in Session One as "Buy jewelry in front of Mellow Mushroom(Kent Jewelry)". Mellow Mushroom Pizza is a popular restaurant across the street from Kent Jewelry. In another example, for

the task to pick up laundry from A Cleaner World, participant 1302 typed the task as "Col- 
lect laundered suits from a cleaner world near IMAX." In these two examples, they encoded their unfamiliar business (Kent Jewelry and A Cleaner World) using familiar places (Mellow Mushroom and the IMAX theater).

\section{Interactive Street View in an Unfamiliar City}

In the unfamiliar city, however, Interactive Street view received negative comments, as it appeared to be confusing some participants. For example, participant 2304 commented that "No, I didn't use it (Interactive Street View) at all, I didn't find it helpful cause I couldn't

figure out, I couldn't orient myself at all, like it was, it confused me a little bit, like I didn't know where to look." Participant 2307 reported the following with respect to the Interactive Street View: "I mean it could be a little easier, it was kind of difficult to going all around it. Ah..I would accidentally click on the bottom right area, and it would take me to another like 2 or 3 meters, and I would be like 'Where am I now?."

Some participants expressed preference for multiple static images capturing some of the surroundings but without the complexity of the interaction. For example, participant 2307 suggested that "I think multiple pictures like from different angles, and some of the buildings around it, maybe an initial video (...). But the Google Map thing is still good, it's fine, I am just saying maybe pictures will be better." Participant 2309 made a similar comment: "I think for me it would be more helpful if I would see pictures, just like still pictures rather than interactive and moving around."

Still, the surroundings helped participants to build their landmark knowledge. For example, for the task to get quarters from Wells Fargo, participant 2302 typed the task as "laundry quarters from wells fargo. cali st. american flag and cali flag near to the wells fargo building. two point perception view of buildings.baroc arch building for us bank across.trolley railway." 
This participant typed a lot of landmark information surrounding the bank.

\subsubsection{Topographical Features of the City}

The two cities I used in the experiment not only have different familiarity levels, but they also have different topographical features. Blacksburg is a small town with typical two-lane streets, while San Francisco has a grid plan with wider streets. I found that participants' space perceptions were quite different in these two cities. For example, participant 2210 commented: "In San Francisco, it's more blocks. So generally you ask people where is it, they'll just say three blocks. But here, it's like get on this way or that way, Blacksburg is a little less structured than the city." Also, participant 2305 reported, "San Francisco, a lot more things I feel like look alike, there is a lot more around you. But in Blacksburg, there is not as many alike (...), everything is pretty unique, very easy."

\subsection{Design Implications}

\subsubsection{Implications for LBRs "At Home"}

Participants encoding locations in the home city performed well in all interfaces. I found no significant difference in performance (on any of the measures) in this group. I did find, however, that increased familiarity with a place increases the likelihood of recognition for that place, as expected. Participants in this group found the Interactive Street View useful (based on comments) and were able to rotate the camera to identify familiar elements in the

environment that helped them recognize unfamiliar places. However, that ability did not have a significant effect on the performance. 
However, since encoding a task using Interactive Street View took longer than using the other two conditions, I suggest using a simple and intuitive interface at the encoding stage for familiar city. Encoding a map view of the place with a distinctive business logo might provide enough support in recognizing the cue at the retrieval stage.

\subsubsection{Implications for LBRs "While Traveling"}

Participants in an unfamiliar city, however, saw significant increase in performance when using the Street View interface. I expected the Interactive version of the interface to produce better results. I assumed that being able to look around, zoom in and out, and even move in the space would be a scaffold for participants in an unfamiliar city. However, that is not what I found. Street View produced a better performance almost unanimously for location recognition as compared to the interactive version.

Based on participants' comments, Interactive Street View seemed to be confusing for the participants. At first I found this to be counter-intuitive to our expectation. However, upon closer inspection, I inferred that participants who are unfamiliar with the city or the location may become disoriented by interactive viewpoints. After moving the street view image, participants were disoriented and did not know how to re-position themselves in a way that was beneficial for navigation.

\subsubsection{Familiarity with City More Impactful Than Place}

Considering the results, I found that familiarity with a place can be put at a second-place compared with the effect of familiarity with a city. As long as the place was located in a familiar city, no significant difference was found between the three encoding methods. Even if it is an unfamiliar place, participants could easily learn the information of the target location 
provided by Map+Logo view because of the rich mental map they already possessed about the city. People could correctly identify the road names and the surrounding contextual information through a quick glance at the encoding interface. They could even set up their personal-relevant familiar landmarks as external cues from the map interface. In this sense, providing a map view of the place in a familiar city makes no difference with providing a street view, which is consistent with our results. I assume that very little effort is required for participants to translate between the egocentric view and allocentric view in a familiar city. Additionally, the logo provided as a perceptual icon at the encoding stage serves as an effective external cue at the retrieval stage. Most participants in the Map+Logo View group commented that the visual logo was very helpful to recognize the location.

On the other hand, as long as the place is located in an unfamiliar city, Street View had a significant effect on improving performance on location recognition. Even the place is a relative familiar business, participants still failed to acquire enough visual information of the target location from a map view at the encoding stage due to the lack of knowledge with contexts. This requires that participants transform the location information from survey knowledge to landmark knowledge all by themselves at the retrieval stage. In an unfamiliar city, providing enough landmark knowledge and procedure knowledge is necessary to support a successful external cue recognition.

\subsubsection{Adaptation of interface according to familiarity}

As user's length of stay in a location grows, they go from unfamiliar to more familiar with a city. Computationally and location-enabled devices should be able to capture, process and adapt interfaces based on the growth of familiarity of a location. Getting directions, for example, to a business location that is just a block away from our office, should not entail 
a full set of directions from home. Instead, mapping services could simply provide relative directions starting from a known landmark and using relative routes.

\subsection{Summary}

This study offers implications for the design of LBRs, in particular for how LBRs should consider the user's familiarity level with the places and adapt the interface to provide a better user experience. In a familiar location context, the business logo given as a perceptual icon at the encoding stage serves as an effective external cue at the retrieval stage. I suggest that LBRs should display the map view of the target location during encoding, with an additional distinctive business logo for cities that are familiar to the users. While in an unfamiliar location context, I suggest that LBRs should display one or multiple street-view pictures of the target location during encoding for cities that are unfamiliar to the users. These pictures should provide enough spatial knowledge for people successfully recognizing the location while navigating in the unfamiliar environment. 


\section{Chapter 9}

\section{LBR Prototype Design and}

\section{Evaluation}

This chapter describes the design of an LBR prototype and reports the results of a usability evaluation study on this prototype. This prototype incorporates the lessons learned in the user studies presented in this dissertation.

\subsection{Introduction}

In this chapter, I applied the findings of my user research studies to the design of a LBR prototype. In chapter 7, I demonstrated that seeing a Street View at the encoding stage has a significant effect on supporting people's recognition of locations and recall of associated tasks. Interactive Street View provides additional spatial information for people to learn the location, so this prototype encoding interface was designed using an Interactive Street View. That is, when users select a location to complete a to-do task, they see the Interactive Street View of that location instead of the Map View (the typical current designs). 
This LBR prototype was also designed based on different types of locations. It allows users to select a particular location or a generic location for completing a to-do task. In chapter 5, I found that location information is used differently for completing different types of to-do tasks. For example, "pick up milk" is a task that can be done at any grocery store, whereas "return a book" is a task that has to be completed at the particular library where the book was borrowed.

The task scenarios of this prototype evaluation were about different conditions to see how familiarity with the places influences people setting up the reminder. One of my empirical user study found that familiarity with the city and the business location has a significant impact on people recognizing that location (Chapter 8). In this evaluation setup, the three task scenarios were designed under different conditions: task 1 was a task to be done at a familiar business in a familiar city, task 2 was a task that has to be set up at an unfamiliar business in an unfamiliar city, and task 3 was to be done at a familiar business in an unfamiliar city.

\subsection{Iterative Design of Prototype}

I first created a paper prototype to get a general idea of using LBR to set up a reminder. After receiving feedback from a few pilot studies, the design was narrowed to three new features: Interactive Street View, different types of locations and different levels of familiarity with places. I then created wire-frames and designed a low-fidelity prototype using POP to include these new features in an LBR. This LBR prototype only focused on the implementation of new features. Finally, I redesigned the LBR encoding interface following iOS Reminders. The prototype encoding interface looks similar to current setup interface of iOS Reminders.

The high-fidelity prototype was developed on Xcode 8.2.1 in MacOS Sierra and run on an 
iPhone 7 Plus with an iOS 10.3.1. It supports two main functions: to allow users to set up a reminder at generic location or at particular location, and to allow users to see an Interactive Street View when encoding a particular location.

\subsubsection{Setup Reminder at Generic Location}

The main page of this LBR prototype shows a list of active reminders (Figure 9.1). First, the "+" button allows users to add a new reminder. Users can set up the reminder to be delivered on a day or at a place after entering what they want to do. Figure 9.2 is an example showing users setting up a reminder to "pick up milk." Specifically, my work focuses on how users set up reminders to be triggered at locations, so the function of "Remind me on a day" is not developed yet. The purpose of displaying this feature is to see how users understand this functionality.

Once the user selects "Remind me at a location", one of the two types of locations can be selected: Setup Particular Location (e.g. Kroger) or Setup Generic Location (e.g. grocery store) (See Chapter 5.3). Users can get milk from any grocery store, so "Setup Generic Location" is selected. Then users can search the location using the search bar. Figure 9.3 shows that users are searching nearby grocery stores. Then, the prototype displays a map view with several pins showing the nearby available grocery stores (Figure 9.4).

Once users click on the pin, it shows the business name of that particular location. Users can select and deselect any location showing on the map by clicking the pin. Figure 9.5 shows "Food Lion" is selected by the user as a place to trigger this reminder. Figure 9.6 shows that the user is adding one more location (Kroger) to trigger this reminder. Clicking the "save" button on the top right corner completes the setup of this reminder. The interface returns to the main page showing a list of active location-based reminders (Figure 9.7). 


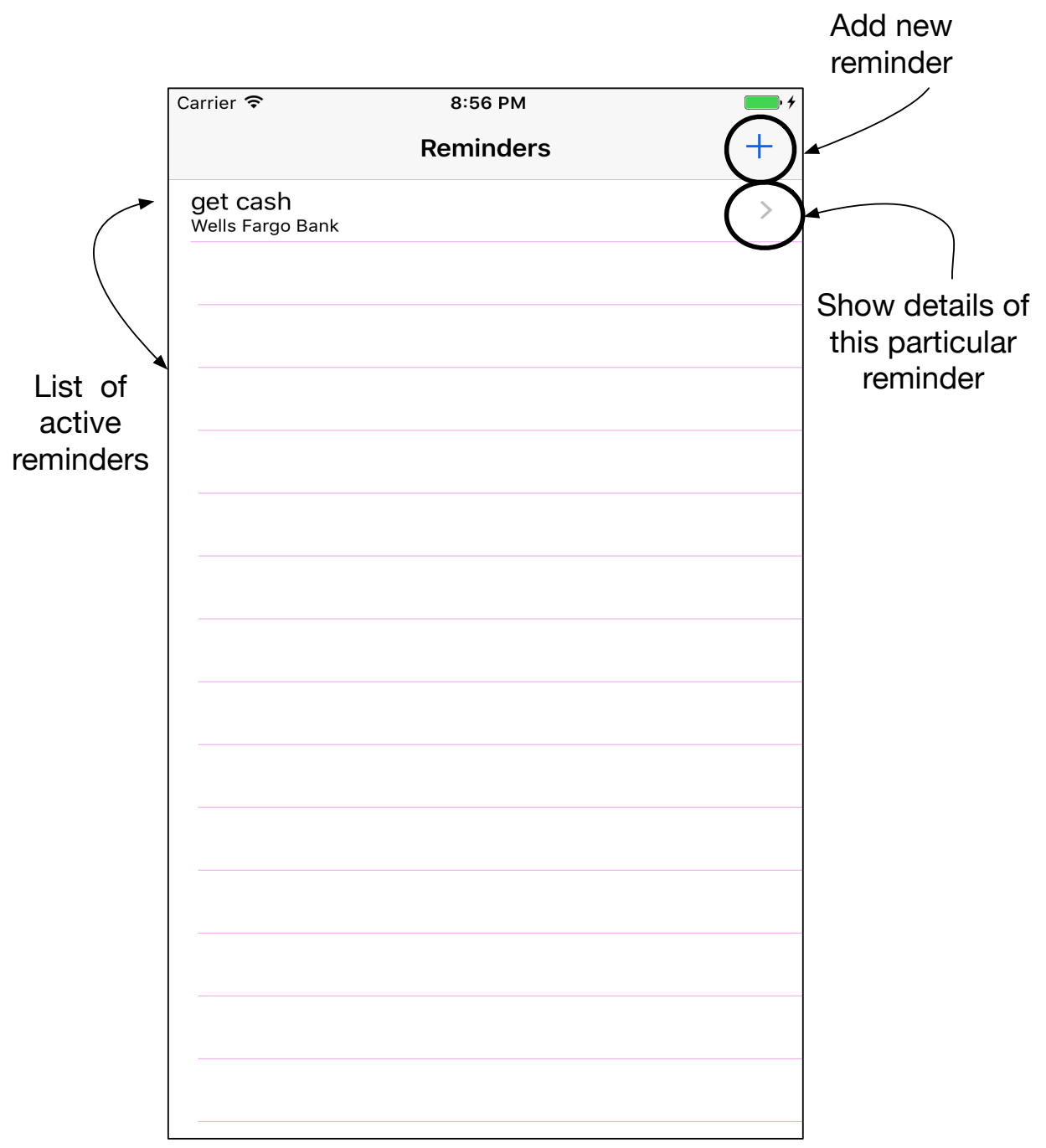

Figure 9.1: LBR prototype showing the active reminders 


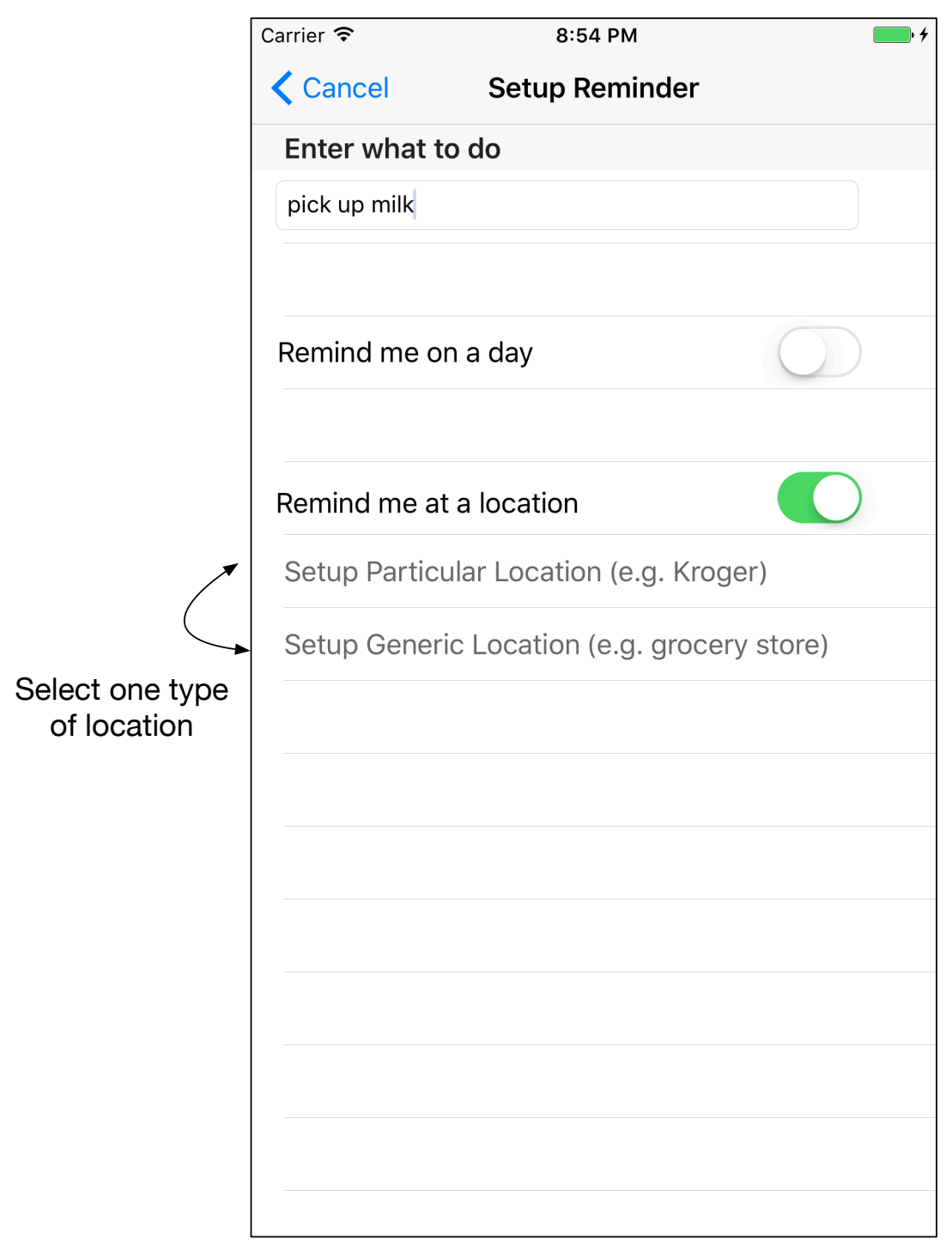

Figure 9.2: LBR prototype showing the feature of setting up a reminder 


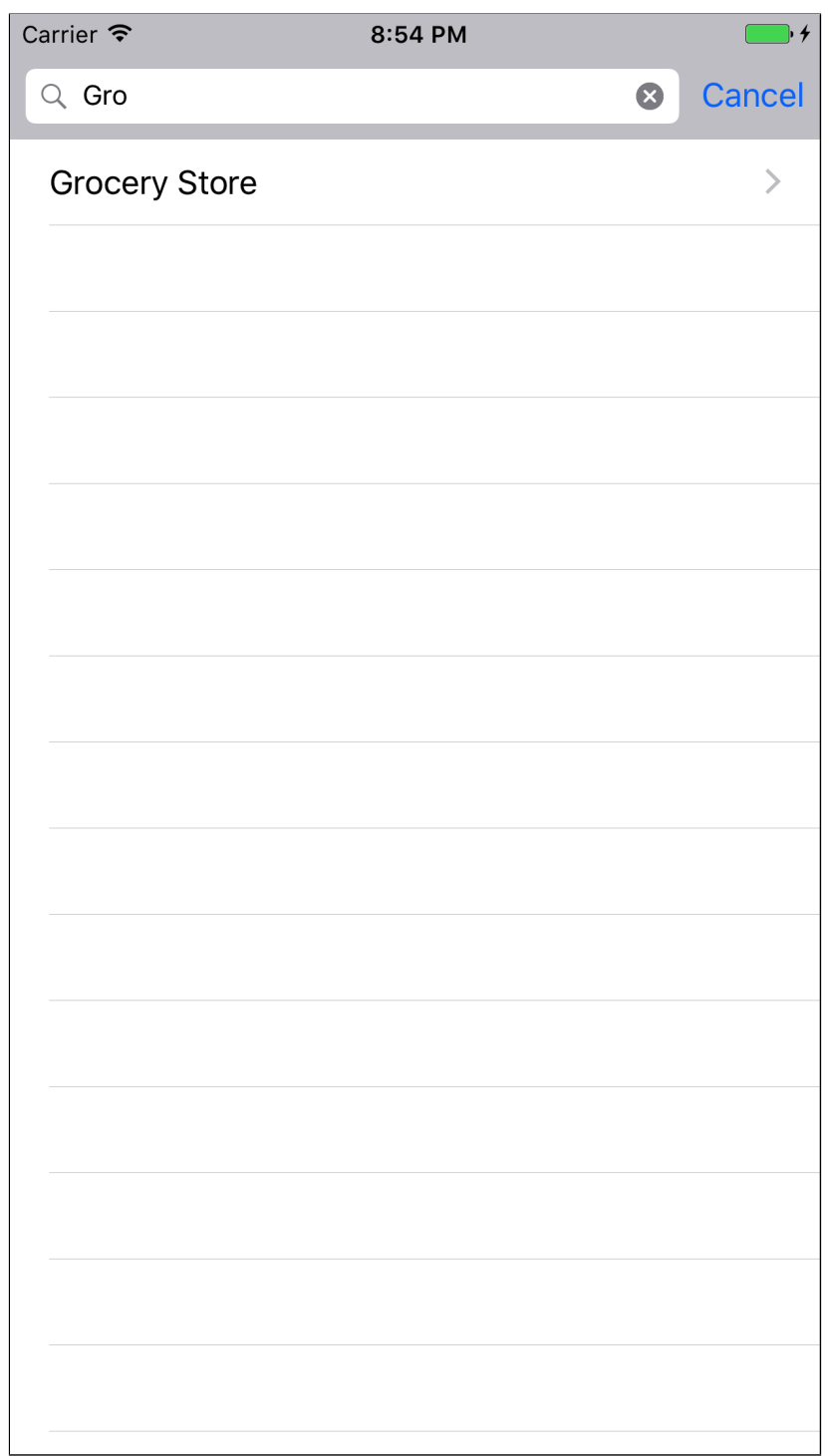

Figure 9.3: LBR prototype showing the feature of searching for a place 


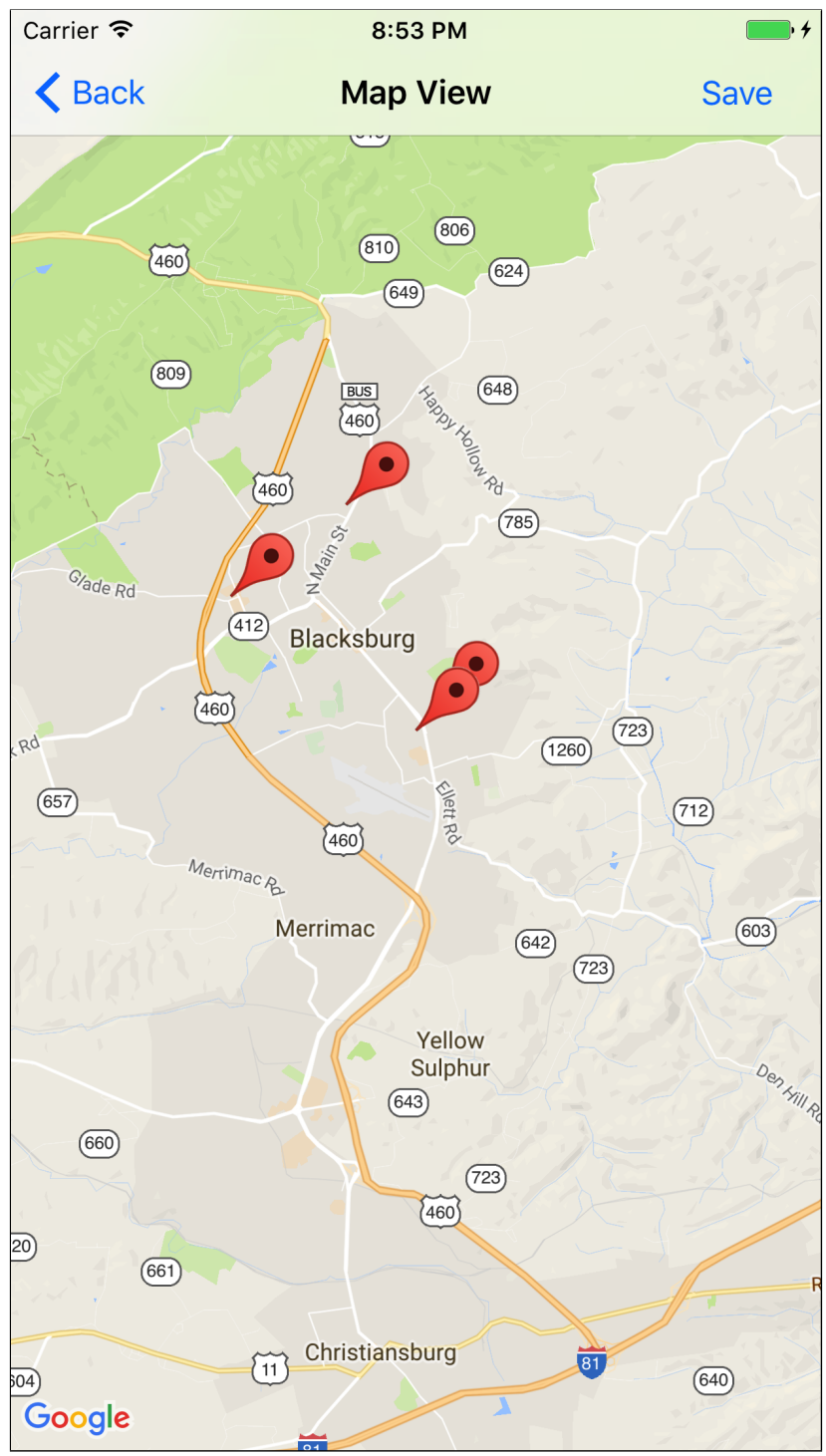

Figure 9.4: LBR prototype showing the map view with a list of available locations, from top to the bottom: Food Lion, Kroger on university city blvd, Oasis, Kroger on south main 


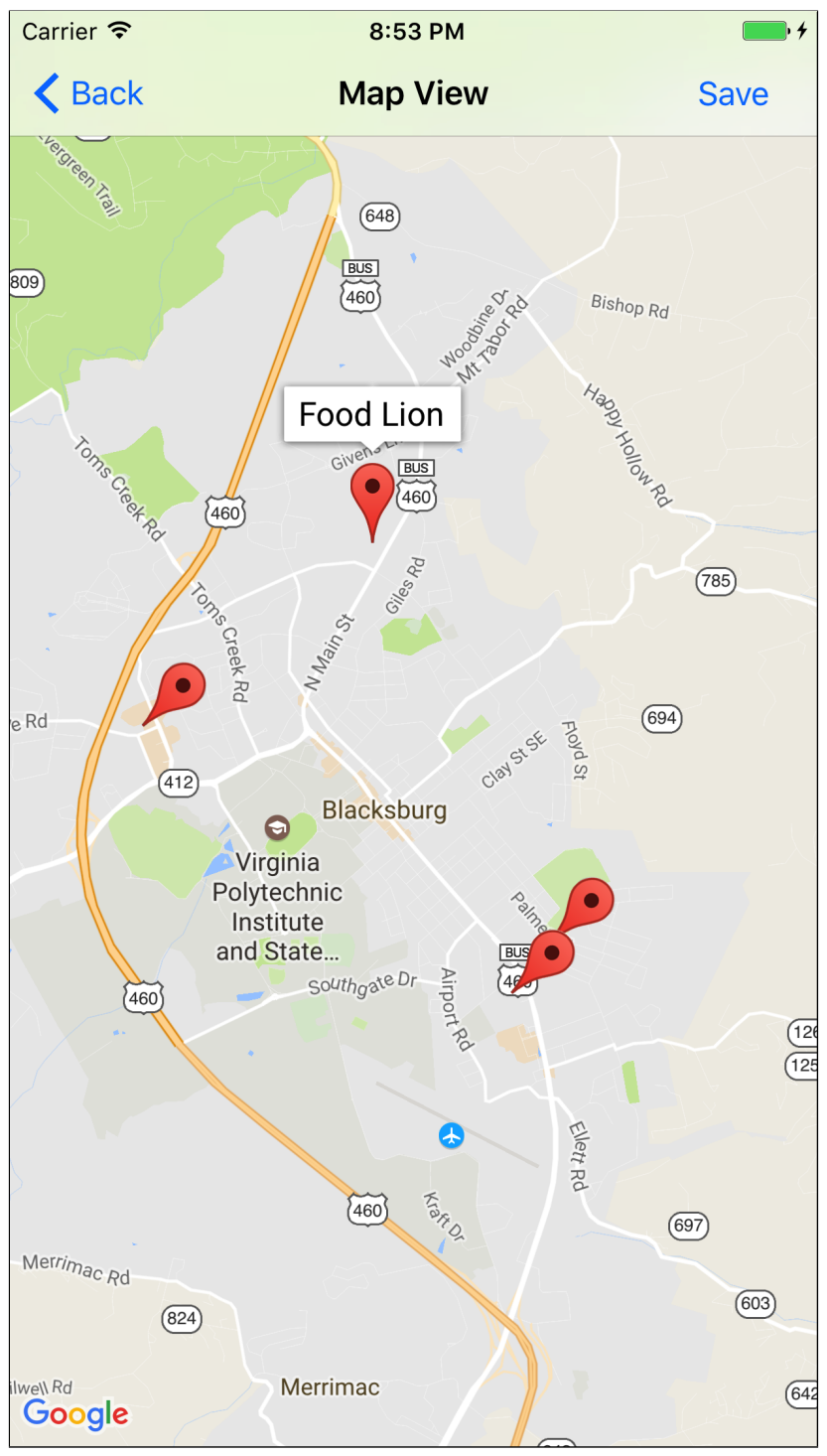

Figure 9.5: LBR prototype showing one location is selected by users 


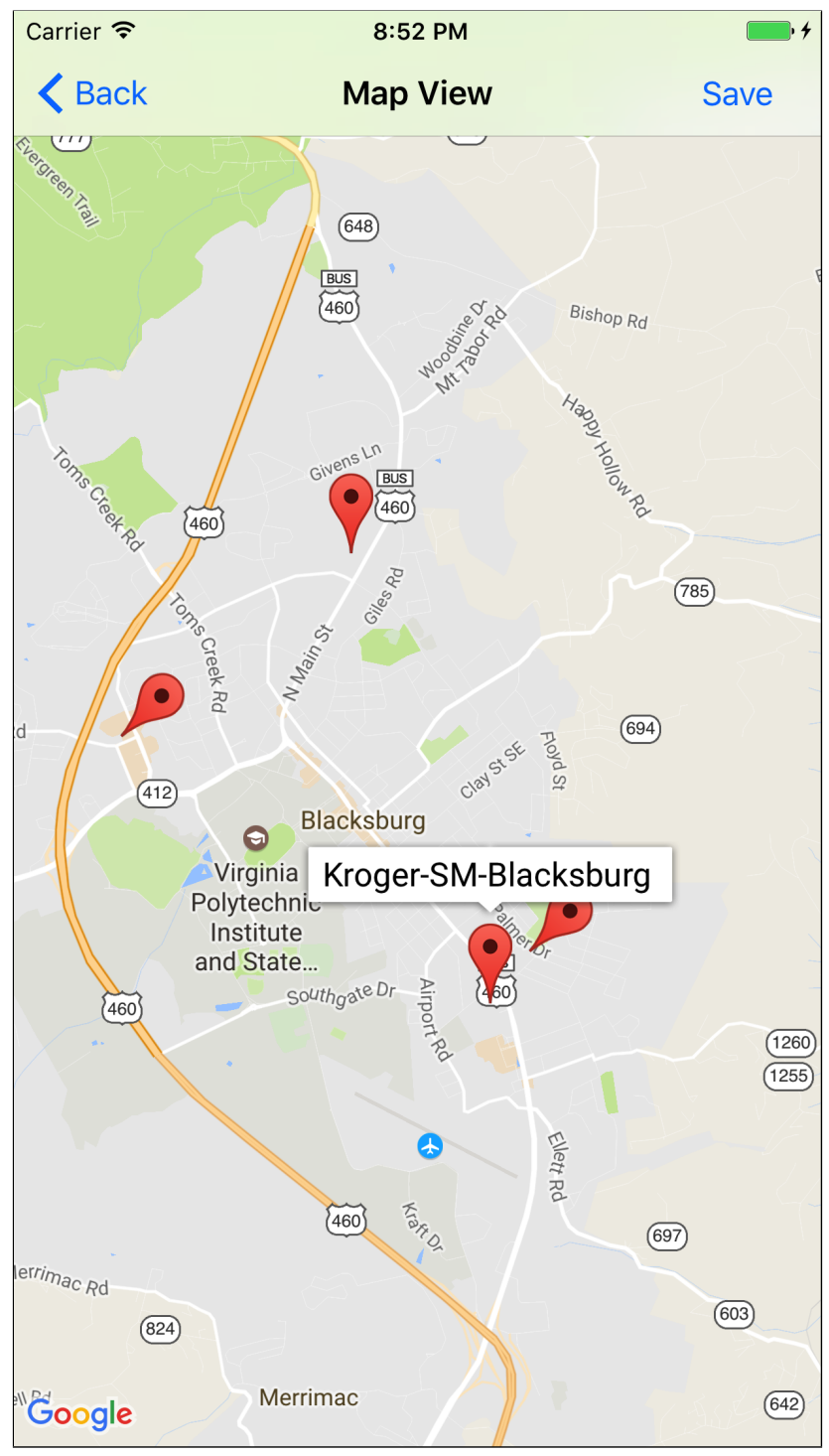

Figure 9.6: LBR prototype showing two locations are selected by users 


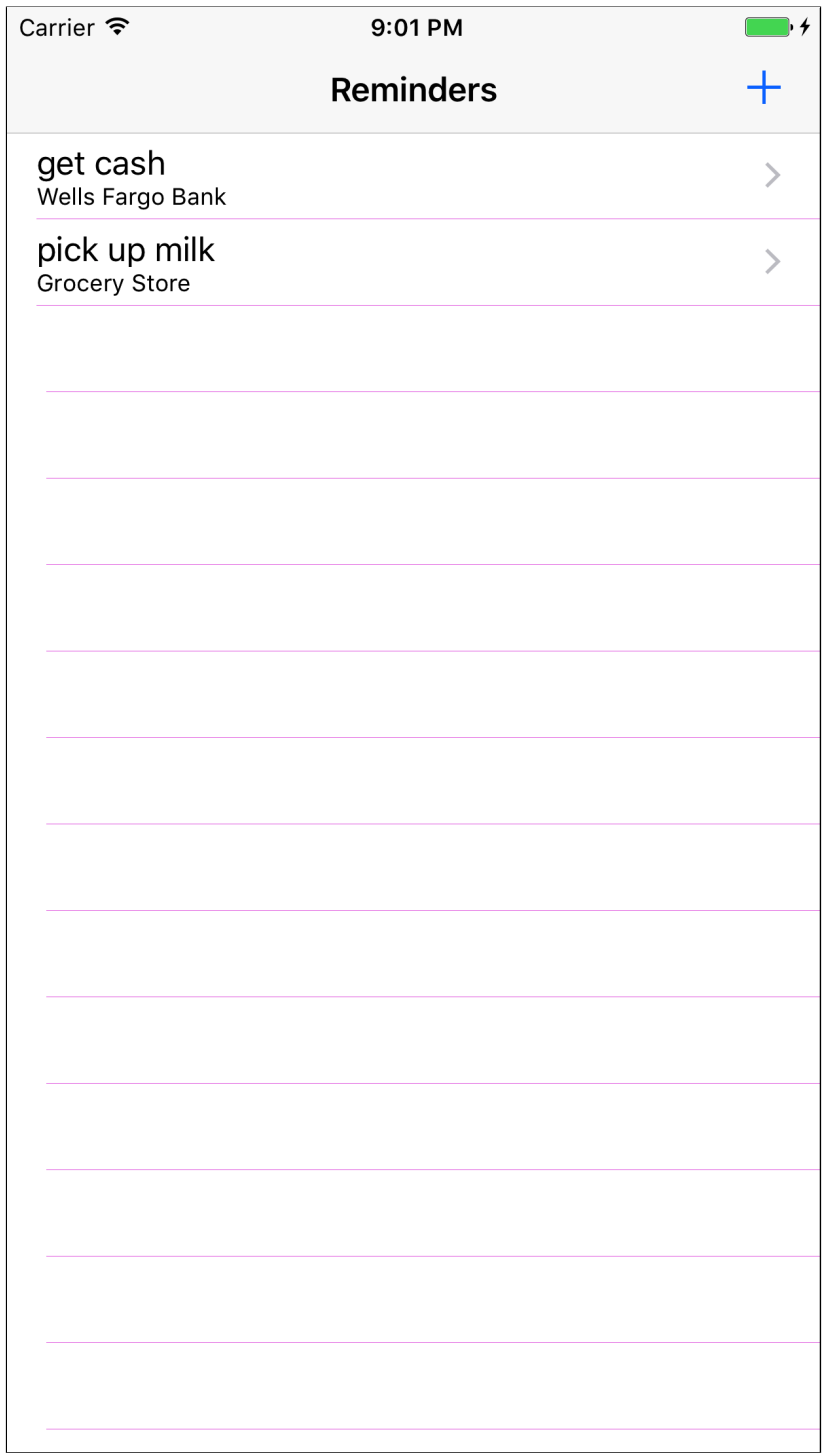

Figure 9.7: LBR prototype showing the completion of adding a new reminder 


\subsubsection{Setup Reminder at Particular Location}

Users also can create the reminder to be triggered at a particular location. For example, the reminder of "return a book to the library" needs to be delivered at a specific location (see Figure 9.8). In this case, the library must be a particular one where the book was borrowed. Figure 9.9 shows that users can search the particular location using the search bar. Then a Google Street View pops up showing the real-time Interactive Street View of this specified location (shown as Figure 9.10). Users are able to play with it by zooming in and out, moving forward and backward, as well as orienting themselves. Figure 9.11 shows the completion of adding this new reminder. Users are expected to learn more contextual information about the target location by seeing this Interactive Street View.

\subsection{Usability Evaluation}

The purpose of this LBR prototype usability study was to evaluate how people use an Interactive Street View and use different location types to set up a location-based reminder. The user study was conducted in a usability laboratory, which is McBryde Hall 133. This evaluation study was approved by the Virginia Tech Institutional Review Board with approval \# 17-195 on March 20, 2017.

\subsubsection{Participants}

I recruited six participants (four males and two females) from the SONA system in the Psychology Department and from a graduate students list-serve. The participants received

either course credits or SONA credits for their participation. Their ages are in the range of 19 to 27 years old. Their majors are from the computer science, psychology or biology 


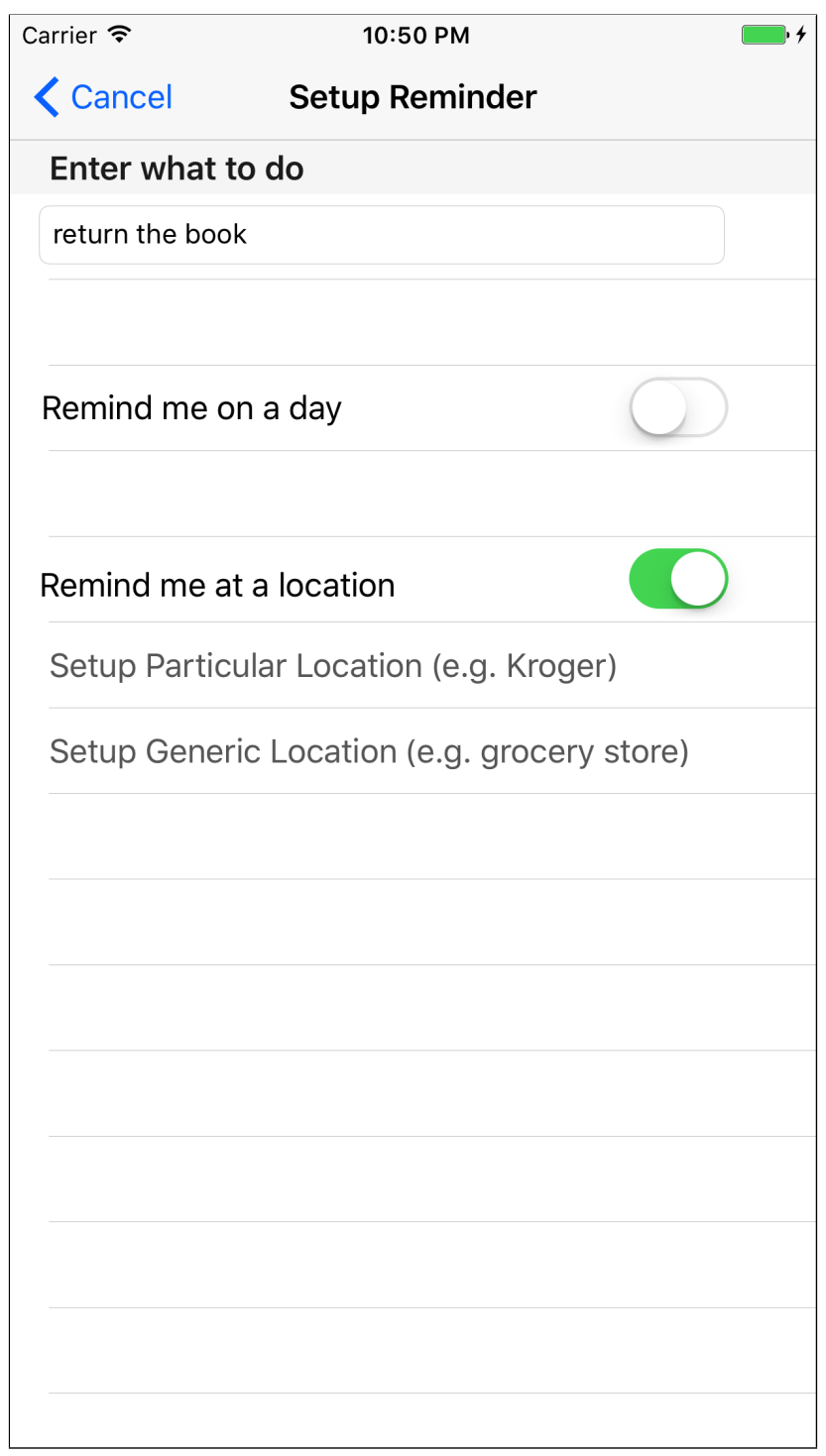

Figure 9.8: LBR prototype showing the feature of setting up a reminder 


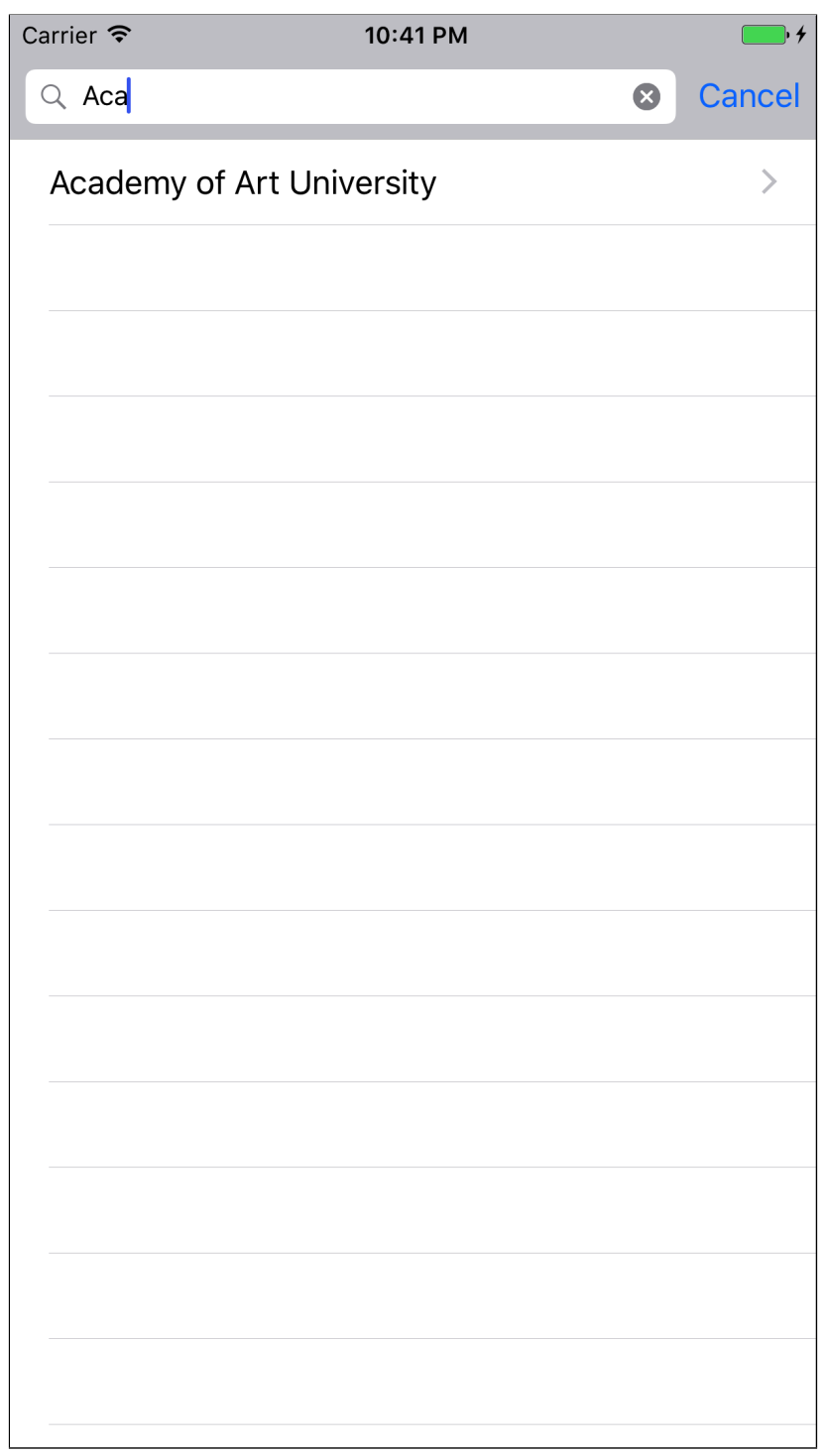

Figure 9.9: LBR prototype showing the search function for a particular location 


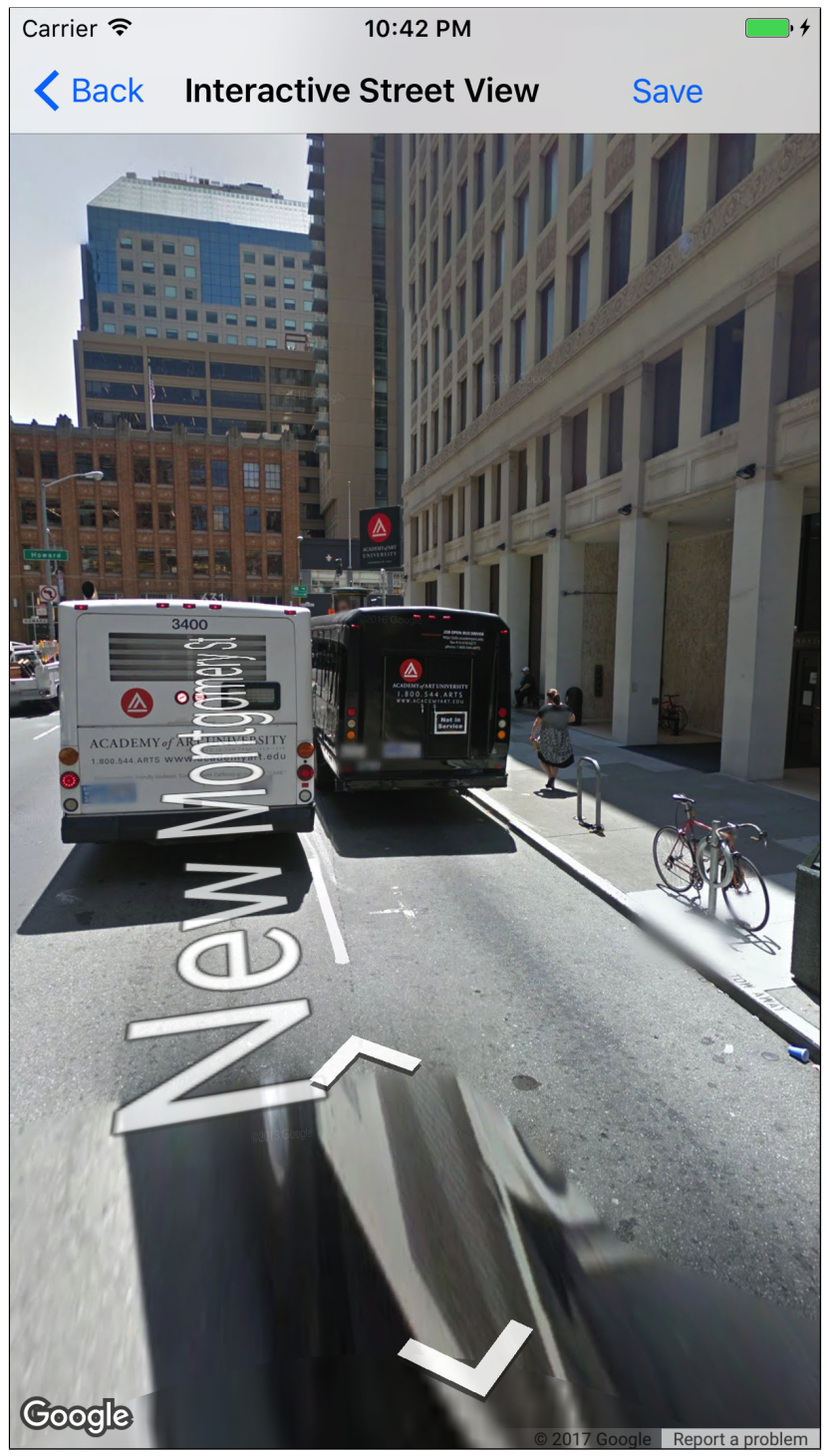

Figure 9.10: LBR prototype showing the Google Street View of a particular location 


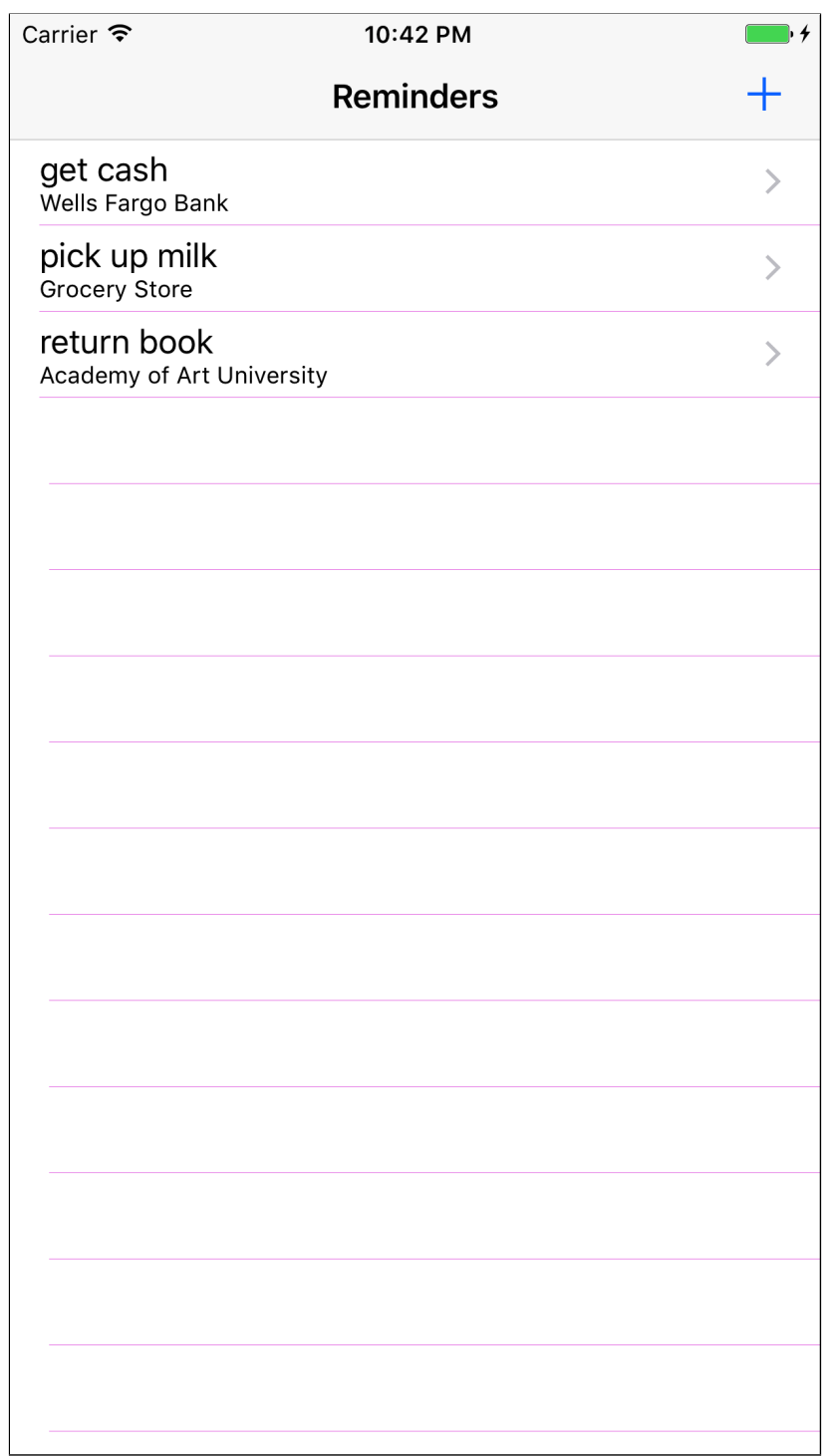

Figure 9.11: LBR prototype showing the completion of adding a new reminder 
departments. All of them had used the iPhone iOS system for two to six years. Five participants had used or were using Reminders on the iOS platform, and one participant had never used it before.

\subsubsection{Data Collection}

I recorded participants' subjective comments and the phone screens. Think-aloud protocol was used to understand users' thoughts while they performed the tasks. I conducted a short interview at the end of the study to collect users' using experiences.

\subsubsection{Task Scenarios}

I asked all of participants to use the given LBR prototype on a given iPhone (my iPhone) to set up three reminders in the same sequence. The three task scenarios are listed below:

1. Your roommate just texted you a message and asked you to pick up some milk from the grocery store. Several grocery stores are on your way home. To remember to complete the task, you decide to create a reminder for picking up milk using the given prototype. Therefore, the reminder can be delivered when you are near a grocery store on the way home.

2. One of your friends is studying at Academy of Art University at San Francisco. You are invited to visit her during the spring break. You want to pick up a piece of new art design from their school library before leaving, so you set up a reminder to remind you to pick up the design product while you are near the school.

3. You are driving to Charlotte to visit a family member. You have ordered a pizza for dinner from the Mellow Mushroom in the downtown area. You set up a reminder for 
picking up the pizza when near the Mellow Mushroom.

\subsection{Results}

\subsubsection{Subjective Comments}

\section{Participants liked to see street information}

Participants' comments confirmed that street view supports people recognizing the location better. All of the participants thought seeing an Interactive Street View was interesting and useful, since it could be helpful to learn the location. Participant 1 thought: "seeing street view might be kind of cool because people will remember the location by seeing the picture." Participant 2 thought street view was especially useful for "areas have block signs and trees, and when the building is kind of hidden." Participant 6 thought he might easily get lost without knowing what the building looks like: "I do like having the surroundings once I know the location, then when I am physically near that location I can figure out oh I am pretty close to it. If I didn't know how the building looked, I probably get lost on this street and I probably like pass this building because the sign is really small." Participants felt like Street View is more powerful for new locations than locations they had been to before. Participant 3 would like to use Street View to see "what it (target location) looks like, if I am unsure about the location or something, or it's a particular new environment." 


\subsubsection{Usability Issues}

\section{Participants get confused with location types}

Based on the use of the prototype, participants commented that they liked the feature of selecting location types. However, participants were confused with selecting a location type from generic location and particular location when they performed the task. For setting up the task "to pick up milk from grocery store", three participants first selected "Setup Particular Location" because they usually did this task at Kroger. Participant 1 was not sure of the meaning of the generic location. Participant 2 had a hard time deciding which one to choose: "Particular location and generic location is a bit confusing. It's kind of hard to differentiate what the two would have. When I need to go to grocery store, I wasn't sure... do I click just Kroger or any grocery store? It's a little bit confusing." Participants considered having two options as unnecessary: "I am not sure that the specified functionality that necessarily needs to be there" (Participant 1). Participant 6 commented, "I don't know what the particular location does because I can find particular store from a generic list." Both options have a search bar, so participants expected to search either "restaurants" or for a particular restaurant, rather than selecting a particular type of location on the setup page. Participant 5 considered that having one search bar would be much easier: "if you search Kroger, all Kroger pop up, if search grocery store, all grocery stores pop up".

\section{Participants get lost using Interactive Street View}

Participants easily get lost while using the Interactive Street View. Participant 1 commented: "Interactive Street View, that's interesting. But it doesn't say library. So am I supposed to set this up? I was expecting an option for school library...Is this the library? The label didn't say library." Participant 2 thought Street View was "maybe a little bit disorienting. 
You just appear there, you don't have much to reference about where you are." Participant 6 was not sure how to respond to the Street View: "This is pretty cool, but I don't know what I am looking at."

\subsection{Design Implications}

Overall, showing an Interactive Street View can be very useful in supporting participants to learn the surroundings about the location. Participants considered that seeing visual items could be helpful in remembering and recognizing the location at a later time. However, participants easily get lost using the interactive feature. Participant 3 also commented, "I would expect Street View as a drop in, but I wouldn't expect it as the main screen cause it doesn't tell me that I actually am where." Participants like to have the Street View as an extra support showing contextual information about the location, at the same time they like to see a Map View first to have an idea of where the location is. Participant 6 considered map information "as a confirmation, that this is the location I want, cause what if there is multiple Academy of Art University?"

Future LBR designers should consider incorporating street view into the encoding interface with distinctive cues showing the target location. Interactive Street View is not enough as it does not tie the world to the task itself, maybe some pop ups are needed for future LBR encoding interfaces. To be more specific, LBR designers should combine map data with street information to provide effective reminder setup at the encoding stage. Map data gives users enough route information and the global spatial relations with surroundings, while street view serves as an extra help to provide more contextual information and visual items about the target location. Figure 9.12 shows a suggested encoding interface for future LBRs.

Furthermore, LBR designers should support people to complete a task at different types of 


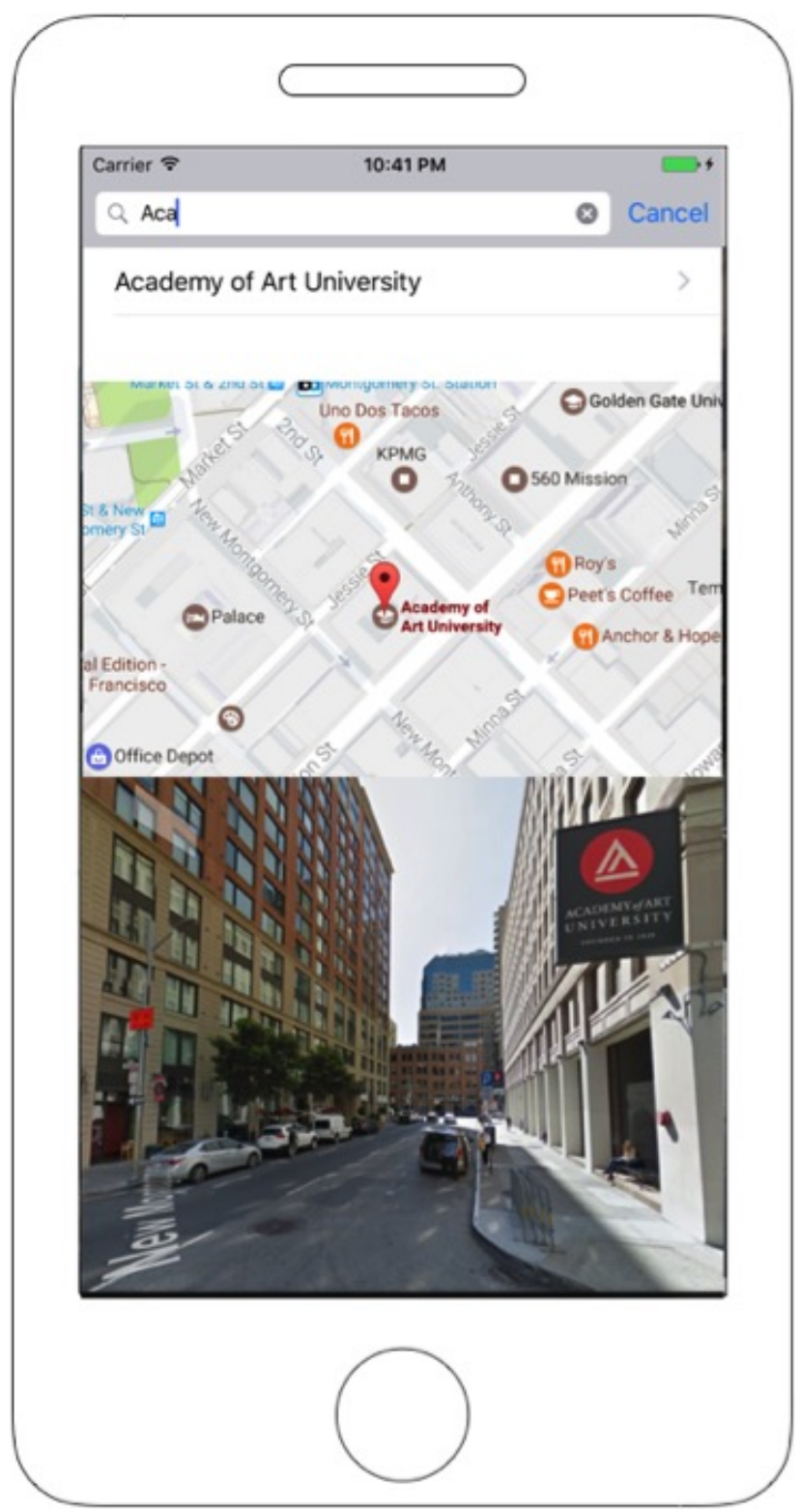

Figure 9.12: A suggested encoding interface combing Street View and Map View for LBR designs 
locations through one search bar. For example, "to get gas" should allow users to search a particular business like "Shell" or a category of locations such as gas station.

\subsection{Summary}

Future LBR designers might want to consider using Street View at the LBR encoding stage. The results of this evaluation study provided some possible design implications about how to apply the Street View into the encoding interface for a LBR: 1) Combing Street View with Map View at the encoding interface to make sure people learn spatial relations about the target location 2) Providing different location information for familiar places and unfamiliar places. 


\section{Chapter 10}

\section{Conclusions and Future Work}

\subsection{Conclusions}

In this dissertation, I have applied human prospective memory theory into the technical design of LBRs to make it work more consistently with how human memory works. The goal is to potentially avoid attenuating human's remembering ability.

I first investigated the existing research challenges in the domain of LBRs. I conducted a usability evaluation study on an existing commercial LBR. The study results showed that current design of LBRs doesn't support effective reminder delivery. Current design fails to address how human remember and recognize location information using their internal memory. As a follow up, I set up an online survey to understand how people remember and organize location-based to-do tasks in their daily lives. According to the data collected from the survey, I proposed a classification of different uses of locations for remembering location-based to-do tasks in people's real life.

By understanding how people use their prospective memory to remember future to-do tasks, 
I proposed a framework connecting prospective memory with LBRs. Research problems were identified in this framework: 1) the encoding interface of existing designs does not match the view people encounter at the retrieval stage, 2) the prior familiarity with the place is not applied in the current LBR design to support location recognition at the retrieval stage. I came up two main research questions regarding to these problems. My dissertation is based on this theoretical framework.

Then I conducted two experiments to address the two main research questions in this dissertation. In the first experiment, I focused on studying the match between the encoding stage and the retrieval stage. The results demonstrated that providing a street view an the encoding stage benefits prospective remembering. The second experiment studied the impact of familiarity of business location and the city on recognizing the location at a later time. The results suggested that future LBR designers should consider user's familiarity level with the places and adapt the encoding interface appropriately.

Finally, I designed a LBR prototype by applying the research findings from these user studies. Street View was applied at the encoding interface to see how users actually understand and use it to set up a location-based reminder. Furthermore, I provided two types of locations at the encoding interface. The goal was to see how users perceive the differences between the two types of locations and how they use them to set up reminders.

\subsection{Future Work}

My doctoral dissertation extended LBRs to a new form of external memory aid by applying basic psychological research findings into the technical design of LBRs. My research opens some possible areas of future work. 


\subsubsection{Route Planner With LBR}

As mentioned by several participants, LBRs can be more useful if it could deliver the reminder and provide route information at the beginning of a trip. In real use cases, it is too late to trigger the reminder when the user is already near the location. It makes more sense to the user that a list of reminders pops up indicating the possible to-do tasks along the route when the user is leaving a place. The goal is to support people in getting things done more efficiently while they are on the way. Future work should aim to design more adaptive and efficient LBRs by understanding users' needs of using LBRs in their real life.

\subsubsection{Context-aware Reminder}

Context-awareness is important in determining the type and nature of human activity while people are using mobile devices. Today, mobile services are commonly used in people's daily lives as their information needs vary depending on their moment-to-moment location. Depending on where, when, how, with whom, and why customers are navigating in physical space, their needs vary. Humans are really good at knowing contexts, but technology is not. I would like to expand my research to future context-aware mobile application design: that is mobile devices should be able to capture and process the changing locations and contexts, and provide adaptive user interfaces.

In this dissertation, I found that participants have difficulties using external reminders effectively: sometimes they bring the coupon while shopping grocery, but forget to use it when checking out. This is a typical scenario where reminders are set up, but they are not triggered effectively. In this case, anything in the environment can possibly serve as an external cue to trigger the use of the coupon. For example, the trigger can be the person in front of the checkout counter, it can be the coupon itself on the desk, it also can be a scenario of someone 
else using a coupon. Ubiquitous technology today allows people to record many life moments and use them. In my future work, I would like to automatically provide relevant contextual cues from the environment by utilizing existing ubiquitous infrastructure. I would like to understand what information in the environment can be used as external cues to trigger human's memory to remember the task. To be more specific, how there external cues can be used effectively to trigger human's remembering of a to-do task at that particular moment. My ultimate goal aims to complement and enhance human memory with technology, rather than replacing human memory with technology.

\subsubsection{What Information to Remind}

This dissertation focused a lot on supporting location recognition, very few research was conducted to support task recall. In some cases, people know exactly what they need to do without checking the reminder when a reminder is delivered at a particular time. In other times, people remember that they need to something, but they forget what to do. The associative link between the external cue and the intended action needs to be further studied. I am interested in exploring external cues to support task recall at the retrieval stage. For example, what task information should be provided at the encoding stage to benefit task recall at the retrieval stage. 


\section{Bibliography}

[Abowd et al., 1999] Abowd, G. D., Dey, A. K., Brown, P. J., Davies, N., Smith, M., and Steggles, P. (1999). Towards a better understanding of context and context-awareness. In Handheld and ubiquitous computing, pages 304-307. Springer.

[Acredolo, 1982] Acredolo, L. P. (1982). The familiarity factor in spatial research. New Directions for Child and Adolescent Development, 1982(15):19-30.

[Beigl, 2000] Beigl, M. (2000). Memoclip: A location-based remembrance appliance. Personal and Ubiquitous Computing, 4(4):230-233.

[Brown, 1995] Brown, P. J. (1995). The stick-e document: a framework for creating contextaware applications. ELECTRONIC PUBLISHING-CHICHESTER-, 8:259-272.

[Burrell and Gay, 2002] Burrell, J. and Gay, G. K. (2002). E-graffiti: evaluating real-world use of a context-aware system. Interacting with Computers, 14(4):301-312.

[Cakmak and Basoglu, 2012] Cakmak, N. and Basoglu, N. (2012). An investigation of factors that influence user intention to use location based mobile applications. In Technology Management for Emerging Technologies (PICMET), 2012 Proceedings of PICMET'12:, pages 276-285. IEEE. 
[Church and de Oliveira, 2013] Church, K. and de Oliveira, R. (2013). What's up with whatsapp?: comparing mobile instant messaging behaviors with traditional sms. In Proceedings of the 15th international conference on Human-computer interaction with mobile devices and services, pages 352-361. ACM.

[Cockburn, 1996] Cockburn, J. (1996). Assessment and treatment of prospective memory deficits. Prospective memory: Theory and applications, pages 327-350.

[Darken and Sibert, 1996] Darken, R. P. and Sibert, J. L. (1996). Wayfinding strategies and behaviors in large virtual worlds. In Proceedings of the SIGCHI conference on Human factors in computing systems, pages 142-149. ACM.

[DeVaul et al., 2003] DeVaul, R. W., Corey, V. R., et al. (2003). The memory glasses: subliminal vs. overt memory support with imperfect information. In 2012 16th International Symposium on Wearable Computers, pages 146-146. IEEE Computer Society.

[Dey and Abowd, 2000] Dey, A. K. and Abowd, G. D. (2000). Cybreminder: A contextaware system for supporting reminders. In Handheld and Ubiquitous Computing, pages 172-186. Springer.

[Dismukes, 2010] Dismukes, R. K. (2010). Remembrance of things future: prospective memory in laboratory, workplace, and everyday settings. Reviews of human factors and ergonomics, 6(1):79-122.

[Einstein and McDaniel, 1990] Einstein, G. O. and McDaniel, M. A. (1990). Normal aging and prospective memory. Journal of Experimental Psychology: Learning, Memory, and Cognition, 16(4):717. 
[Einstein and McDaniel, 2005] Einstein, G. O. and McDaniel, M. A. (2005). Prospective memory multiple retrieval processes. Current Directions in Psychological Science, $14(6): 286-290$.

[Einstein et al., 1996] Einstein, G. O., McDaniel, M. A., Brandimonte, M., Einstein, G., and McDaniel, M. (1996). Retrieval processes in prospective memory: Theoretical approaches and some new empirical findings. Prospective memory: Theory and applications, pages $115-141$.

[Ellis, 1996] Ellis, J. (1996). Prospective memory or the realization of delayed intentions: A conceptual framework for research. Prospective memory: Theory and applications, pages $1-22$.

[Elsweiler et al., 2007] Elsweiler, D., Ruthven, I., and Jones, C. (2007). Towards memory supporting personal information management tools. Journal of the American Society for Information Science and Technology, 58(7):924-946.

[Elsweiler et al., 2006] Elsweiler, D., Ruthven, I., and Ma, L. (2006). Considering human memory in pim. In SIGIR 2006 Workshop on Personal Information Management, August, pages 10-11. Citeseer.

[Espinoza et al., 2001] Espinoza, F., Persson, P., Sandin, A., Nyström, H., Cacciatore, E., and Bylund, M. (2001). Geonotes: Social and navigational aspects of location-based information systems. In Ubicomp 2001: Ubiquitous Computing, pages 2-17. Springer.

[Frank et al., 2004] Frank, C., Caduff, D., and Wuersch, M. (2004). From gis to lbs-an intelligent mobile gis. IfGI prints, 22:261-274. 
[Gärling et al., 1983] Gärling, T., Lindberg, E., and Mäntylä, T. (1983). Orientation in buildings: effects of familiarity, visual access, and orientation aids. Journal of Applied Psychology, 68(1):177.

[Golledge, 1992] Golledge, R. G. (1992). Place recognition and wayfinding: Making sense of space. Geoforum, 23(2):199-214.

[Gollwitzer, 1999] Gollwitzer, P. M. (1999). Implementation intentions: strong effects of simple plans. American psychologist, 54(7):493.

[Graf and Uttl, 2001] Graf, P. and Uttl, B. (2001). Prospective memory: A new focus for research. Consciousness and Cognition, 10(4):437-450.

[Griswold et al., 2004] Griswold, W. G., Shanahan, P., Brown, S. W., Boyer, R., Ratto, M., Shapiro, R. B., and Truong, T. M. (2004). Activecampus: experiments in communityoriented ubiquitous computing. Computer, 37(10):73-81.

[Guynn et al., 1998] Guynn, M. J., Mcdaniel, M. A., and Einstein, G. O. (1998). Prospective memory: When reminders fail. Memory $\&$ Cognition, 26(2):287-298.

[Gwizdka, 2001] Gwizdka, J. (2001). Supporting prospective information in email. In CHI'01 Extended Abstracts on Human Factors in Computing Systems, pages 135-136. ACM.

[Harris, 1978] Harris, J. E. (1978). External memory aids. Practical aspects of memory, 1:172.

[Harris, 1980] Harris, J. E. (1980). Memory aids people use: Two interview studies. Memory E Cognition, 8(1):31-38.

[Herrmann and Petro, 1990] Herrmann, D. J. and Petro, S. J. (1990). Commercial memory aids. Applied Cognitive Psychology, 4(6):439-450. 
[Hirtle, 2011] Hirtle, S. C. (2011). Geographical design: Spatial cognition and geographical information science. Synthesis Lectures on Human-Centered Informatics, 4(1):1-67.

[Hirtle and Frank, 1997] Hirtle, S. C. and Frank, A. U. (1997). Spatial Information Theory A Theoretical Basis for GIS: International Conference COSIT'97, Laurel Highlands, Pennsylvania, USA, October 15-18, 199\%. Proceedings, volume 1329. Springer Science \& Business Media.

[Hirtle and Raubal, 2013] Hirtle, S. C. and Raubal, M. (2013). Many to many mobile maps. In Cognitive and Linguistic Aspects of Geographic Space, pages 141-157. Springer.

[Hirtle and Sorrows, 1998] Hirtle, S. C. and Sorrows, M. E. (1998). Designing a multi-modal tool for locating buildings on a college campus. Journal of Environmental Psychology, $18(3): 265-276$.

[Intons-Peterson and Fournier, 1986] Intons-Peterson, M. J. and Fournier, J. (1986). External and internal memory aids: When and how often do we use them? Journal of Experimental Psychology: General, 115(3):267.

[Intons-Peterson and Newsome III, 1992] Intons-Peterson, M. J. and Newsome III, G. L. (1992). External memory aids: Effects and effectiveness. In Memory Improvement, pages 101-121. Springer.

[Jiang and Yao, 2006] Jiang, B. and Yao, X. (2006). Location-based services and gis in perspective. Computers, Environment and Urban Systems, 30(6):712-725.

[Kaasinen, 2003] Kaasinen, E. (2003). User needs for location-aware mobile services. Personal and ubiquitous computing, 7(1):70-79. 
[Kwon et al., 2005] Kwon, O., Choi, S., and Park, G. (2005). Nama: A context-aware multi-agent based web service approach to proactive need identification for personalized reminder systems. Expert Systems with Applications, 29(1):17-32.

[Lamming and Flynn, 1994] Lamming, M. and Flynn, M. (1994). Forget-me-not: Intimate computing in support of human memory. In Proc. FRIEND21, 1994 Int. Symp. on Next Generation Human Interface, page 4. Citeseer.

[Li et al., 2005] Li, K. A., Sohn, T., and Griswold, W. G. (2005). Evaluating Location Based Reminders. Department of Computer Science and Engineering, University of California, San Diego.

[Lin et al., 2012] Lin, C.-Y., Hung, M.-T., and Huang, W.-H. (2012). A location-based personal task management application for indoor and outdoor environments. In NetworkBased Information Systems (NBiS), 2012 15th International Conference on, pages 582587. IEEE.

[Liu et al., 2011] Liu, S., Li, Y., Guo, A., and Lim, J. H. (2011). Advertisement image recognition for a location-based reminder system. In Advances in Multimedia Modeling, pages 421-431. Springer.

[Ludford et al., 2006] Ludford, P. J., Frankowski, D., Reily, K., Wilms, K., and Terveen, L. (2006). Because i carry my cell phone anyway: functional location-based reminder applications. In Proceedings of the SIGCHI conference on Human Factors in computing systems, pages 889-898. ACM.

[Marmasse and Schmandt, 2000] Marmasse, N. and Schmandt, C. (2000). Location-aware information delivery withcommotion. In Handheld and Ubiquitous Computing, pages 157171. Springer. 
[McDaniel and Einstein, 1993] McDaniel, M. A. and Einstein, G. O. (1993). The importance of cue familiarity and cue distinctiveness in prospective memory. Memory, 1(1):23-41.

[McDaniel and Einstein, 2000] McDaniel, M. A. and Einstein, G. O. (2000). Strategic and automatic processes in prospective memory retrieval: A multiprocess framework. Applied cognitive psychology, 14(7):S127-S144.

[McDaniel et al., 2004] McDaniel, M. A., Guynn, M. J., Einstein, G. O., and Breneiser, J. (2004). Cue-focused and reflexive-associative processes in prospective memory retrieval. Journal of Experimental Psychology: Learning, Memory, and Cognition, 30(3):605.

[McDonald et al., 2011] McDonald, A., Haslam, C., Yates, P., Gurr, B., Leeder, G., and Sayers, A. (2011). Google calendar: A new memory aid to compensate for prospective memory deficits following acquired brain injury. Neuropsychological rehabilitation, 21(6):784-807.

[Morita, 2006] Morita, T. (2006). Reminders supporting spontaneous remembering in prospective memory tasks1. Japanese Psychological Research, 48(1):34-39.

[Morrison et al., 2004] Morrison, K., Szymkowiak, A., and Gregor, P. (2004). Memojogan interactive memory aid incorporating mobile based technologies. In Mobile HumanComputer Interaction-MobileHCI 2004, pages 481-485. Springer.

[Nielsen, 2005] Nielsen, J. (2005). Ten usability heuristics. Retrieved January 5th, 2015, from Nielsen Norman Group.

[Ockerman and Pritchett, 2000] Ockerman, J. and Pritchett, A. (2000). A review and reappraisal of task guidance: Aiding workers in procedure following. International Journal of Cognitive Ergonomics, 4(3):191-212.

[O’Neill, 1992] O'Neill, M. J. (1992). Effects of familiarity and plan complexity on wayfinding in simulated buildings. Journal of Environmental Psychology, 12(4):319-327. 
[Payne, 1993] Payne, S. J. (1993). Understanding calendar use. Human-Computer Interaction, 8(2):83-100.

[Rao and Minakakis, 2003] Rao, B. and Minakakis, L. (2003). Evolution of mobile locationbased services. Communications of the ACM, 46(12):61-65.

[Sahami Shirazi et al., 2014] Sahami Shirazi, A., Henze, N., Dingler, T., Pielot, M., Weber, D., and Schmidt, A. (2014). Large-scale assessment of mobile notifications. In Proceedings of the SIGCHI Conference on Human Factors in Computing Systems, pages 3055-3064. ACM.

[Schacter, 1999] Schacter, D. L. (1999). The seven sins of memory: Insights from psychology and cognitive neuroscience. American psychologist, 54(3):182.

[Sohn et al., 2005] Sohn, T., Li, K. A., Lee, G., Smith, I., Scott, J., and Griswold, W. G. (2005). Place-its: A study of location-based reminders on mobile phones. Springer.

[Sparrow et al., 2011] Sparrow, B., Liu, J., and Wegner, D. M. (2011). Google effects on memory: Cognitive consequences of having information at our fingertips. science, $333(6043): 776-778$.

[Stevens and Coupe, 1978] Stevens, A. and Coupe, P. (1978). Distortions in judged spatial relations. Cognitive psychology, 10(4):422-437.

[Storm et al., 2016] Storm, B. C., Stone, S. M., and Benjamin, A. S. (2016). Using the internet to access information inflates future use of the internet to access other information. Memory, pages 1-7.

[Tamminen et al., 2004] Tamminen, S., Oulasvirta, A., Toiskallio, K., and Kankainen, A. (2004). Understanding mobile contexts. Personal and ubiquitous computing, 8(2):135143. 
[Thöne-Otto and Walther, 2003] Thöne-Otto, A. I. and Walther, K. (2003). How to design an electronic memory aid for brain-injured patients: Considerations on the basis of a model of prospective memory. International Journal of Psychology, 38(4):236-244.

[Thorndyke and Goldin, 1983] Thorndyke, P. W. and Goldin, S. E. (1983). Spatial learning and reasoning skill. In Spatial orientation, pages 195-217. Springer.

[Thorndyke and Hayes-Roth, 1982] Thorndyke, P. W. and Hayes-Roth, B. (1982). Differences in spatial knowledge acquired from maps and navigation. Cognitive psychology, $14(4): 560-589$.

[Thorndyke et al., 1980] Thorndyke, P. W., Hayes-Roth, B., and Stasz, C. (1980). Performance models for spatial and locational cognition. Rand.

[Tu et al., 2013] Tu, Y., Chen, L., Lv, M., Ye, Y., Huang, W., and Chen, G. (2013). ireminder: An intuitive location-based reminder that knows where you are going. International Journal of Human-Computer Interaction, 29(12):838-850.

[Tversky, 1993] Tversky, B. (1993). Cognitive maps, cognitive collages, and spatial mental models. In European Conference on Spatial Information Theory, pages 14-24. Springer.

[van den Hoven and Eggen, 2014] van den Hoven, E. and Eggen, B. (2014). The cue is key: Design for real-life remembering. Zeitschrift für Psychologie, 222(2):110.

[Vemuri and Bender, 2004] Vemuri, S. and Bender, W. (2004). Next-generation personal memory aids. BT Technology Journal, 22(4):125-138.

[Vortac et al., 1995] Vortac, O., Edwards, M. B., and Manning, C. A. (1995). Functions of external cues in prospective memory. Memory, 3(2):201-219. 
[Wang and Loui, 2009] Wang, J. L. and Loui, M. C. (2009). Privacy and ethical issues in location-based tracking systems. In Technology and Society, 2009. ISTAS'09. IEEE International Symposium on, pages 1-4. IEEE.

[Wang and Perez-Quinones, 2015] Wang, Y. and Perez-Quinones, M. A. (2015). Beyond geofencing: Specifying location in location-based reminder applications. In Proceedings of the 33rd Annual ACM Conference Extended Abstracts on Human Factors in Computing Systems, pages 1767-1772. ACM.

[Wickens, 1992] Wickens, C. D. (1992). Engineering psychology and human performance . HarperCollins Publishers.

[Zhou et al., 2012] Zhou, S., Chu, C.-H., Yu, Z., and Kim, J. (2012). A context-aware reminder system for elders based on fuzzy linguistic approach. Expert Systems with Applications, 39(10):9411-9419. 
Appendix A

Survey Materials for Chapter 5 


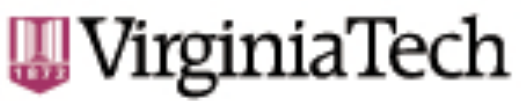

Office of Research Compliance

Institutional Review Board

North End Center, Suite 4120, Virginia Tech

300 Turner Street NW

Blacksburg, Virginia 24061

540/231-4606 Fax 540/231-0959

email irb@vt.edu

website http://www.irb.vt.edu

MEMORANDUM

DATE:

TO:

FROM:
October 10, 2014

Manuel A Perez-Quinonez, Yao Wang

Virginia Tech Institutional Review Board (FWA00000572, expires April 25, 2018)

\section{IRB NUMBER:}

Collect daily to-do tasks

Effective October 10, 2014, the Virginia Tech Institution Review Board (IRB) Chair, David M Moore, approved the New Application request for the above-mentioned research protocol.

This approval provides permission to begin the human subject activities outlined in the IRB-approved protocol and supporting documents.

Plans to deviate from the approved protocol and/or supporting documents must be submitted to the IRB as an amendment request and approved by the IRB prior to the implementation of any changes, regardless of how minor, except where necessary to eliminate apparent immediate hazards to the subjects. Report within 5 business days to the IRB any injuries or other unanticipated or adverse events involving risks or harms to human research subjects or others.

All investigators (listed above) are required to comply with the researcher requirements outlined at:

http://www.irb.vt.edu/pages/responsibilities.htm

(Please review responsibilities before the commencement of your research.)

\section{PROTOCOL INFORMATION:}

Approved As:

Protocol Approval Date:

Protocol Expiration Date:

\section{Exempt, under 45 CFR 46.110 category(ies) 2}

October 10, 2014

N/A

N/A

*Date a Continuing Review application is due to the IRB office if human subject activities covered under this protocol, including data analysis, are to continue beyond the Protocol Expiration Date.

\section{FEDERALLY FUNDED RESEARCH REQUIREMENTS:}

Per federal regulations, 45 CFR 46.103(f), the IRB is required to compare all federally funded grant proposals/work statements to the IRB protocol(s) which cover the human research activities included in the proposal / work statement before funds are released. Note that this requirement does not apply to Exempt and Interim IRB protocols, or grants for which VT is not the primary awardee.

The table on the following page indicates whether grant proposals are related to this IRB protocol, and which of the listed proposals, if any, have been compared to this IRB protocol, if required. 


\begin{tabular}{|l|l|l|l|}
\hline Date* & OSP Number & Sponsor & Grant Comparison Conducted? \\
\hline & & & \\
\hline & & & \\
\hline & & & \\
\hline & & & \\
\hline & & & \\
\hline & & & \\
\hline & & & \\
\hline & & & \\
\hline
\end{tabular}

* Date this proposal number was compared, assessed as not requiring comparison, or comparison information was revised.

If this IRB protocol is to cover any other grant proposals, please contact the IRB office (irbadmin@vt.edu) immediately. 
You must be 18 years of age or older to be eligible to participate in this study. Please select the appropriate option below.

a. I certify that I am 18 years old or older.

b. I am younger than 18 years old.

We are studying how people remember and manage things to-do in the future. For example, how do you remember to buy a ticket for an upcoming event? Do you rely on your memory or a to-do list? For this survey, we are requesting you to provide 35 real to-do tasks that you intend to do in the future. It can be any to-do tasks related with your work, your life or anything else.

\section{Tasks}

To-do task 1

2. How frequently do you usually execute this task?
a. Very Frequently
b. Frequently
c. Sometimes
d. Occasionally
e. Rarely

3. How important do you consider this task to be?
a. Essential
b. Very important
c. Somewhat important
d. A little important
e. Not important

4. How frequently do you forget to do this task?
a. Very Frequently
b. Frequently
c. Sometimes
d. Occasionally
e. Rarely

5. How difficult of remembering this to-do task in the memory?
a. Very difficult
b. Difficult
c. Neutral
d. Easy
e. Very easy

Done/ Add one more 


\section{Post Questions}

1. Do you use any devices or techniques to support your memory?

a) Yes

b) No

If Yes is selected, which of the following do you make use of? You can choose more than one.

- Computer-based

$\circ$ Alarm clocks and timers

- Web bookmarks

○ Send self text messages

- Email folders, use inbox to remember a to-do task

- Calendar entries

- Notes/To-dos on the phone

- Reminder tools on the phone

o Others

- Paper-based

○ Shopping lists

- To-do lists

- Post-it notes

- Address Books

○ Dairy/Journal

- Others

2. When you place an item in a to-do list, do you usually specify the location for each task (do you consider where to finish the task)?

a) Yes

b) No

3. Simply explain your behaviors of how you organize your to-do tasks to make sure you remember to do it at a later time.

Thank you for your response. Please answer the following demographic questions and click the submit button to finish the survey. We appreciate your help.

1. How old are you?

2. What is your current level of education?
a) Undergraduate
b) Graduate student
c) Faculty member
d) Industry employee
e) Other

If industry employee is selected, do not display question 3

3. What is your department? 
Appendix B

User Study Materials for Chapter 7 


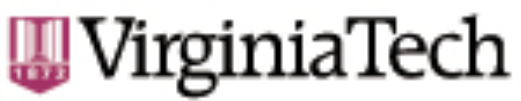

Office of Research Compliance

Institutional Review Board

North End Center, Suite 4120, Virginia Tech

300 Turner Street NW

Blacksburg, Virginia 24061

540/231-4606 Fax 540/231-0959

email irb@vt.edu

website http://www.irb.vt.edu

MEMORANDUM

DATE:

TO:

FROM:
December 11, 2014

Manuel A Perez-Quinonez, Yao Wang

Virginia Tech Institutional Review Board (FWA00000572, expires April 25, 2018)

Exploring the effectiveness of external memory cues

14-918

\section{IRB NUMBER:}

Effective December 10, 2014, the Virginia Tech Institution Review Board (IRB) Chair, David M Moore, approved the New Application request for the above-mentioned research protocol.

This approval provides permission to begin the human subject activities outlined in the IRB-approved protocol and supporting documents.

Plans to deviate from the approved protocol and/or supporting documents must be submitted to the IRB as an amendment request and approved by the IRB prior to the implementation of any changes, regardless of how minor, except where necessary to eliminate apparent immediate hazards to the subjects. Report within 5 business days to the IRB any injuries or other unanticipated or adverse events involving risks or harms to human research subjects or others.

All investigators (listed above) are required to comply with the researcher requirements outlined at:

http://www.irb.vt.edu/pages/responsibilities.htm

(Please review responsibilities before the commencement of your research.)

\section{PROTOCOL INFORMATION:}

Approved As:

Protocol Approval Date:

Protocol Expiration Date:
Expedited, under 45 CFR 46.110 category(ies) 6,7

December 10, 2014

December 9, 2015

Continuing Review Due Date*: November 25, 2015

*Date a Continuing Review application is due to the IRB office if human subject activities covered under this protocol, including data analysis, are to continue beyond the Protocol Expiration Date.

\section{FEDERALLY FUNDED RESEARCH REQUIREMENTS:}

Per federal regulations, 45 CFR 46.103(f), the IRB is required to compare all federally funded grant proposals/work statements to the IRB protocol(s) which cover the human research activities included in the proposal / work statement before funds are released. Note that this requirement does not apply to Exempt and Interim IRB protocols, or grants for which VT is not the primary awardee.

The table on the following page indicates whether grant proposals are related to this IRB protocol, and which of the listed proposals, if any, have been compared to this IRB protocol, if required. 


\begin{tabular}{|l|l|l|l|}
\hline Date* & OSP Number & Sponsor & Grant Comparison Conducted? \\
\hline & & & \\
\hline & & & \\
\hline & & & \\
\hline & & & \\
\hline & & & \\
\hline & & & \\
\hline & & & \\
\hline & & & \\
\hline
\end{tabular}

* Date this proposal number was compared, assessed as not requiring comparison, or comparison information was revised.

If this IRB protocol is to cover any other grant proposals, please contact the IRB office (irbadmin@vt.edu) immediately. 


\section{Virginia Polytechnic and State University Center for Human-Computer Interaction $(\mathrm{CHCl})$ CONSENT FORM}

You are hereby invited to participate in a research study. Please read through the information below carefully and ask any questions that you may have before agreeing to take part in the study.

What the Study is About: The purpose of the study is to understand how people remember and manage their daily to-do tasks using reminders. We are trying to explore the human behaviors of encoding and remembering future to-do tasks.

What You will be Asked to Do If you agree to participate in this study, you will be engaged in a two-part of the study, each part will take no more than one hour in the $\mathrm{HCl}$ lab on campus in MCB room 133. The first part of the study will ask you to remember some predefined to-do tasks that you need to finish in the future, and the second part of the study will ask you to come back to see how many tasks you can recall.

Risks and Benefits: The results of this study will contribute to academic knowledge, and to understanding how people recall to-do tasks and how they use reminders to assist daily remembering. It is not intended to have any direct benefits or risks to you as a participant. Participants will receive SONA credits if they use SONA system to register the study.

Data Collected will be Confidential: The records of this study will be kept private. Your name will not be attached in any way to your responses or to your participation in the study. Research data will be kept in a secure computer, and only the researchers will have access to the records. The data will only be used for publication purposes.

Audio Recording: With your permission, we will capture the computer screen and audio record your comments. No private information such as name will be stored together with the recordings to identify you.

Taking Part is Voluntary: Your participation in this study is completely voluntary. You may discontinue participation at any time prior to the completion of any part of our study.

If You have any Questions or Inquiries: You may contact Yao Wang at yxw5167@vt.edu or Dr. Manuel A Perez-Quinones at perez@vt.edu for questions about the study and Dr. David Moore at moored@vt.edu or 231-4991 for questions related to your rights as a human subject research participant. 


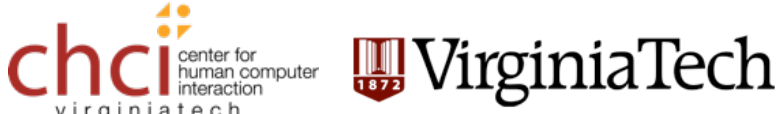

\section{CONSENT}

You must be 18 years older to be eligible to participate in this study. I certify that I am 18 years old or older. I have read and understand the above information. I have received a copy of this form. I agree to participate in this study.

Date 


\section{Background Questions, give paper to the participants before the study}

1. What is your age?

2. What is your gender?

3. How would you describe your race?
a. White, Caucasian
b. Black, African American
c. American Indian or Alaska Native
d. Asian
e. Native Hawaiian or other Pacific Islander
f. Some other race or origin
g. Prefer not to disclose

4. Are you of Hispanic/Latino/Spanish origin?
a. Yes
b. No
c. Prefer not to disclose

5. What is your dominant language (sometimes known as first language)?

6. What degree are you pursing (bachelors, masters, doctorate?

7. What major are you pursuing?

8. How familiar are you with the San Francisco downtown area?

a. Have never been there before

b. Have traveled there, then how many times

c. Lived there for a couple of months, then how many months

d. Have lived there for a couple of years, then how many years

e. Others

On a scale of 1-5 to rate how often do the following situations occur to you.

9. Forget an appointment

1

Never
2

Rarely
3

Sometimes
4

Most of the time
5

Always

10. Forget to pay a bill 
1

Never
2

Rarely
3

Sometimes
4

Most of the time
5

Always

11. Forget to pick up or buy something at a particular place

$\begin{array}{lllll}1 & 2 & 3 & 4 & 5 \\ \text { Never } & \text { Rarely } & \text { Sometimes } & \begin{array}{l}\text { Most of the } \\ \text { time }\end{array} & \text { Always }\end{array}$

12. Forget to bring something with you

1

Never
2

Rarely
3

Sometimes
4

Most of the time
5

Always

13. Forget to return something or drop something at a particular place

1

Never
2

Rarely
3

Sometimes
4

Most of the time
5

Always 


\section{Moderator Protocol}

Thank you for your time today. This study aims to help us understand how people remember their to-do tasks.

First of all, I need to make sure you are older than 18 and then sign the consent form.

[Give the consent form]

Now I am going to assign you a participant ID, which is used during the study to fill out the survey questions. Your participant ID is xxx.

\section{Session 1}

This study consists of two sessions, and each session takes no more than 30 minutes. You will need to come back after 3 days to complete the second session.

In the first session of the study, I will present eight location-based tasks you need to remember to do at a later time. In the second session of the study, I will ask you to recall the tasks and locations that you memorized during the first session. You will have to recall the location of where the task needs to done and the task of what needs to be done.

For both sessions, I will screen capture the computer and audio record your comments. The recordings will be only used for research purposes.

Ready to start?

[Start recording]

We will move forward to the first session now. During the study, feel free to take a rest at any time. Thanks again for your time.

First please complete some background questions.

[Open background questions on Qualtrics]

Now, I am going to give you a list of eight pre-defined to-do tasks, you will enter each task into the computer as if you were adding it to your to-do list; you can type in whatever you would normally do on your real to-do list. At the same time, you will see images representing the business locations you need to visit to complete that task.

Note $^{1}$ : content in the [] is used as a reminder for the moderator to conduct the study. 
Once you have finished entering one task, just click the "next task" button on the computer to move to the next task. After you have finished entering all the eight tasks, click the "I am done" button.

[Give the task scenarios and open the web page for users]

You have finished entering all the to-do tasks. You will have to remember these tasks when you come back in a few days. I would like to confirm the time and date for session 2 .

This is all for the first session of the study. Thank you very much for your time today. I will see you in a couple of days.

\section{Session 2}

Welcome back. Again, thank you very much for your time. In this session, I will ask you to recall the to-do tasks that you entered into the computer several days ago.

On the screen, you will see a video taken from a car driving through downtown San Francisco. I would like you to imagine that you are driving the car. As you drive, I want you to count how many traffic lights you come across. Just count the ones you are passing by. I will give you a piece of paper and a pen, so you can use them to count the traffic lights if you want. At the same time, when you see one of the business locations you need to visit, please pause the video and say out loud the task you need to complete at that location.

[Give pen and paper]

First, you can complete a practice session to make sure you understand the task. Image that you need to get a sandwich from Subway. While you are watching the video, you need to count the traffic lights you are passing by and remember to pause the video at where you need to finish this task.

[Play 20 seconds exercise video]

As a reminder, I will screen capture the computer and audio record your comments. The recordings will be only used for research purposes.

[Start recording]

You have a total of eight tasks to identify while you are watching the video. This part of the study is completed when the video comes to the end.

Before we start, do you need some time to think about the to-do tasks?

Note $^{1}$ : content in the [] is used as a reminder for the moderator to conduct the study. 
[Play the video]

Now, I would like you to complete the following questions.

Please rate the following questions to assess your effort doing the task you have just completed.

[Open TLX questions on the Qualtrics]

Please rate the following questions based on your general experiences in daily life. [Open location and task questions on the Qualtrics]

This is the end of the study. Thank you very much for your interests. If you have any further questions and concerns regarding the study, please send me an email at any time.

Note $^{1}$ : content in the [] is used as a reminder for the moderator to conduct the study. 


\section{Session 1 To-do tasks}

Imagine it is $10 \mathrm{am}$ on Friday; you are at work now. You want to clean up the tasks that you need to complete later, so you start to prepare a to-do list with the tasks listed below. You need to enter all these to-do tasks into the computer.

1. You ordered a wireless printer online, but shipping is expensive. You decided to ship it to the Office Depot for free. It has arrived, and you want to remember to pick up the printer while you are driving by the Office Depot in downtown.

Once you have finished remembering this task, you can click the "next task" button to continue.

2. Your friend Tom borrowed a book from the library at the Academy of Art University. He is out of town now and the due date of book is today. AAU is near your workplace, so he asked you to return the book for him. You need to remember to return the book this afternoon.

Once you have finished remembering this task, you can click the "next task" button to continue.

3. Your roommate helped you solve a big problem in your homework yesterday. Peets Coffee and Tea was offering limited holiday specials. As a thank you, you would like to stop by Peets Coffee and Tea to buy a holiday gift for your roommate.

Once you have finished remembering this task, you can click the "next task" button to continue.

4. You live in an apartment, and you need some quarters to use the washer and dryer in the basement. There is a Wells Fargo bank near the workplace, so you want to get some quarters at that Wells Fargo.

Once you have finished remembering this task, you can click the "next task" button to continue.

5. The fall season is coming. You are having seasonal allergy. You would like to stop by Walgreens to pick up the allergies prescription drugs.

Once you have finished remembering this task, you can click the "next task" button to continue. 
6. Your mother's birthday is coming. You would like to mail a package to your Mom as a gift. You need to go to FedEx to drop the package.

Once you have finished remembering this task, you can click the "next task" button to continue.

7. You received a promotional letter from Citibank, so you decided to open a new credit card at Citibank this afternoon. You need to apply in person before the promotion expires today.

Once you have finished remembering this task, you can click the "next task" button to continue.

8. You are going to host a birthday party in your house this weekend. Target is on your way home; so you decided to stop at Target to buy groceries.

Once you have finished remembering this task, you can click the "I am done" button to complete the study. 
Please rate the following questions based on your general experiences in daily life.

Before you completed this experiment, on a scale of 1-5 to rate how familiar are you with the businesses listed below and their logos.

at all
Not familiar

Very familiar

Office Depot

1

2

3

4

5

Academy of Art

1

2

3

4

5

University

Peets Coffee and

1

2

3

4

5

Tea

Wells Fargo Bank

1

2

3

4

5

Walgreens

1

2

3

4

5

Fedex

Citibank

Target
2

2

2
3

3

3
4

5

5

5 
On a scale of 1-5 to rate how frequently do you perform this task in your real daily life.

Very
infrequently

\begin{abstract}
Order something
online and pick up

in store
\end{abstract}

Return a book to the library

\begin{tabular}{|c|c|c|c|c|c|}
\hline $\begin{array}{c}\text { Return a book to the } \\
\text { library }\end{array}$ & 1 & 2 & 3 & 4 & 5 \\
\hline $\begin{array}{l}\text { Buy gifts at a coffee } \\
\text { shop }\end{array}$ & 1 & 2 & 3 & 4 & 5 \\
\hline $\begin{array}{l}\text { Get change (e.g., } \\
\text { quarters) }\end{array}$ & 1 & 2 & 3 & 4 & 5 \\
\hline $\begin{array}{l}\text { Pick up prescription } \\
\text { drugs }\end{array}$ & 1 & 2 & 3 & 4 & 5 \\
\hline Mail a package & 1 & 2 & 3 & 4 & 5 \\
\hline $\begin{array}{l}\text { Open a new credit } \\
\text { card }\end{array}$ & 1 & 2 & 3 & 4 & 5 \\
\hline $\begin{array}{l}\text { Do grocery } \\
\text { shopping }\end{array}$ & 1 & 2 & 3 & 4 & 5 \\
\hline
\end{tabular}

Very

frequently 
Appendix C

\section{User Study Materials for Chapter 8}




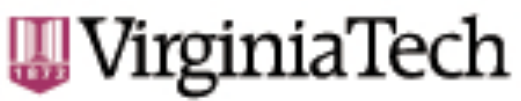

Office of Research Compliance

Institutional Review Board

North End Center, Suite 4120, Virginia Tech

300 Turner Street NW

Blacksburg, Virginia 24061

540/231-4606 Fax 540/231-0959

email irb@vt.edu

website http://www.irb.vt.edu

MEMORANDUM

DATE:

TO:

FROM:

PROTOCOL TITLE:

IRB NUMBER:
October 23, 2015

Manuel A Perez-Quinonez, Yao Wang, Rachel A Diana

Virginia Tech Institutional Review Board (FWA00000572, expires July 29, 2020)

User Study for memorizing location-based to-do tasks

15-676

Effective October 23, 2015, the Virginia Tech Institution Review Board (IRB) Administrator, Carmen T Papenfuss, approved the New Application request for the above-mentioned research protocol.

This approval provides permission to begin the human subject activities outlined in the IRB-approved protocol and supporting documents.

Plans to deviate from the approved protocol and/or supporting documents must be submitted to the IRB as an amendment request and approved by the IRB prior to the implementation of any changes, regardless of how minor, except where necessary to eliminate apparent immediate hazards to the subjects. Report within 5 business days to the IRB any injuries or other unanticipated or adverse events involving risks or harms to human research subjects or others.

All investigators (listed above) are required to comply with the researcher requirements outlined at:

http://www.irb.vt.edu/pages/responsibilities.htm

(Please review responsibilities before the commencement of your research.)

\section{PROTOCOL INFORMATION:}

Approved As:

Protocol Approval Date:

Protocol Expiration Date:

Continuing Review Due Date*:
Expedited, under 45 CFR 46.110 category(ies) 6,7

October 23, 2015

October 22, 2016

October 8, 2016

*Date a Continuing Review application is due to the IRB office if human subject activities covered under this protocol, including data analysis, are to continue beyond the Protocol Expiration Date.

\section{FEDERALLY FUNDED RESEARCH REQUIREMENTS:}

Per federal regulations, 45 CFR 46.103(f), the IRB is required to compare all federally funded grant proposals/work statements to the IRB protocol(s) which cover the human research activities included in the proposal / work statement before funds are released. Note that this requirement does not apply to Exempt and Interim IRB protocols, or grants for which VT is not the primary awardee.

The table on the following page indicates whether grant proposals are related to this IRB protocol, and which of the listed proposals, if any, have been compared to this IRB protocol, if required. 


\begin{tabular}{|l|l|l|l|}
\hline Date* & OSP Number & Sponsor & Grant Comparison Conducted? \\
\hline & & & \\
\hline & & & \\
\hline & & & \\
\hline & & & \\
\hline & & & \\
\hline & & & \\
\hline & & & \\
\hline & & & \\
\hline
\end{tabular}

* Date this proposal number was compared, assessed as not requiring comparison, or comparison information was revised.

If this IRB protocol is to cover any other grant proposals, please contact the IRB office (irbadmin@vt.edu) immediately. 


\section{Virginia Polytechnic and State University Center for Human-Computer Interaction (CHCI) CONSENT FORM}

You are hereby invited to participate in a research study. Please read through the information below carefully and ask any questions that you may have before agreeing to take part in the study.

What the Study is About: The purpose of the study is to understand how people remember and recall the location-based to-do tasks. We are trying to explore the human behaviors of encoding and remembering location-based to-do tasks.

What You will be Asked to Do If you agree to participate in this study, you will be engaged in a two-part of the study, each part will take no more than 30 minutes in the $\mathrm{HCl}$ lab on campus in MCB room 133. The first part of the study will ask you to finish a background questionnaire and memorize some predefined to-do tasks, and the second part of the study will ask you to come back to see how many tasks you can recall and complete a post task questionnaire.

Risks and Benefits: The results of this study will contribute to academic knowledge, and to understanding how people recall to-do tasks and how they use reminders to assist daily remembering. It is not intended to have any direct benefits or risks to you as a participant. Participants will receive SONA credits if they use SONA system to register the study or receive class credits if they are taking CS 3724.

Data Collection: The records of this study will be kept private. A participant ID will be randomly assigned to each participant, and that will be the only thing used during the study and for data analysis. There will be no identifying information attached to the participant ID. Research data will be kept in a secure computer, and only the researchers will have access to the records. The data will be used for publication purposes and for a dissertation.

Audio Recording: With your permission, we will capture the computer screen and audio record your comments. No private information such as name will be stored together with the recordings to identify you. Audio recordings will be permanently deleted after the transcription. The quotes may be used in the publication anonymously.

Taking Part is Voluntary: Your participation in this study is completely voluntary. You may discontinue participation at any time prior to the completion of any part of our study.

If You have any Questions or Inquiries: You may contact Yao Wang at yxw5167@vt.edu/ 585-743-7118 or Dr. Manuel A Perez-Quinones at Perez.Quinones@uncc.edu for questions about the study. You may contact Dr. David Moore at moored@vt.edu or 231-4991 for questions related to your rights as a human subject research participant. 


\section{CONSENT}

You must be 18 years older to be eligible to participate in this study. I certify that I am 18 years old or older. I have read and understand the above information. I have received a copy of this form. I agree to participate in this study.

Subject's signature Date 


\section{Moderator Protocol_for Blacksburg group}

Thank you for your time today. This study aims to help us understand how people remember their to-do tasks.

First of all, I need to make sure you are older than 18 and then sign the consent form.

[Give the consent form]

Now I am going to assign you a participant ID, which is used during the study to fill out the survey questions. Your participant ID is xxx.

\section{Session 1}

This study consists of two sessions, and each session takes no more than 30 minutes. You will need to come back after 3 days to complete the second session.

In the first session of the study, I will present eight location-based tasks you need to remember to do at a later time. In the second session of the study, I will ask you to recall the tasks and locations that you memorized during the first session. You will have to recall the location of where the task needs to be done and the task of what needs to be done.

For both sessions, I will screen capture the computer and audio record your comments. The recordings will be only used for research purposes.

Ready to start?

[Start recording]

We will move forward to the first session now. During the study, feel free to take a rest at any time. Thanks again for your time.

First please complete some background questions.

[Open background questions of BB on Qualtrics]

Now, I am going to give you a list of eight pre-defined to-do tasks, you will enter each task into the computer as if you were adding it to your to-do list; you can type in whatever you would normally do on your real to-do list. At the same time, you will see images representing the business locations you need to visit to complete that task.

Note $^{1}$ : content in the [] is used as a reminder for the moderator to conduct the study. 
Once you have finished entering one task, just click the "next task" button on the computer to move to the next task. After you have finished entering all the eight tasks, click the "I am done" button.

[Give the task scenarios and open the web page for users]

You have finished entering all the to-do tasks. You will have to remember these tasks when you come back in a few days. I would like to confirm the time and date for session 2 .

This is all for the first session of the study. Thank you very much for your time today. I will see you in a couple of days.

\section{Session 2}

Welcome back. Thank you very much for your time. In this session, I will ask you to recall the to-do tasks that you entered into the computer several days ago.

On the screen, you will see a video taken from a car driving through the town of Blacksburg. I would like you to imagine that you are driving the car. As you drive, I want you to count how many traffic lights you come across. Just count the ones you are passing by. I will give you a piece of paper and a pen, so you can use them to count the traffic lights if you want. At the same time, when you see one of the business locations you need to visit, please pause the video and say out loud the task you need to complete at that location.

[Give pen and paper]

First, you can complete a practice session to make sure you understand the task. Image that you need to get a taco from TacoBell. While you are watching the video, you need to count the traffic lights you are passing by and remember to pause the video at where you need to finish this task.

[Play 20 seconds exercise video]

As a reminder, I will screen capture the computer and audio record your comments. The recordings will be only used for research purposes.

[Start recording]

You have a total of eight tasks to identify while you are watching the video. This part of the study is completed when the video comes to the end.

Before we start, do you need some time to think about the to-do tasks?

Note $^{1}$ : content in the [] is used as a reminder for the moderator to conduct the study. 
[Play the video]

Now, I would like you to complete the following questions.

Please rate the following questions based on your general experiences in daily life. [Open familiarity locations of BB on the Qualtrics]

Now I have a couple of quick questions.

1. Have you used or do you use a Location-based reminder system?

If not, explain the concept.

2. How do you think of this type of reminder?

Useful or not? Under what condition might be useful?

3. How do you think the encoding interface helps you remember the location?

4. Do you use any other strategies to remember the location and the task?

5. How do you use GPS differently at your home city vs. when travelling on new cities?

This is the end of the study. Thank you very much for your interests. If you have any further questions and concerns regarding the study, please send me an email at any time.

Note $^{1}$ : content in the [] is used as a reminder for the moderator to conduct the study. 


\section{Moderator Protocol_for San Francisco group}

Thank you for your time today. This study aims to help us understand how people remember their to-do tasks.

First of all, I need to make sure you are older than 18 and then sign the consent form.

[Give the consent form]

Now I am going to assign you a participant ID, which is used during the study to fill out the survey questions. Your participant ID is xxx.

\section{Session 1}

This study consists of two sessions, and each session takes no more than 30 minutes. You will need to come back after 3 days to complete the second session.

In the first session of the study, I will present eight location-based tasks you need to remember to do at a later time. In the second session of the study, I will ask you to recall the tasks and locations that you memorized during the first session. You will have to recall the location of where the task needs to be done and the task of what needs to be done.

For both sessions, I will screen capture the computer and audio record your comments. The recordings will be only used for research purposes.

Ready to start?

[Start recording]

We will move forward to the first session now. During the study, feel free to take a rest at any time. Thanks again for your time.

First please complete some background questions.

[Open background questions on Qualtrics]

Now, I am going to give you a list of eight pre-defined to-do tasks, you will enter each task into the computer as if you were adding it to your to-do list; you can type in whatever you would normally do on your real to-do list. At the same time, you will see images representing the business locations you need to visit to complete that task.

Note $^{1}$ : content in the [] is used as a reminder for the moderator to conduct the study. 
Once you have finished entering one task, just click the "next task" button on the computer to move to the next task. After you have finished entering all the eight tasks, click the "I am done" button.

[Give the task scenarios and open the web page for users]

You have finished entering all the to-do tasks. You will have to remember these tasks when you come back in a few days. I would like to confirm the time and date for session 2 .

This is all for the first session of the study. Thank you very much for your time today. I will see you in a couple of days.

\section{Session 2}

Welcome back. Thank you very much for your time. In this session, I will ask you to recall the to-do tasks that you entered into the computer several days ago.

On the screen, you will see a video taken from a car driving through downtown San Francisco. I would like you to imagine that you are driving the car. As you drive, I want you to count how many traffic lights you come across. Just count the ones you are passing by. I will give you a piece of paper and a pen, so you can use them to count the traffic lights if you want. At the same time, when you see one of the business locations you need to visit, please pause the video and say out loud the task you need to complete at that location.

[Give pen and paper]

First, you can complete a practice session to make sure you understand the task. Image that you need to get a sandwich from Subway. While you are watching the video, you need to count the traffic lights you are passing by and remember to pause the video at where you need to finish this task.

[Play 20 seconds exercise video]

As a reminder, I will screen capture the computer and audio record your comments. The recordings will be only used for research purposes.

[Start recording]

You have a total of eight tasks to identify while you are watching the video. This part of the study is completed when the video comes to the end.

Before we start, do you need some time to think about the to-do tasks?

Note $^{1}$ : content in the [] is used as a reminder for the moderator to conduct the study. 
[Play the video]

Now, I would like you to complete the following questions.

Please rate the following questions based on your general experiences in daily life. [Open familiarity locations of SF on the Qualtrics]

Now I have a couple of quick questions.

1. Have you used or do you use a Location-based reminder system?

If not, explain the concept.

2. How do you think of this type of reminder?

Useful or not? Under what condition might be useful?

3. How do you think the encoding interface helps you remember the location?

4. Do you use any other strategies to remember the location and the task?

5. How do you use GPS differently at your home city vs. when travelling on new cities?

This is the end of the study. Thank you very much for your interests. If you have any further questions and concerns regarding the study, please send me an email at any time.

Note $^{1}$ : content in the [] is used as a reminder for the moderator to conduct the study. 


\section{Session 1 To-do tasks_for Blacksburg group}

Imagine it is $10 \mathrm{am}$ Monday, and you are at school. You want to clean up the tasks that you need to complete during the week, so you start to prepare a to-do list with the tasks listed below. You need to enter all these to-do tasks into the computer.

1. The fall season is coming and you suffer of seasonal allergies. You would like to stop by Target to pick up the allergies prescription drugs on your way home.

Once you have finished memorizing this task, you can click the "next task" button to continue.

2. You plan to go fishing this weekend with several friends. Blacksburg Feed \& Seed Co is the place providing professional fishing supplies. You pass by this store everyday when you are driving home, so you decide to stop by this store to get some fishing supplies.

Once you have finished memorizing this task, you can click the "next task" button to continue.

3. You dropped your suits at the dry cleaners, A Cleaner World, a few days ago. Your laundry is ready to pick up.

Once you have finished memorizing this task, you can click the "next task" button to continue.

4. You live in an apartment, and you need some quarters to use in the washer and dryer for laundry. There is a Wells Fargo bank on your way home, so you want to get some quarters at this bank.

Once you have finished memorizing this task, you can click the "next task" button to continue.

5. Your mom's birthday is coming. Kent Jewelry is offering limited specials. You would like to buy your mom a piece of jewelry for her birthday present.

Once you have finished memorizing this task, you can click the "next task" button to continue.

6. You ordered a wireless printer online, but shipping is expensive. You decided to pick it up at Best Buy store and save the shipping cost. Pick up the printer as you drive by Best Buy. 
Once you have finished memorizing this task, you can click the "next task" button to continue.

7. You would like to mail a package to one of your friends in Canada. Stop by the US Postal Office to drop the package.

Once you have finished memorizing this task, you can click the "next task" button to continue.

8. You received a promotional letter from Carter Bank and Trust for a new credit card. You need to stop by the bank to sign some papers. You decide to do so next time you drive by the bank.

Once you have finished memorizing this task, you can click the "I am done" button to complete the study. 


\section{Session 1 To-do tasks_for San Francisco group}

Imagine it is $10 \mathrm{am}$ on Monday and you are school. You want to clean up the tasks that you need to complete during the week, so you start to prepare a to-do list with the tasks listed below. You need to enter all these to-do tasks into the computer.

1. You ordered a wireless printer online, but shipping is expensive. You decided to ship it to the Office Depot for free. It has arrived, and you want to remember to pick up the printer while you are driving by the Office Depot in downtown.

Once you have finished memorizing this task, you can click the "next task" button to continue.

2. One of your friends borrowed a book from the library at the Academy of Art University. Academy of Art University is near your workplace, so he asked you to return the book for him. You need to remember to return the book when you are passing by the university.

Once you have finished memorizing this task, you can click the "next task" button to continue.

3. Your roommate helped you solve a big problem in your homework yesterday. Peets Coffee and Tea is offering limited holiday specials. As a thank you, you would like to stop by Peets Coffee and Tea to buy a holiday gift for your roommate on the way home.

Once you have finished memorizing this task, you can click the "next task" button to continue.

4. You live in an apartment, and you need some quarters to use the washer and dryer in the basement. There is a Wells Fargo bank near the workplace, so you want to get some quarters when you are near the Wells Fargo.

Once you have finished memorizing this task, you can click the "next task" button to continue.

5. The fall season is coming and you suffer of seasonal allergies. You would like to stop by Walgreens to pick up the allergies prescription drugs after work.

Once you have finished memorizing this task, you can click the "next task" button to continue. 
6. Your mother's birthday is coming. You would like to mail a package to your Mom as a gift. You need to remember to stop by FedEx to drop the package.

Once you have finished memorizing this task, you can click the "next task" button to continue.

7. You received a promotional letter from Citibank. You decided to stop at Citibank to open a new credit card to receive the benefits when you are driving by the bank next time.

Once you have finished memorizing this task, you can click the "next task" button to continue.

8. You are going to host a birthday party in your house this Friday. Target is on your way home; so you decided to stop at Target to buy some party supplies.

Once you have finished memorizing this task, you can click the "I am done" button to complete the study. 


\section{Background Questions for participants in both groups}

1. What is your age?

2. What is your gender?

3. How would you describe your race?
a. White, Caucasian
b. Black, African American
c. American Indian or Alaska Native
d. Asian
e. Native Hawaiian or other Pacific Islander
f. Some other race or origin
g. Prefer not to disclose

4. Are you of Hispanic/Latino/Spanish origin?
a. Yes
b. No
c. Prefer not to disclose

5. What is your dominant language (sometimes known as first language)?

6. What degree are you pursing (bachelors, masters, doctorate?

7. What major are you pursuing?

On a scale of 1-5 to rate how often do the following situations occur to you.

8. Forget an appointment

$\begin{array}{lllll}1 & 2 & 3 & 4 & 5 \\ \text { Never } & \text { Rarely } & \text { Sometimes } & \begin{array}{l}\text { Most of the } \\ \text { time }\end{array} & \text { Always }\end{array}$

9. Forget to pay a bill

$\begin{array}{lllll}1 & 2 & 3 & 4 & 5 \\ \text { Never } & \text { Rarely } & \text { Sometimes } & \begin{array}{l}\text { Most of the } \\ \text { time }\end{array} & \text { Always }\end{array}$


10. Forget to pick up or buy something at a particular place

$\begin{array}{lllll}1 & 2 & 3 & 4 & 5 \\ \text { Never } & \text { Rarely } & \text { Sometimes } & \begin{array}{l}\text { Most of the } \\ \text { time }\end{array} & \text { Always }\end{array}$

11. Forget to bring something with you

$\begin{array}{lllll}1 & 2 & 3 & 4 & 5 \\ \text { Never } & \text { Rarely } & \text { Sometimes } & \begin{array}{l}\text { Most of the } \\ \text { time }\end{array} & \text { Always }\end{array}$

12. Forget to return something or drop something at a particular place

$\begin{array}{lllll}1 & 2 & 3 & 4 & 5 \\ \text { Never } & \text { Rarely } & \text { Sometimes } & \begin{array}{l}\text { Most of the } \\ \text { time }\end{array} & \text { Always }\end{array}$




\section{Post Questions for San Francisco group}

1. How familiar are you with the San Francisco downtown area?

a. Very unfamiliar. Have never been there before

b. Unfamiliar. Have traveled there once or twice

c. Neutral. Have visited several times

d. Familiar. Lived there for a short while, like a couple of months

e. Very familiar. Have lived there for a long time, like a couple of years

2. Have you ever driven a car around downtown San Francisco?

a. Yes

b. No

3. Have you ever taken a tour around downtown San Francisco, either take a bus or walk around?

4. Please circle the location area that you are familiar with on the map showing downtown San Francisco. 
5. Before you completed this experiment, on a scale of 1-5 to rate how familiar are you with the businesses listed below and their logos.

\begin{tabular}{|c|c|c|c|c|c|}
\hline & $\begin{array}{l}\text { Not familiar } \\
\text { at all }\end{array}$ & \multicolumn{2}{|l|}{$\begin{array}{l}\text { Very } \\
\text { familiar }\end{array}$} \\
\hline Office Depot & 1 & 2 & 3 & 4 & 5 \\
\hline $\begin{array}{c}\text { Academy of Art } \\
\text { University }\end{array}$ & 1 & 2 & 3 & 4 & 5 \\
\hline $\begin{array}{c}\text { Peets Coffee and } \\
\text { Tea }\end{array}$ & 1 & 2 & 3 & 4 & 5 \\
\hline \begin{tabular}{c} 
Wells Fargo Bank \\
\hline Walgreens
\end{tabular} & 1 & 2 & 3 & 4 & 5 \\
\hline Fedex & 1 & 2 & 3 & 4 & 5 \\
\hline Citibank & 1 & 2 & 3 & 4 & 5 \\
\hline Target & 1 & 2 & 3 & 4 & 5 \\
\hline
\end{tabular}




\section{Post Questions for Blacksburg group}

1. How familiar are you with the Blacksburg area?

a. Very unfamiliar. Have never been there before

b. Unfamiliar. Have traveled there once or twice

c. Neutral. Have visited several times

d. Familiar. Lived there for a short while, like a couple of months

e. Very familiar. Have lived there for a long time, like a couple of years

2. How long have you lived in the Blacksburg area?

3. In how many different places have you lived in the Blacksburg area?

4. How frequently do you drive a car in the Blacksburg area?
a. Every Day
b. Several times a week
c. Several times a month
d. Only once or twice a month
e. Never drive a car

5. How frequently do you take a bus to school?
a. Every Day
b. Several times a week
c. Several times a month
d. Only once or twice a month
e. Never take a bus to school

6. If you ever took a bus to school, which bus line did you take?

7. Please circle the location area that you are familiar with on the map showing the Blacksburg.

8. Before you completed this experiment, on a scale of 1-5 to rate how familiar are you with the businesses listed below and their logos 


\begin{tabular}{|c|c|c|c|c|c|}
\hline & $\begin{array}{l}\text { Not familiar } \\
\text { at all }\end{array}$ & \multicolumn{2}{|l|}{ Very familiar } \\
\hline Target & 1 & 2 & 3 & 4 & 5 \\
\hline $\begin{array}{c}\text { Blacksburg Feed } \\
\text { and Seed Co }\end{array}$ & 1 & 2 & 3 & 4 & 5 \\
\hline A Cleaner World & 1 & 2 & 3 & 4 & 5 \\
\hline $\begin{array}{c}\text { Wells Fargo Bank } \\
\text { Kent Jewelry }\end{array}$ & 1 & 2 & 3 & 4 & 5 \\
\hline $\begin{array}{c}\text { Best Buy } \\
\text { USPS }\end{array}$ & 1 & 2 & 3 & 4 & 5 \\
\hline Carter Bank and & 1 & 2 & 3 & 4 & 5 \\
\hline Trust & 1 & 2 & 3 & 4 & 5 \\
\hline
\end{tabular}




\section{Post Test Interview Questions for both groups}

1. Have you used or do you use a Location-based reminder system?

If not, explain the concept.

2. How do you think of this type of reminder?

Useful or not? Under what condition might be useful?

3. How do you think the encoding interface helps you remember the location?

4. Do you use any other strategies to remember the location and the task?

5. How do you use GPS differently at your home city vs. when travelling on new cities? 
Appendix D

Prototype Evaluation Materials for

Chapter 9 


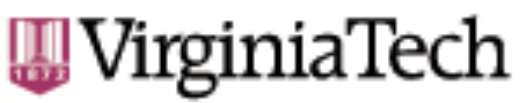

Office of Research Compliance

Institutional Review Board

North End Center, Suite 4120, Virginia Tech

300 Turner Street NW

Blacksburg, Virginia 24061

540/231-4606 Fax 540/231-0959

email irb@vt.edu

website http://www.irb.vt.edu

MEMORANDUM

DATE:

TO:

FROM:
March 20, 2017

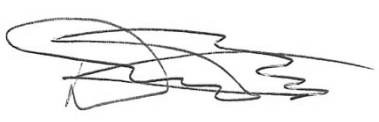

Rachel A Diana, Yao Wang, Manuel A Perez-Quinonez

Virginia Tech Institutional Review Board (FWA00000572, expires January 29, 2021)

PROTOCOL TITLE: Location-based reminder prototype evaluation

IRB NUMBER:

\section{7-195}

Effective March 17, 2017, the Virginia Tech Institution Review Board (IRB) Chair, David M Moore, approved the New Application request for the above-mentioned research protocol.

This approval provides permission to begin the human subject activities outlined in the IRB-approved protocol and supporting documents.

Plans to deviate from the approved protocol and/or supporting documents must be submitted to the IRB as an amendment request and approved by the IRB prior to the implementation of any changes, regardless of how minor, except where necessary to eliminate apparent immediate hazards to the subjects. Report within 5 business days to the IRB any injuries or other unanticipated or adverse events involving risks or harms to human research subjects or others.

All investigators (listed above) are required to comply with the researcher requirements outlined at: http://www.irb.vt.edu/pages/responsibilities.htm

(Please review responsibilities before the commencement of your research.)

\section{PROTOCOL INFORMATION:}

Approved As:

Protocol Approval Date:

Protocol Expiration Date:

Continuing Review Due Date*:
Expedited, under 45 CFR 46.110 category(ies) 6,7

March 17, 2017

March 16, 2018

March 2, 2018

*Date a Continuing Review application is due to the IRB office if human subject activities covered under this protocol, including data analysis, are to continue beyond the Protocol Expiration Date.

\section{FEDERALLY FUNDED RESEARCH REQUIREMENTS:}

Per federal regulations, 45 CFR 46.103(f), the IRB is required to compare all federally funded grant proposals/work statements to the IRB protocol(s) which cover the human research activities included in the proposal / work statement before funds are released. Note that this requirement does not apply to Exempt and Interim IRB protocols, or grants for which VT is not the primary awardee.

The table on the following page indicates whether grant proposals are related to this IRB protocol, and which of the listed proposals, if any, have been compared to this IRB protocol, if required.

\section{Invent the Future}

VIRGINIA POLYTECHNIC INSTITUTE AND STATE UNIVERSITY An equal opportunity, affirmative action institution 


\begin{tabular}{|l|l|l|l|}
\hline Date* & OSP Number & Sponsor & Grant Comparison Conducted? \\
\hline & & & \\
\hline & & & \\
\hline & & & \\
\hline & & & \\
\hline & & & \\
\hline & & & \\
\hline & & & \\
\hline & & & \\
\hline
\end{tabular}

* Date this proposal number was compared, assessed as not requiring comparison, or comparison information was revised.

If this IRB protocol is to cover any other grant proposals, please contact the IRB office (irbadmin@vt. edu) immediately. 


\section{Virginia Polytechnic and State University Center for Human-Computer Interaction ( $\mathrm{CHCl}$ ) CONSENT FORM}

You are hereby invited to participate in a research study. Please read through the information below carefully and ask any questions that you may have before agreeing to take part in the study.

What the Study is About: The purpose of the study is to understand how people use a location-based reminder system. We are trying to uncover usability issues and understand users' satisfaction about the design of a location-based reminder prototype. The results will be used for dissertation and might be published.

What You will be Asked to Do If you agree to participate in this study, you will be engaged in a 1-hour study in the $\mathrm{HCl}$ lab on campus in MCB room 133. You will be asked to use a given location-based reminder prototype on a given IPhone to set up three location-based reminders. Subjective comments will be collected while using the reminder. A short one-on-one interview will be conducted at the end of the study to understand your overall using experiences.

Risks and Benefits: The results of this study will contribute to academic knowledge, and to provide design implications for future location-based reminder systems. It is not intended to have any direct benefits or risks to you as a participant. Participants will receive SONA credits if they use SONA system to register the study.

Data Collection: The records of this study will be kept private. A participant ID will be randomly assigned to each participant, and that will be the only thing used during the study and for data analysis. There will be no identifying information attached to the participant ID. Research data will be kept in a secure computer, and only the researchers will have access to the records. The data will be used for publication purposes and for a dissertation.

Audio Recording: With your permission, we will capture the computer screen and audio record your comments. No private information such as name will be stored together with the recordings to identify you. Audio recordings will be permanently deleted after the transcription. The quotes may be used in the publication anonymously.

Taking Part is Voluntary: Your participation in this study is completely voluntary. You may discontinue participation at any time prior to the completion of any part of our study.

If You have any Questions or Inquiries: You may contact Yao Wang at yxw5167@vt.edu / 585-743-7118 or Dr. Rachel Diana at rdiana@vt.edu for questions about the study. You may contact Dr. David Moore at moored@vt.edu or 231-4991 for questions related to your rights as a human subject research participant. 


\section{CONSENT}

You must be 18 years older to be eligible to participate in this study. I certify that I am 18 years old or older. I have read and understand the above information. I have received a copy of this form. I agree to participate in this study.

Subject's signature Date 


\section{Moderator Protocol}

\section{Orientation Script}

Thank you very much for your interest to take part in my research study. My name is Yao, I am here to learn how users interact with a location-based reminder system. This study aims to understand and improve the users' satisfaction of using a location-based mobile reminder prototype. During the test session, I will ask you to use the mobile phone, which I will provide to you later, to set up a list of location-based reminders. As you work on these tasks, please try to do whatever you would normally do. I will make a direct observation when you are doing the tasks.

Please know that we are not testing you, and there is no such thing as a wrong answer. Your doing this helps me understand what works or doesn't work about this mobile reminder system. This test doesn't examine you or your ability but rather how easy to use this mobile reminder system.

Please try to think out loud while you are working. Just tell me whatever is going through your mind when you are performing the tasks. Simply express your thoughts about what you are looking for to click and why.

Your participation is completely voluntary and you may discontinue your participation at any time. The whole session will take about 1 hour. You are welcome to take a break at any time. Please let me know if you would like a break.

Thank you again for your participation. Do you have any questions before we start?

\section{Task Scenario}

Task1: Your roommate just texted you a message and asked you to pick up some milk from the grocery store when you are driving home. To complete the task, you decide to create a reminder for picking up milk, so it can be delivered when you are near a grocery store.

Task2: One of your friends is studying at Academy of Art University at San Francisco. You are invited to visit her during the Spring break. You want to pick up a piece of new art design in the school library while leaving, so you set up a reminder to remind you to pick up the design product while you are near the school library.

Task3: You are driving to Charlotte to visit a family member. You decide to order a pizza from Mellow Mushroom for dinner. You set up a reminder for picking it up while on the way near the Mellow Mushroom in Downtown area. 


\section{Post-test Questionnaire}

1. Do you find any difficulties using this interface to set up the reminder?

2. How are you satisfied with your using experience?

3. How do you like/dislike the reminder system?

4. How easy was it for you to set up the reminder using a Street View?

5. Do you have any suggestions of the interface design? 